\title{
Quantum Computing with Rotation-Symmetric Bosonic Codes
}

\author{
Arne L. Grimsmo®, ${ }^{1, *}$ Joshua Combes $\odot,{ }^{2}$ and Ben Q. Baragiola $\odot^{3}$ \\ ${ }^{1}$ Centre for Engineered Quantum Systems, School of Physics, The University of Sydney, Sydney, Australia \\ ${ }^{2}$ Centre for Engineered Quantum Systems, School of Mathematics and Physics, \\ The University of Queensland, St. Lucia, Queensland 4072, Australia \\ ${ }^{3}$ Centre for Quantum Computation and Communication Technology, School of Science, \\ RMIT University, Melbourne, Victoria 3001, Australia
}

(Received 31 January 2019; revised manuscript received 3 September 2019; accepted 23 December 2019; published 6 March 2020)

\begin{abstract}
Bosonic rotation codes, introduced here, are a broad class of bosonic error-correcting codes based on phase-space rotation symmetry. We present a universal quantum computing scheme applicable to a subset of this class-number-phase codes - which includes the well-known cat and binomial codes, among many others. The entangling gate in our scheme is code agnostic and can be used to interface different rotationsymmetric encodings. In addition to a universal set of operations, we propose a teleportation-based errorcorrection scheme that allows recoveries to be tracked entirely in software. Focusing on cat and binomial codes as examples, we compute average gate fidelities for error correction under simultaneous loss and dephasing noise and show numerically that the error-correction scheme is close to optimal for error-free ancillae and ideal measurements. Finally, we present a scheme for fault-tolerant, universal quantum computing based on the concatenation of number-phase codes and Bacon-Shor subsystem codes.
\end{abstract}

DOI: $10.1103 /$ PhysRevX.10.011058

Subject Areas: Quantum Physics, Quantum Information

\section{INTRODUCTION}

Encoding quantum information into bosonic systems [1-3] is an alternative to conventional error-correcting codes based on discrete two- (or few-) state systems. The infinite Hilbert space of a single bosonic mode provides a playground for redundant digital encodings that can be tailored to a specific task [4-10]. A natural application is to use a bosonic code at the ground level in a concatenated error-correction scheme to suppress errors below the fault-tolerance threshold of a conventional qubit-based code $[3,11]$, potentially reducing the total overhead. Decoders that exploit the continuous-variable nature of bosonic codes can improve the fault-tolerance threshold [12-14] and reduce the number of physical qubits required [15]. From a hardware perspective, wellcontrolled, low-loss bosonic modes occur in many quantum technology platforms, such as electromagnetic modes in optical cavities [16,17] and free space [18], superconducting circuits and microwave cavities [19-23], and motional modes in ion traps [24-26].

\footnotetext{
*arne.grimsmo@sydney.edu.au
}

Published by the American Physical Society under the terms of the Creative Commons Attribution 4.0 International license. Further distribution of this work must maintain attribution to the author(s) and the published article's title, journal citation, and DOI.
Gottesman, Kitaev, and Preskill (GKP) introduced a seminal scheme for quantum computing with bosonic codes, based on states with discrete translation symmetry in phase space [3]. While GKP code words were recently prepared and manipulated in the laboratory [26], experimental progress with other bosonic codes-especially the cat $[2,5,27,28]$ and binomial code families [6] - is more advanced. In a breakthrough experiment, Ofek et al. [29] demonstrated enhancement in the lifetime of a cat-code qubit compared to an unencoded qubit using the same hardware-the so-called break-even point for error correction. This experiment was the first error-correction scheme to achieve this milestone in an experiment. A similar experiment using binomial codes also came very close to break even [30]. Initial steps toward fault-tolerant error correction with these codes have been made recently [31].

In this work, we show how cat and binomial codes belong to a larger family of bosonic codes characterized by discrete rotation symmetry in phase space, in analogy to the discrete translation symmetry of GKP codes. Specifically, we consider codes where a single qubit is encoded into a subspace in which the discrete rotation operator $\hat{Z}_{N}=$ $\exp [i(\pi / N) \hat{n}]$ acts as logical $\bar{Z}$, where $\hat{n}$ is the Fock-space number operator. We refer to these codes as bosonic rotation codes. The parameter $N$ here quantifies the degree of discrete rotation symmetry for the code: It immediately follows that the operator $\hat{R}_{N}=\hat{Z}_{N}^{2}$ acts as the identity on 
the code space; i.e., any state in the code space is $N$-fold rotation symmetric in the sense that it is invariant under a rotation by $2 \pi / N$.

A consequence of $N$-fold rotation symmetry is that an encoded state $\left|\psi_{N}\right\rangle$ has support only on every $N$ th Fock state, $\left|\psi_{N}\right\rangle=\sum_{k=0}^{\infty} c_{k N}|k N\rangle$. The degree of rotation symmetry $N$ thus quantifies the magnitude of a detectable shift in number and sets a code distance in Fock space. A complementary notion of distance for phase-space rotations, $\pi / N$, quantifies detectable rotation errors and reveals a trade-off between detectable number-shift and rotation errors.

A special subset of the bosonic rotation codes we introduce are number-phase codes, which are rotated superpositions of states with a small phase uncertainty [32]. We show that a vanishing phase uncertainty is related to (approximate) number-translation symmetry. In the limit of vanishing phase uncertainty, the number-phase codes are analogous to ideal GKP codes, with number and phase playing dual roles in place of position and momentum. Interestingly, we show that cat codes, binomial codes, and the shift-resistant qudit codes introduced in Ref. [3] each approach this ideal limit of vanishing phase uncertainty as their average excitation number increases.

We present a scheme for universal quantum computation for number-phase codes, where the workhorse is an entangling controlled-rotation (СROT) gate based on a cross-Kerr interaction $(\sim \hat{n} \otimes \hat{n})$. A properly tuned CROT serves as a logical controlled- $Z, \hat{C}_{Z}=\operatorname{diag}(1,1,1,-1)$, between two number-phase codes. Notably, codes with different degrees of rotation symmetry and of different type (e.g., cat and binomial) can be fully entangled by the CROT gate. Similarly, a self-Kerr interaction $\left(\sim \hat{n}^{2}\right)$ can be used to enact the single-qubit phase gate $\hat{S}=\operatorname{diag}(1, i)$. Augmented with preparation of dual-basis code words $\left|+_{N}\right\rangle$ and destructive measurements in the dual basis, all Clifford gates can be executed. Universality is achieved using injected magic states. The gates we introduce have favorable properties in terms of fault tolerance: Errors are amplified and spread in a very limited way, such that the "size" of an error after a gate is proportional to the "size" prior to the gate.

We also introduce a new error-correction scheme for the number-phase codes discussed above. The error correction is based on teleporting the encoded information into a new, fresh ancilla [33]. A remarkable feature of this approach is that recovery operations can be tracked entirely in software. In essence, the need for potentially difficult and highly nonlinear operations to restore the code space is replaced by preparation of ancillae in logical basis states.

We perform a numerical study of error-correction performance in the presence of simultaneous loss and dephasing noise on encoded qubits, while assuming noiseless ancillae. A critical question for a bosonic error-correcting code is whether it performs better than the "trivial encoding" - a qubit encoded in Fock states $|0\rangle$ and $|1\rangle$ under the same noise model. The point where the errorcorrected qubit performs as well as the trivial encoding is referred to as the break-even point for error correction. For the studied noise model, we find that both cat and binomial codes go beyond break even by several orders of magnitude for degrees of rotation symmetry in the range $N=2-4$ and dimensionless noise strength for simultaneous loss and dephasing in the range $\kappa t=10^{-3}-10^{-2}$. Remarkably, we also find that the teleportation-based error-correction scheme we introduce performs nearly as well as the optimal recovery map (found numerically), for noise-free ancillae and idealized measurements.

Finally, we outline a fault-tolerant and universal scheme based on concatenating number-phase codes with BaconShor subsystem codes [34-37]. The concatenation serves three purposes: to deal with errors in state preparation which are hard to check for, to suppress errors in noisy measurements by exploiting correlations across multiple measurement outcomes, and to further lower the logical error rate by dealing with errors that are too large for the bosonic code to handle. Our specific example using a Bacon-Shor code illustrates the broader point that a faulttolerant scheme should be tailored to the strengths and weaknesses of the bosonic code at the ground level.

This paper is laid out as follows. In Sec. II, we detail the structure of bosonic rotation codes and describe how to distinguish the code words in the computational and dual bases using number and phase measurements, respectively. We introduce a measure of modular phase uncertainty that quantifies the embedded phase-measurement error in a given bosonic rotation code. In Sec. III, we define number-phase codes, a family of bosonic rotation codes with small modular phase uncertainty, and we give examples of number-phase codes, including cat and binomial codes. In Sec. IV, we present a universal scheme for quantum computing with number-phase codes. In Sec. V, we give a method to perform a modular excitation number measurement, $\hat{n} \bmod N$, which can be used to detect number errors and to prepare bosonic rotation code words. In Sec. VI, we study in detail error propagation and show that the gates in our set map small errors to small errors and are in that sense fault tolerant. In Sec. VII, we lay out a scheme for teleportation-based error correction that can be used for any number-phase code, and we numerically test the performance of our error-correction procedure for cat and binomial codes under simultaneous loss and dephasing. In Sec. VIII, we outline a fault-tolerant scheme for quantum computing based on the concatenation of number-phase codes and Bacon-Shor subsystem codes. Finally, in Sec. IX, we summarize our results and highlight open problems.

\section{BOSONIC ROTATION CODES}

Single-mode bosonic codes utilize the large Hilbert space of a bosonic mode to protect logical quantum 

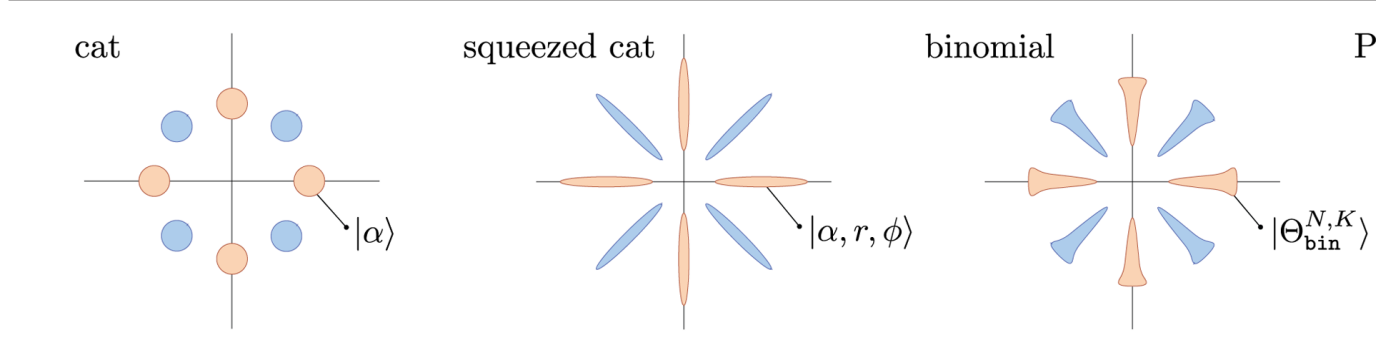

Pegg-Barnett
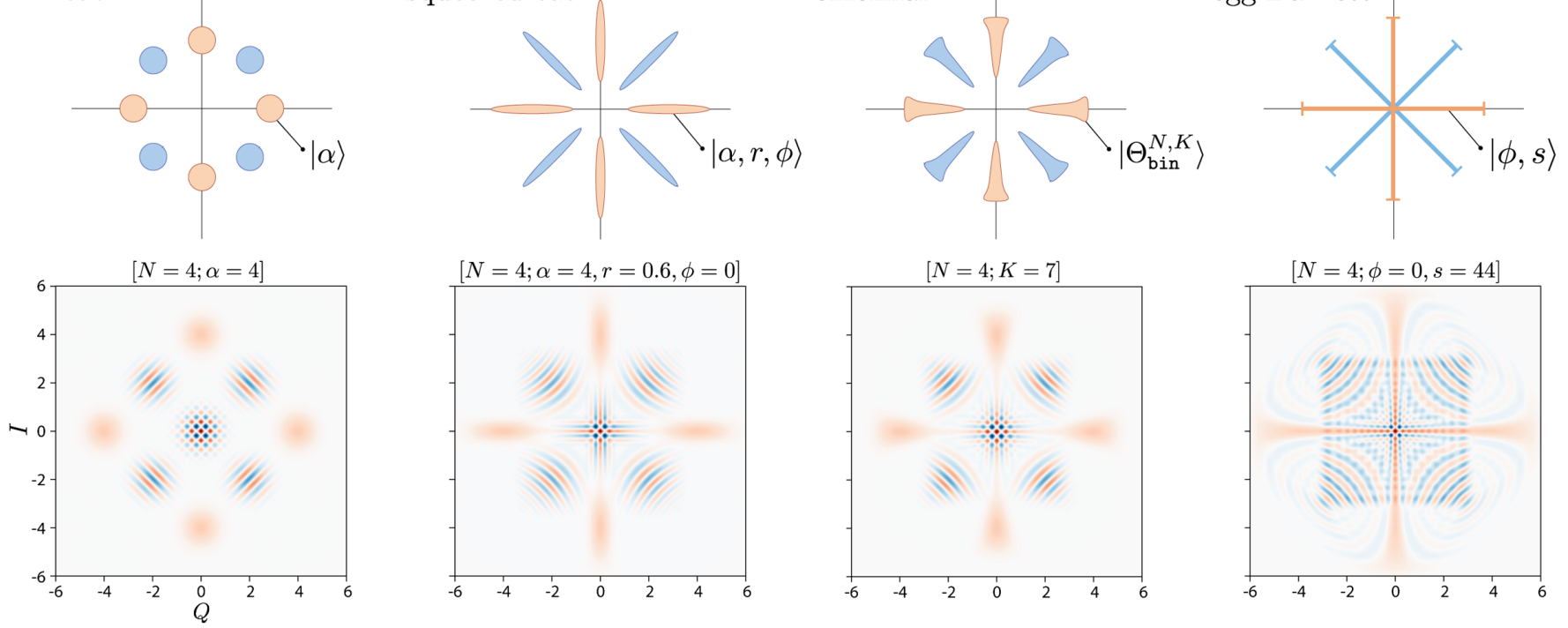

FIG. 1. Graphical summary of several $N=4$ rotation codes: cat and squeezed cat (Appendix B 1), binomial (Appendix B 2), and Pegg-Barnett (Appendix B 3). Logical code words are +1 eigenstates of the discrete rotation operator [Eq. (1)] and exhibit $N$-fold rotation symmetry. Top row: Ball-and-stick diagrams illustrating $\left|+_{N}\right\rangle$ (orange) and $\left|-_{N}\right\rangle$ (blue). Indicated on each is the primitive $|\Theta\rangle$ for the code. Bottom row: Wigner functions for the $\left|+_{N}\right\rangle$ state $W_{\left|+_{N}\right\rangle}(\alpha)$. Red (blue) is positive (negative), the color scale on each plot is different, and $Q=\frac{1}{2}\left(\alpha+\alpha^{*}\right)$ and $I=(1 / 2 i)\left(\alpha-\alpha^{*}\right)$ are the real and imaginary parts, respectively, of $\alpha$.

information. There are countless ways to embed a qubit, with each encoding comprising a two-dimensional subspace spanned by states serving as the logical code words and the remaining Hilbert space providing the freedom to detect and correct errors. In many physical implementations, the dominant sources of noise are loss, gain, and dephasing. Various bosonic qubit encodings have been designed specifically to counteract these noise processes, notably cat $[2,5,27,28]$ and binomial codes [6], which share the key property that the code words exhibit discrete rotational symmetry.

We use discrete rotation symmetry to define a class of single-mode codes called bosonic rotation codes. First, we say that a code has discrete $N$-fold rotation symmetry if any state $|\psi\rangle$ in the code subspace (code space) is a +1 eigenstate of the discrete rotation operator [38]

$$
\hat{R}_{N}:=e^{i(2 \pi / N) \hat{n}},
$$

where $\hat{n}=\hat{a}^{\dagger} \hat{a}$ is the Fock-space number operator $\left(\left[\hat{a}, \hat{a}^{\dagger}\right]=1\right)$. It follows that the square root of $\hat{R}_{N}$ preserves the rotation symmetry and acts as a Hermitian operator that squares to the identity on the code space. We define an order- $N$ bosonic rotation code, or rotation code for short, to be a code where the operator

$$
\hat{Z}_{N}:=\hat{R}_{2 N}=e^{i(\pi / N) \hat{n}}
$$

acts as logical $\bar{Z}$. The choice of $\bar{Z}$ over $\bar{X}$ is just a convention, but we emphasize that a code where $\hat{Z}_{N}$ acts as a non-Pauli operator is not a rotation code according to our definition. An example of the latter is a square-lattice GKP code where $\bar{Z}_{2}$ acts as a logical Hadamard. This distinction is important when discussing "easy" and "hard" gates for different codes. We briefly comment on the rotation symmetry of GKP codes in the Appendix C.

The logical code words for any order- $N$ rotation code can be constructed from discrete rotated superpositions of a normalized primitive state $|\Theta\rangle$. In fact, we have that two states $\left|0_{N, \Theta}\right\rangle$ and $\left|1_{N, \Theta}\right\rangle$ satisfy $\hat{Z}_{N}\left|j_{N, \Theta}\right\rangle=(-1)^{j}\left|j_{N, \Theta}\right\rangle$ if and only if the two states can be written in the following form:

$$
\begin{gathered}
\left|0_{N, \Theta}\right\rangle:=\frac{1}{\sqrt{\mathcal{N}_{0}}} \sum_{m=0}^{2 N-1} e^{i(m \pi / N) \hat{n}}|\Theta\rangle, \\
\left|1_{N, \Theta}\right\rangle:=\frac{1}{\sqrt{\mathcal{N}_{1}}} \sum_{m=0}^{2 N-1}(-1)^{m} e^{i(m \pi / N) \hat{n}}|\Theta\rangle,
\end{gathered}
$$

where $\mathcal{N}_{i}$ are normalization constants. There is a technical constraint on $|\Theta\rangle$ for the code words to be nonzero, which we specify precisely in Sec. II A. When the rotated primitives appearing in Eq. (3) are orthogonal, $\left\langle\Theta\left|\left(\hat{Z}_{N}\right)^{m}\right| \Theta\right\rangle=0$ for $0<m<2 N$, then $\mathcal{N}_{0}=\mathcal{N}_{1}=2 N$. Generally, however, they are not orthogonal, and the normalization constants are different, $\mathcal{N}_{0} \neq \mathcal{N}_{1}$. Regardless, the code words themselves are exactly orthogonal for any valid $|\Theta\rangle$, and any state in the code space, $\left|\psi_{N}\right\rangle=a\left|0_{N}\right\rangle+b\left|1_{N}\right\rangle$, has $N$-fold rotation symmetry, $\hat{R}_{N}\left|\psi_{N}\right\rangle=\left|\psi_{N}\right\rangle$. Here and henceforth, we suppress the $\Theta$ subscript when referring to a generic rotation code of order $N$ unless it is required for clarity. 
From the above, we recognize that there are many different rotation codes with the same degree of rotation symmetry $N$. While a given primitive $|\Theta\rangle$ uniquely defines an order- $N$ rotation code, there are many possible primitives that give rise to the same code. Several examples of rotation codes are shown in Fig. 1. The best known example is cat codes, where the primitive is a coherent state $\left|\Theta_{\text {cat }}\right\rangle=|\alpha\rangle[5,39]$. Another simple example is the "ON codes" $\left|0_{\text {ON }}\right\rangle:=|0\rangle,\left|1_{\text {ON }}\right\rangle:=|N\rangle[40,41]$, which can be constructed, e.g., from $\left|\Theta_{0 N}\right\rangle=(|0\rangle+|N\rangle) / \sqrt{2}$.

The form of Eq. (3) is reminiscent of GKP codes whose code words are superpositions of translated squeezed states [3]. However, due to the natural $2 \pi$ periodicity of rotations, only a finite number of superpositions are required, and the resulting code word always remains physical, because the rotation operator in Eq. (2) conserves the excitation number, unlike the displacement operator used to define the GKP code words. In Sec. III, we make a tighter analogy between GKP codes and a subclass of rotation codes called number-phase codes.

\section{A. Fock-space structure}

Discrete rotational symmetry enforces a spacing of the code words in Fock space, which underpins (but does not guarantee) the codes' robustness to loss and gain errors. The link between rotation symmetry and spacing in Fock space can be seen by acting the operator $\hat{Z}_{N}$ on an arbitrary state $|\psi\rangle=\sum_{n} a_{n}|n\rangle: \hat{Z}_{N}|\psi\rangle=\sum_{n} e^{i \pi n / N} a_{n}|n\rangle$. Clearly, $|\psi\rangle$ is a +1 eigenstate of $\hat{Z}_{N}$ if and only if $a_{n}=0$ for all $n \neq 2 k N$ for integer $k$. Similarly, $|\psi\rangle$ is a -1 eigenstate if and only if $a_{n}=0$ for all $n \neq(2 k+1) N$. This result leads to the following general form for any \pm 1 eigenstates of $\hat{Z}_{N}$ :

$$
\begin{gathered}
\left|0_{N}\right\rangle=\sum_{k=0}^{\infty} f_{2 k N}|2 k N\rangle, \\
\left|1_{N}\right\rangle=\sum_{k=0}^{\infty} f_{(2 k+1) N}|(2 k+1) N\rangle .
\end{gathered}
$$

The coefficients $f_{k N}$ in Eq. (4) are related to the Fock-state coefficients of the primitive $|\Theta\rangle=\sum_{n} c_{n}|n\rangle$ in Eq. (3) as follows: $f_{2 k N}=c_{2 k N} / \sqrt{\mathcal{N}_{0}^{*}}$ and $f_{(2 k+1) N}=c_{(2 k+1) N} / \sqrt{\mathcal{N}_{1}^{*}}$, where $\mathcal{N}_{0}^{*}=\sum_{k}\left|c_{2 k N}\right|^{2}$ and $\mathcal{N}_{1}^{*}=\sum_{k}\left|c_{(2 k+1) N}\right|^{2}$. The normalization factors introduced here are related to the normalization constants in Eq. (3) as $\mathcal{N}_{i}^{*}=\mathcal{N}_{i} /(2 N)^{2}$. There are no further restrictions on the coefficients $f_{k N}$ apart from normalization.

To explicitly connect the two representations of rotationsymmetric code words [Eqs. (3) and (4)], we use the relation for a Kronecker comb,

$$
\frac{1}{M} \sum_{m=0}^{M-1} e^{i(2 \pi m n / M)}=\sum_{k=0}^{\infty} \delta_{n, k M} \quad \text { for } n=0,1,2, \ldots
$$

to construct a projector onto the set of Fock states $|2 k N+\ell\rangle$ for $k=0,1,2 \ldots[42]$ :

$$
\begin{aligned}
\hat{\Pi}_{2 N}^{\ell} & :=\sum_{k=0}^{\infty}|2 k N+\ell\rangle\langle 2 k N+\ell| \\
& =\frac{1}{2 N} \sum_{m=0}^{2 N-1}\left(e^{-i(\pi \ell / N)} \hat{Z}_{N}\right)^{m} .
\end{aligned}
$$

The factors $e^{i \pi \ell / N}$ with $\ell \in\{0,1, \ldots, 2 N-1\}$ are the complex $2 N$ th roots of unity. Acting the projector on a primitive $|\Theta\rangle=\sum_{n} c_{n}|n\rangle$ with at least one nonzero $c_{2 k N+\ell}$,

$$
\hat{\Pi}_{2 N}^{\ell}|\Theta\rangle=\sum_{k=0}^{\infty} c_{2 k N+\ell}|2 k N+\ell\rangle,
$$

produces an (unnormalized) eigenstate of the operator $\hat{Z}_{N}$ with eigenvalue $e^{i \pi \ell / N}$ [43].

It is now straightforward to see that Eqs. (3) and (4) are equivalent, since we can write

$$
\begin{aligned}
\left|0_{N}\right\rangle & =\frac{\hat{\Pi}_{2 N}^{0}|\Theta\rangle}{\sqrt{\mathcal{N}_{0}^{*}}}, \\
\left|1_{N}\right\rangle & =\frac{\hat{\Pi}_{2 N}^{N}|\Theta\rangle}{\sqrt{\mathcal{N}_{1}^{*}}},
\end{aligned}
$$

with $\mathcal{N}_{0}^{*}=\left\langle\Theta\left|\hat{\Pi}_{2 N}^{0}\right| \Theta\right\rangle$ and $\mathcal{N}_{1}^{*}=\left\langle\Theta\left|\hat{\Pi}_{2 N}^{N}\right| \Theta\right\rangle$. With the help of Eq. (6), we recognize these as Eq. (3a) and (3b). For the code words to be well defined, it is now clear that the primitive must have support on at least one of the $|2 k N\rangle$ Fock states and one of the $|(2 k+1) N\rangle$ Fock states. This requirement is the only constraint on $|\Theta\rangle$, and the orthogonality of the code words is then easily seen from the fact that they have support on strictly different Fock states.

The dual-basis code words $\left| \pm_{N}\right\rangle$ are constructed as usual via superpositions of the computational basis code words $\left| \pm_{N}\right\rangle=\left(\left|0_{N}\right\rangle \pm\left|1_{N}\right\rangle\right) / \sqrt{2}$ leading to Fock-space representations

$$
\begin{gathered}
\left|+_{N}\right\rangle=\frac{1}{\sqrt{2}} \sum_{k=0}^{\infty} f_{k N}|k N\rangle, \\
\left|-{ }_{N}\right\rangle=\frac{1}{\sqrt{2}} \sum_{k=0}^{\infty}(-1)^{k} f_{k N}|k N\rangle .
\end{gathered}
$$

Both $\left| \pm_{N}\right\rangle$ have support on the full set of $|k N\rangle$ Fock states. From Eq. (4a) and (9a), it is clear that any logical $\left|0_{N}\right\rangle$ state is also a logical $\left|+_{2 N}\right\rangle$ for a code with twice the rotation symmetry. In other words, for a given code word $\left|0_{N, \Theta}\right\rangle$ defined by a primitive $|\Theta\rangle$, we have that

$$
\left|0_{N, \Theta}\right\rangle=\left|+_{2 N, \Theta^{\prime}}\right\rangle,
$$


for some primitive $\left|\Theta^{\prime}\right\rangle$. Whenever the code word normalization constant in Eq. (3) satisfies $\mathcal{N}_{0}=\mathcal{N}_{1}$, the above holds with $|\Theta\rangle=\left|\Theta^{\prime}\right\rangle$. See Appendix A for further details.

While it is evident that $\hat{Z}_{N}\left|j_{N}\right\rangle=(-1)^{j}\left|j_{N}\right\rangle$ for $j=0,1$, it is not generally possible to find a similarly simple operator that acts as a logical $\bar{X}$ within the code space. In Sec. III, we present a set of rotation codes whose logical $\bar{X}$ is approximately given by a number-translation operator. The absence of a simple logical $\bar{X}$ does not fundamentally pose a problem for quantum computing, since universality can be achieved without this operator as shown in Sec. IV.

A useful quantity for comparing codes is the average excitation number of the code,

$$
\bar{n}_{\mathrm{Code}}:=\frac{1}{2} \operatorname{tr}\left[\hat{\Pi}_{\mathrm{code}} \hat{n}\right]
$$

where $\hat{\Pi}_{\text {code }}:=\left|0_{N}\right\rangle\left\langle 0_{N}|+| 1_{N}\right\rangle\left\langle 1_{N}\right|$ is a two-dimensional projector onto the code space $[44,45]$. By construction, the average excitation number is the same for each of the dualbasis code words, $\bar{n}_{\text {code }}=\left\langle \pm_{N}|\hat{n}| \pm_{N}\right\rangle$. Since the rotation operator commutes with the number operator, $\left[\hat{R}_{N}, \hat{n}\right]=0$, rotations do not increase the excitation number. The computational-basis code words have different average excitation numbers due to different phases in the superpositions in Eq. (3), but it remains bounded. This result is in contrast to translation-based GKP codes that suffer from the following: Either the code states are unphysical as they have infinite excitations, or the discrete translational symmetry is spoiled by energy constraints.

The $O \mathrm{~N}$ codes introduced previously are the order- $N$ rotation codes with the lowest possible mean excitation number $\bar{n}_{0 \mathrm{~N}}=(N / 2)$. It is worth noting that the trivial encoding where a qubit is encoded in the two lowest Fock states,

$$
\left|0_{\text {triv }}\right\rangle:=|0\rangle, \quad\left|1_{\text {triv }}\right\rangle:=|1\rangle,
$$

is a rotation code with $N=1$. The trivial encoding serves as a useful benchmark against which other codes can be compared $[29,30,44]$.

\section{B. The phase and Fock grids}

With a given primitive $|\Theta\rangle$, the order of rotation symmetry $N$ parameterizes a code both in phase space and in Fock space, via Eq. (3) and (4). From these two forms of the code words, it is natural to define a number distance and rotational distance of a code as [46]

$$
d_{n}:=N, \quad d_{\theta}:=\pi / N .
$$

respectively. Number and phase-space rotation play dual roles: Codes of increasing $N$ are further separated in Fock space but more closely separated in terms of rotations. For an order- $N$ rotation code, we refer to the set of angles

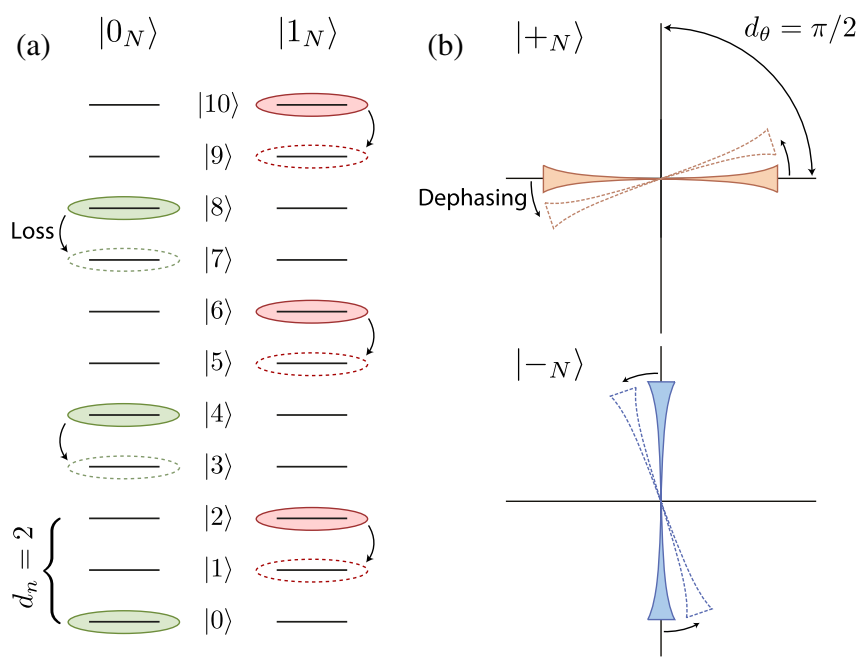

FIG. 2. Graphical summary of the Fock-space and phase-space structure of code words for an $N=2$ rotation code. (a) The computational-basis code words $\left|0_{N}\right\rangle$ and $\left|1_{N}\right\rangle$ have support on every $2 k N$ and $(2 k+1) N$ Fock state for $k=0,1,2, \ldots$, respectively. Up to $N-1$, loss or gain errors can, in principle, be detected. (b) The dual code words $\left| \pm_{N}\right\rangle$ are related by a rotation in phase space by $\pi / 2$. Rotation errors small compared to $d_{\theta}=$ $\pi / 2$ are detectable with a code-dependent uncertainty.

$\left\{m d_{\theta}\right\}$ for $m=0,1, \ldots, 2 N-1$ as the phase grid and the set of Fock states $\{|k N\rangle\}$ for $k=0,1, \ldots$ as the Fock grid. These are in analogy to the position- and momentum-space grids (or more general quadrature grids) on which GKP codes are defined. They broadly characterize the types of error to which the code is naturally resilient. A number-shift error smaller than $d_{n}$ [e.g., $\sim \hat{a}^{k}$ or $\sim\left(\hat{a}^{\dagger}\right)^{k}$ with $\left.k<N\right]$ is detectable. For rotation errors, the boundary between detectable and undetectable errors is not as sharp, in general, but a good code should be able to detect a rotation which is small compared to $d_{\theta}$ (e.g., $e^{i \theta \hat{n}}$ with $\theta<d_{\theta}$ ). This basic idea is illustrated in Fig. 2. Note that the spacing in Fock space and in terms of rotations, as quantified by Eq. (13), is not by itself enough to guarantee a code's ability to correct errors. This ability depends on both the noise channel in question and the Fock grid coefficients $f_{k N}$ (or, equivalently, the primitive $|\Theta\rangle$ ) parametrizing the code [Eq. (4)]. In fact, the bosonic codes we investigate in this paper are, in general, only approximate error-correction codes for the noise channels we are interested in, and one can typically talk about only "approximately correctable errors" $[47,48]$. We return to a more detailed discussion of "small" vs "large" errors in Sec. VI.

\section{Distinguishing the code words}

The structure of rotation codes in Fock space [Eq. (4)] and in terms of rotations [Eq. (3)] suggests a natural way to distinguish code words in the computational and dual bases using number and phase measurements, respectively. Phase 
measurements play a crucial role in the quantum computing scheme introduced in Sec. IV.

\section{Number measurement}

The computational-basis code words $\left|0_{N}\right\rangle$ and $\left|1_{N}\right\rangle$ can be distinguished (destructively) by measuring the excitation number as seen clearly in Eq. (4). For an order- $N$ code, a number measurement returns an outcome $k N$ for integer $k$, where even $k$ corresponds to $\left|0_{N}\right\rangle$ and odd $k$ to $\left|1_{N}\right\rangle$. In the presence of errors, such a measurement can still correctly identify the damaged code words. For example, if we assume that upward and downward shifts in number (e.g., loss and gain) are equally likely, a simple decoder to identify the code word from a number measurement outcome $n$ is to round to the nearest $k N$ and declare the outcome " 0 " if $k$ is even and " 1 " if $k$ is odd. In practice, the noise model motivates the decoding scheme. Under pure loss, for example, the decoder should always round $u p$ to the nearest $k N$.

\section{Phase measurement}

An approach to distinguishing $\left| \pm_{N, \Theta}\right\rangle$ with a natural robustness to rotation errors relies on phase estimation. In the absence of errors, the task can be formulated as follows: Given the code word $\left|+_{N, \Theta}\right\rangle$ for a rotation code, we wish to determine $\theta$ in $e^{i \theta \hat{n}}\left|+_{N, \Theta}\right\rangle$. If $\theta \bmod 2 \pi / N=0$, the state is $\left|+_{N, \Theta}\right\rangle$, while if $\theta \bmod 2 \pi / N=\pi / N$, the state is $\left|-_{N, \Theta}\right\rangle$. In the presence of rotation errors, the phase-estimation problem is generalized to estimating a continuous variable $\theta \in[0,2 \pi)$. A decoding procedure rounds $\theta$ to the nearest $m \pi / N$. Then, if $m$ is even, declare the outcome " + ," and if $m$ is odd, declare the outcome "-." This procedure is exactly analogous to the excitation number decoding scheme described above. Again, the decoding scheme should, in general, be adapted to the system's noise model.

Holevo [32] and Helstrom [49] introduced an optimal measurement to distinguish states in the one-parameter family $e^{i \theta \hat{n}}\left|\psi_{0}\right\rangle$ for the situation where no prior information about $\theta$ is assumed (and for a specific class of "deviation functions" quantifying the deviation of the estimated value from the true value). The positive operator-valued measure (POVM) elements of this measurement depend on a fiducial state $\left|\psi_{0}\right\rangle=\sum_{n} c_{n}|n\rangle$ that defines the phaseestimation problem. They can be written in the Fock basis (Theorem 4.4.1 in Ref. [32]):

$$
\hat{M}^{\mathrm{can}}(\theta):=\frac{1}{2 \pi} \sum_{m, n=0}^{\infty} \gamma_{m} \gamma_{n}^{*} e^{i(m-n) \theta}|m\rangle\langle n|,
$$

with completeness relation $\int_{0}^{2 \pi} d \theta \hat{M}^{\text {can }}(\theta)=\hat{I}$, and $\gamma_{n}=$ $c_{n} /\left|c_{n}\right|$ for $c_{n} \neq 0$ and otherwise an arbitrary complex number with $\left|\gamma_{n}\right|=1$. In the present context, $\left|\psi_{0}\right\rangle=$ $\left|+_{N, \Theta}\right\rangle=(1 / \sqrt{2}) \sum_{k} f_{k N}|k N\rangle$. We refer to the set of POVMs in Eq. (14) as the canonical phase measurement.
For an order- $N$ rotation code, the relation $\hat{R}_{N}\left|+_{N, \Theta}\right\rangle=$ $\left|+_{N, \Theta}\right\rangle$ implies that phase measurements acquire only information about the phase $\theta$ modulo $2 \pi / N$. To quantify the uncertainty in a phase-estimation measurement where we care only about the modular phase, we slightly modify a phase-uncertainty measure introduced by Holevo [32]. We adopt a measure of modular phase uncertainty by considering the $2 \pi / N$ periodic random variable $e^{i N \theta}$ under the probability distribution $\mu(\theta)=\operatorname{tr}\left[\hat{M}^{\text {can }}(\theta)\left|+_{N, \Theta}\right\rangle\left\langle+_{N, \Theta}\right|\right]$ for $\theta$. We can then define an uncertainty measure

$$
\Delta_{N}(\theta):=\frac{\left\langle\Delta e^{i N \theta}\right\rangle}{\left|\left\langle e^{i N \theta}\right\rangle\right|^{2}}=\frac{1}{\left|\left\langle e^{i N \theta}\right\rangle\right|^{2}}-1,
$$

where $\left\langle e^{i N \theta}\right\rangle:=\int_{0}^{2 \pi} e^{i N \theta} \mu(\theta) d \theta$ is the mean modular phase and $\left\langle\Delta e^{i N \theta}\right\rangle:=\int_{0}^{2 \pi}\left|e^{i N \theta}-\left\langle e^{i N \theta}\right\rangle\right|^{2} \mu(\theta) d \theta$.

Even in the absence of external noise, the underlying primitive endows a code with some level of modular phase uncertainty. This uncertainty can be found from Eq. (15) using the following form for the mean modular phase:

$$
\left\langle e^{i N \theta}\right\rangle=\int_{0}^{2 \pi} e^{i N \theta} \mu(\theta) d \theta=\frac{1}{2} \sum_{k=0}^{\infty}\left|f_{k N} f_{(k+1) N}\right|,
$$

where $f_{k N}$ are the Fock-grid coefficients of the code [Eq. (4)], as before. Henceforth, we use $\Delta_{N}(\theta)$ to quantify the embedded phase uncertainty in the code words. This embedded uncertainty is in analogy to the embedded position or momentum errors in finitely squeezed GKP code words $[3,50]$. Note that, in general, the embedded phase uncertainty depends on the order of rotation symmetry. In Sec. III, we show that certain families of rotation codes have meaningful limits where $\Delta_{N}(\theta) \rightarrow 0$, which is akin to the limit of infinite squeezing for ideal GKP codes.

Note that, because the canonical measurement Eq. (14) assumes no prior knowledge about $\theta$, it might be suboptimal if such knowledge exists. If rotation errors are small, we expect that $\theta$ is close to one of the values $m \pi / N$, and it might be possible to exploit this knowledge in a phase-estimation measurement. Moreover, since the measurement is defined with respect to the ideal, undamaged code word $\left|+_{N, \Theta}\right\rangle$, it might perform less than ideally in the presence of general noise that includes errors beyond pure rotation errors. For the numerical simulations of errorcorrection circuits in Sec. VII B, we compare the canonical phase measurement to "pretty good measurements," as introduced in Refs. [51-53] (see also Appendix D), which explicitly take information about the noise model into account.

When all $\gamma_{n}$ are real in Eq. (14), the measurement can be approximated using adaptive homodyne measurements [54] and has been the subject of much theoretical and experimental study [55-61]. One can similarly define phase-uncertainty measures analogous to Eq. (15) for other 
(a)

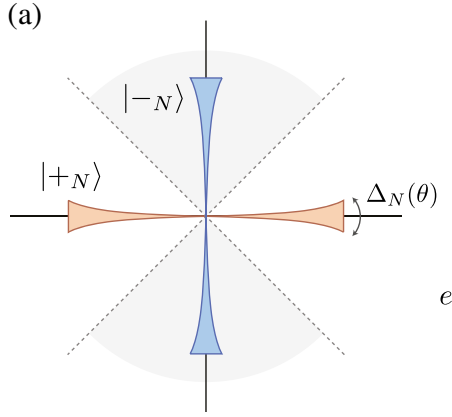

(b)

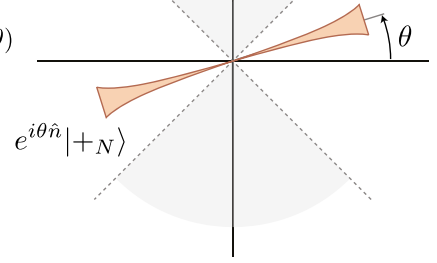

FIG. 3. Distinguishing the $\left| \pm_{N}\right\rangle$ code words. By design, $\left|-_{N}\right\rangle=e^{i(\pi / N) \hat{n}}\left|+_{N}\right\rangle$, such that distinguishing the code words can be viewed as a phase-estimation problem. The phase precision $\Delta_{N}(\theta)$ depends on the measurement scheme, with a canonical scheme given by the POVM in Eq. (14). (a) Measuring $\theta$ to distinguish the code words for an $N=2$ code $\left(d_{\theta}=\pi / 2\right)$. If the measurement result falls in a white wedge, we declare "+," and in a gray wedge, we declare "-." (b) The same procedure for a code word that has undergone a rotation error $e^{i \theta \hat{n}}\left|+_{N}\right\rangle$. For large rotations $\theta \sim d_{\theta} / 2$, the logical state is misidentified. If the noise model is biased in such a way that clockwise rotations are more likely than counterclockwise (or vice versa), the decoder should be biased accordingly by rotating the white and gray wedges.

phase-estimation schemes. As an example, a heterodyne measurement also measures the phase but at the cost of larger uncertainty. An ideal heterodyne measurement is described by measurement operators $|\alpha\rangle\langle\alpha|$, where $|\alpha\rangle$ is a coherent state, with completeness relation $\int\left(d^{2} \alpha / \pi\right)|\alpha\rangle\langle\alpha|=\hat{I}$. The estimated phase for an outcome $\alpha$ is simply $\theta=\arg (\alpha)$. We can define a phase uncertainty $\Delta_{N}^{\text {het }}(\theta)$ for a heterodyne measurement analogously to the canonical phase uncertainty defined above. A heterodyne measurement gives a larger uncertainty in the estimated phase than the canonical phase measurement; however, a heterodyne measurement is nevertheless a likely practical candidate for distinguishing the logical code words of the number-phase codes introduced in the next section.

In general, the phase uncertainty in a measurement is a function of both the code words and the choice of measurement scheme. As long as the phase fluctuations in the measurement results are small compared to the rotational distance $d_{\theta}=\pi / N$, the two code words $\left| \pm_{N, \Theta}\right\rangle$ can be distinguished faithfully. This idea is illustrated in Fig. 3.

\section{NUMBER-PHASE CODES}

The embedded phase uncertainty in Eq. (15), $\Delta_{N}(\theta)$, is minimized when the distribution of Fock-grid coefficients is completely flat, i.e., $\left|f_{k N}\right|=\left|f_{(k+1) N}\right|$ for all $k$, such that $\left|\left\langle e^{i N \phi}\right\rangle\right|=1$ and $\Delta_{N}(\theta)=0$; cf. Eq. (15)(16). However, such a state is not normalizable, rendering this limit unphysical. Formally, we can consider families of physical codes that in some appropriate limit satisfy $\Delta_{N}(\theta) \rightarrow 0$. As we show in the following, this condition is satisfied for a class of rotation codes very much analogous to GKP codes, with number and phase playing roles akin to position and momentum. Perhaps somewhat surprisingly, cat and binomial codes both belong to this class [62].

\section{A. Ideal number-phase codes}

In analogy with ideal GKP codes defined in terms of superpositions of position (or momentum) eigenstates, we define a family of order- $N$ rotation codes as

$$
\begin{gathered}
\left|0_{N, \text { phase }}\right\rangle:=\sum_{m=0}^{2 N-1}\left|\phi=\frac{m \pi}{N}\right\rangle, \\
\left|1_{N, \text { phase }}\right\rangle:=\sum_{m=0}^{2 N-1}(-1)^{m}\left|\phi=\frac{m \pi}{N}\right\rangle,
\end{gathered}
$$

where $|\phi\rangle:=(1 / \sqrt{2 \pi}) \sum_{n=0}^{\infty} e^{i n \phi}|n\rangle$ are known as phase states and are not normalizable $[67,68]$. In the Fock basis, the code words are simply $\left|0_{N}\right\rangle \propto \sum_{k}|2 k N\rangle$ and $\left|1_{N}\right\rangle \propto$ $\sum_{k}|(2 k+1) N\rangle$. Note that we can write the POVM for the canonical phase measurement in Eq. (14) (for real coefficients $\gamma_{n}=1$ ) as $\hat{M}^{\text {can }}(\phi)=|\phi\rangle\langle\phi|$. Equation (17) follows from Eq. (3) by replacing $|\Theta\rangle \propto|\phi=0\rangle$.

Any state in the code space spanned by Eq. (17) is, of course, a +1 eigenstate of $\hat{R}_{N}$. The code words are also +1 eigenstates of a number-translation operator

$$
\hat{\Sigma}_{N}:=\sum_{n=0}^{\infty}|n\rangle\langle n+2 N| .
$$

We have that $\hat{R}_{N} \hat{\Sigma}_{N}=\hat{\Sigma}_{N} \hat{R}_{N}$, and the operator

$$
\hat{X}_{N}:=\hat{\Sigma}_{N / 2}=\sum_{n=0}^{\infty}|n\rangle\langle n+N|
$$

satisfies $\hat{X}_{N} \hat{Z}_{N}=-\hat{Z}_{N} \hat{X}_{N}$, while both $\hat{Z}_{N}$ and $\hat{X}_{N}$ commute with $\hat{R}_{N}$ and $\hat{\Sigma}_{N}$. Finally, for the code Eq. (17), we have that $\hat{X}_{N}\left| \pm_{N}\right\rangle= \pm\left| \pm_{N}\right\rangle$, and this operator thus acts as logical $\bar{X}$.

\section{B. Approximate number-phase codes}

Ideal number-phase codes as defined in Eq. (17) are unphysical, as they require an infinite excitation number and are not normalizable. Nevertheless, certain families of rotation codes approach ideal number-phase codes, in a sense to be defined below, in limits of a large excitation number.

We first recognize that, for any rotation code with positive, real Fock-grid coefficients $\left\{f_{k N}\right\}$ [Eq. (4)], we have $\left\langle \pm_{N, \Theta}\left|\hat{X}_{N}\right| \pm_{N, \theta}\right\rangle= \pm\left\langle e^{i N \theta}\right\rangle$. As a consequence,

$$
\left\langle \pm_{N, \Theta}\left|\hat{X}_{N}\right| \pm_{N, \theta}\right\rangle \rightarrow \pm 1
$$


TABLE I. Three codes — cat, binomial, and Pegg-Barnett—whose phase uncertainty vanishes in an appropriate limit (see Appendix B for definitions and details of each code). The second and third columns give a primitive and the Fock-grid coefficients [Eq. (4)], respectively (for cat codes, $\mathcal{N}_{i}^{*}$ is the normalization factor $\mathcal{N}_{0}^{*}$ for even $k$ and $\mathcal{N}_{1}^{*}$ for odd $k$ ). The fourth column shows the limit where each code becomes the $0 \mathrm{~N}$ encoding (which is the trivial encoding [Eq. (12)] for $N=1$ ), and the fifth gives the limit where $\Delta_{N}(\theta) \rightarrow 0$. The sixth column gives an asymptotic form of the Fock-grid coefficients, and the last column gives an asymptotic form for the mean modular phase. For each code, the mean modular phase uncertainty approaches zero: $\Delta_{N}(\theta) \rightarrow 0$. The function $\vartheta_{3}(r, \tau)$ is the Jacobi elliptic theta function of the third kind.

\begin{tabular}{|c|c|c|c|c|c|c|}
\hline Code & $|\Theta\rangle$ & $f_{k N}$ & $\begin{array}{l}\text { ON } \\
\text { code }\end{array}$ & Limit & Limiting $f_{k N}$ & $\begin{array}{c}\text { Limiting mean } \\
\text { modular phase }\left\langle e^{i \theta N}\right\rangle\end{array}$ \\
\hline cat & $|\alpha\rangle$ & $\sqrt{\frac{2}{\mathcal{N}_{i}^{*}}} \frac{e^{-|\alpha|^{2} / 2} \alpha^{k N}}{\sqrt{(k N) !}}$ & $\alpha \rightarrow 0$ & $\alpha \rightarrow \infty$ & $\left(\frac{2 N^{2}}{\pi \alpha^{2}}\right)^{1 / 4} \exp \left[\frac{-\left(k N-\alpha^{2}\right)^{2}}{4 \alpha^{2}}\right]$ & $e^{-\frac{N^{2}}{8 \alpha^{2}}} \vartheta_{3}\left[\frac{N \pi}{2}\left(1-\frac{2 \alpha^{2}}{N}\right), e^{-2 \pi^{2} \frac{\alpha^{2}}{N^{2}}}\right]$ \\
\hline binomial & $\left|\Theta_{\mathrm{bin}}^{N, K}\right\rangle$ & $\sqrt{\frac{1}{2^{K-1}}\left(\begin{array}{l}K \\
k\end{array}\right)}$ & $K=1$ & $K \rightarrow \infty$ & $\left(\frac{8}{\pi K}\right)^{1 / 4} \exp \left[\frac{-(k-K / 2)^{2}}{K}\right]$ & $e^{-\frac{1}{2 K}} \vartheta_{3}\left[\frac{\pi}{2}(1-K), e^{-\frac{K \pi^{2}}{2}}\right]$ \\
\hline Pegg-Barnett & $|\phi=0, s\rangle$ & $\sqrt{2 /\lceil s / N\rceil}$ & $s=N+1$ & $s \rightarrow \infty$ & $\sqrt{2 /\lceil s / N\rceil}$ & $1-1 /\lceil s / N\rceil$ \\
\hline
\end{tabular}

if and only if $\Delta_{N}(\theta) \rightarrow 0$, where $\Delta_{N}(\theta)$ and $\left\langle e^{i N \theta}\right\rangle$ are defined in Eqs. (15) and (16), respectively. It follows that the condition of vanishing phase uncertainty defines a family of codes that, in this limit, are stabilized by $\hat{\Sigma}_{N}$ [Eq. (18)] and for which $\hat{X}_{N}$ [Eq. (19)] acts as logical $\bar{X}$.

An interesting property of number-phase codes arises from the fact that, in the limit of Eq. (20), the two code-word normalization constants are equal $\left(\mathcal{N}_{0}=\mathcal{N}_{1}\right)$ in Eq. (3). It follows that the dual-basis code words also become equalweight superpositions of the primitive [see Eq. (A4)]:

$$
\begin{gathered}
\left|+_{N, \Theta}\right\rangle \rightarrow \frac{1}{\sqrt{N}} \sum_{m=0}^{N-1} e^{i(2 m \pi / N) \hat{n}}|\Theta\rangle, \\
\left|-_{N, \Theta}\right\rangle \rightarrow \frac{1}{\sqrt{N}} \sum_{m=0}^{N-1} e^{i[(2 m+1) \pi / N] \hat{n}}|\Theta\rangle .
\end{gathered}
$$

A stronger number-phase duality is apparent for numberphase codes than for generic rotation codes: The dual-basis code words $\left| \pm_{N}\right\rangle$ are separated from each other by exactly $d_{\theta}=\pi / N$ in terms of phase-space rotations but have support on the same Fock states; cf. Eq. (9). Conversely, the computational-basis code words $\left|0_{N} / 1_{N}\right\rangle$ are separated from each other by $d_{n}=N$ in Fock space but supported on the same set of phases $\{m \pi / N\}$.

There are many examples of rotation codes that limit to ideal number-phase codes in the sense of Eq. (20). A straightforward example is codes constructed using normalized Pegg-Barnett phase states, $|\phi, s\rangle:=$ $(1 / \sqrt{s}) \sum_{n=0}^{s-1} e^{i n \phi}|n\rangle[64,66,69]$, as the primitive $|\Theta\rangle$. As the parameter that sets their truncation in Fock space becomes large $(s \rightarrow \infty)$, Pegg-Barnett codes approach ideal number-phase codes (up to normalization). With the constraint $s=p \times 2 N$ for integer $p \geq 1$, the Pegg-Barnett codes are identical to the shift-resistant qudit codes introduced in Ref. [3], embedded in the infinite Hilbert space of a bosonic mode [70]. Further details about PeggBarnett codes can be found in Appendix B 3.
Cat codes (Appendix B 1) and binomial codes (Appendix B 2) also satisfy Eq. (20) in the limit of large $\alpha$ and large truncation $K$, respectively. Table I summarizes the three examples of number-phase codes we
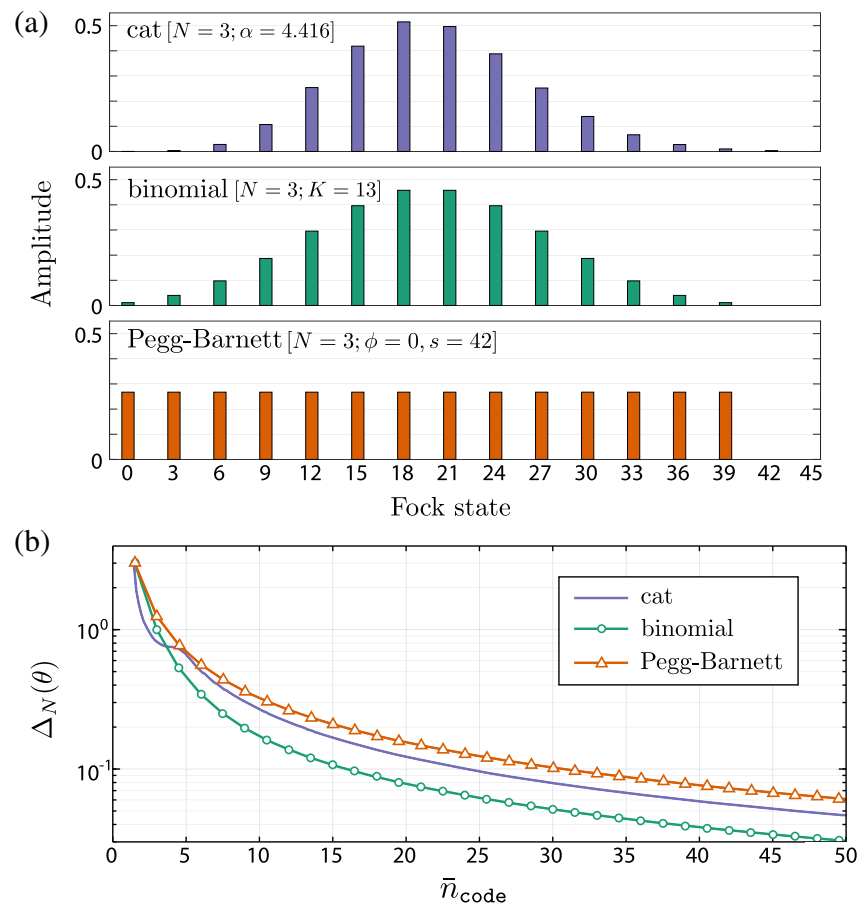

FIG. 4. (a) Magnitude of the Fock-state amplitudes for a $\left|+{ }_{N}\right\rangle$ state for $N=3$ cat, binomial, and Pegg-Barnett codes with parameters that give $\bar{n}_{\text {code }}=19.5$ for each. The amplitudes are nonzero only on Fock states $|3 k\rangle$ for $k=0,1,2, \ldots$ (b) The embedded phase uncertainty [Eq. (15)] for each family of $N=3$ codes as $\bar{n}_{\text {code }}$ increases. For each code, a proper asymptotic limit, given in Table I, yields no embedded phase uncertainty, $\Delta_{N}(\theta) \rightarrow 0$. In the opposite regime, $\bar{n}_{\text {code }} \rightarrow \frac{3}{2}$, all three codes limit to the 03 encoding. Note that $N=3$ codes with $\bar{n}_{\text {code }}<\frac{3}{2}$ do not exist and that binomial and Pegg-Barnett codes are defined only at discrete excitation numbers; connecting lines are guides for the eye. 
discuss here. For these codes, the embedded phase uncertainty approaches zero when the relevant code parameter is large enough, as shown in Fig. 4 for some example $N=3$ codes.

Colloquially, we refer to any family of rotation codes that satisfies $\Delta_{N}(\theta) \rightarrow 0$ in an appropriate limit as a numberphase code. In practice, the specific form of the Fock-grid coefficients $\left\{f_{k N}\right\}$ can make a significant difference in the embedded phase uncertainty for small to moderate excitation numbers. Phase measurements play a critical role in the quantum computing scheme of Sec. IV and the error-correction procedures of Sec. VII. As a consequence, although different number-phase codes behave identically for large excitation numbers, they can have different performance at experimentally relevant excitation numbers [44]. This difference is apparent in Sec. VII B, where we compare the error-correction performance of cat and binomial codes.

\section{SYMMETRY AS A RESOURCE: QUANTUM COMPUTING WITH NUMBER-PHASE CODES}

We introduce a quantum computing scheme based on three basic ingredients: preparation of code words in the state $\left|+_{N}\right\rangle$, a small number of unitary gates, and (destructive) measurement in the logical $\bar{X}$ basis. Our focus is on operations that are, in a certain sense, fault tolerant. For unitary gates, fault tolerance means that the gates should not amplify errors too badly, a notion that is made precise later in Sec. VI, while for the measurement it means that the code words $\left| \pm_{N}\right\rangle$ should be distinguishable in the presence of small errors. As already discussed in Sec. II C 2, the latter can be achieved with phase measurements, given that the codes have a small inherent phase uncertainty. Vanishing phase uncertainty is equivalent to (approximate) translation symmetry in Fock space, as we showed in Sec. III, such that the existence of a robust $\bar{X}$-basis measurement can be said to be a consequence of this number-translation symmetry. On the other hand, the unitary gates we introduce rely only on rotation symmetry. We show that, given a supply of encoded basis states $\left|+_{N}\right\rangle$, these two symmetries imply the ability to enact any gate in the logical Clifford group, using physically reasonable operations that are fault tolerant in the above sense. The situation can be compared to GKP codes, where (approximate) discrete quadrature translation symmetry leads to any logical Clifford being (approximately) implementable by a Gaussian operation [3].

While state preparation is in general code dependent, flexible techniques have already been developed in various physical platforms, such as circuit QED [29,30] and ion traps [71], that can be applied to prepare code words for a wide range of codes. It is beyond the scope of the present work to discuss state preparation in detail, but we briefly comment on a few approaches in Sec. IV B. All of the known approaches are limited by noise in the preparation procedure, and one cannot rule out code words with large errors. The issue of faulty state preparation is postponed until Sec. VIII, and for the time being we simply assume that $\left|+{ }_{N}\right\rangle$ states can be prepared.

Because of the existence of a robust $\bar{X}$-basis measurement, our main focus is on approximate number-phase codes from here on. That said, the unitary gates introduced below apply to any rotation code, such that the scheme can be extended to any such code where a practical $\bar{X}$-basis measurement can be found.

\section{A. Universal operations for number-phase codes}

The quantum computing scheme introduced in the following is based on the universal set

$$
\left\{\bar{S}, \bar{C}_{Z}\right\} \cup\left\{\mathcal{P}_{\left|+_{N}\right\rangle}, \mathcal{P}_{\left|T_{N}\right\rangle}, \mathcal{M}_{X}\right\},
$$

where $\bar{S}$ is an encoded version of the phase gate $\hat{S}=\operatorname{diag}(1, i), \bar{C}_{Z}$ is an encoded $\hat{C}_{Z}=\operatorname{diag}(1,1,1,-1)$, $\mathcal{P}_{\left|\psi_{N}\right\rangle}$ stands for preparation of an encoded state $\left|\psi_{N}\right\rangle$, and $\mathcal{M}_{X}$ stands for measurement in the logical $\left| \pm_{N}\right\rangle$ basis. The state $\left|T_{N}\right\rangle \propto\left|0_{N}\right\rangle+e^{i \pi / 4}\left|1_{N}\right\rangle$ is used for gate teleportation of the non-Clifford $\hat{T}=\operatorname{diag}\left(1, e^{i \pi / 4}\right)$ gate.

A remarkable feature of the following scheme is that the required unitary gates are agnostic to the specific details of the rotation code in question. The encoded $\left\{\bar{S}, \bar{C}_{Z}\right\}$ gates depend only on the degree of rotation symmetry and, consequently, apply equally to all rotation codes. This feature allows entangling operations between different rotation codes, e.g., cat and binomial, and teleportation from one code to another.

\section{Unitary gates}

We have already shown that logical $\bar{Z}$ for an order- $N$ rotation code can be implemented through a simple rotation $\hat{Z}_{N}=e^{i \pi \hat{n} / N}$. The next gate in our set is the following twomode gate that implements a controlled rotation between an order- $N$ and an order- $M$ rotation code [2,72]:

$$
\mathrm{CROT}_{N M}:=e^{i(\pi / N M) \hat{n} \otimes \hat{n}} .
$$

To understand its action on the code space, we recognize that $\mathrm{CROT}_{N M}|k N\rangle \otimes|l M\rangle=e^{i \pi k l}|k N\rangle \otimes|l M\rangle$ for Fock states $|k N\rangle$ and $|l M\rangle$. Since $k l$ is even unless both $k$ and $l$ are odd, it follows from Eq. (4) that the action on an encoded state $\left|i_{N}\right\rangle \otimes\left|j_{M}\right\rangle$ is

$$
\mathrm{CROT}_{N M}\left|i_{N}\right\rangle \otimes\left|j_{M}\right\rangle=(-1)^{i j}\left|i_{N}\right\rangle \otimes\left|j_{M}\right\rangle
$$

for $i, j=0,1$. Thus, the CROT gate acts as a $\bar{C}_{Z}$ gate on the code space of any two rotation codes.

A logical $\hat{S}=\operatorname{diag}(1, i)$ can similarly be enacted by a quartic (in $\hat{a}, \hat{a}^{\dagger}$ ) single-mode Hamiltonian:

$$
\bar{S}=\hat{S}_{N}:=e^{i\left(\pi / 2 N^{2}\right) \hat{n}^{2}} .
$$


The action of $\hat{S}_{N}$ on the code space can be seen by acting on the Fock states in Eq. (4): $\hat{S}_{N}|2 k N\rangle=e^{(i \pi / 2) 4 k^{2}}|2 k N\rangle=$ $|2 k N\rangle$, since $2 k^{2}$ is even. On the other hand, $\hat{S}_{N} \mid(2 k+$ 1) $N\rangle=e^{(i \pi / 2)\left(4 k^{2}+4 k+1\right)}|(2 k+1) N\rangle=i|(2 k+1) N\rangle$.

In principle, Hamiltonians of even higher order in $\hat{n}$ can be used to generate diagonal non-Clifford gates. In particular, a logical $\bar{T}$ gate could be enacted by

$$
\hat{T}_{N}:=e^{i\left(\pi / 4 N^{4}\right) \hat{n}^{4}},
$$

as is easily checked by acting on the code words $\left|0_{N}\right\rangle$ and $\left|1_{N}\right\rangle$ in Eq. (4). However, in addition to the experimental challenge of engineering an octic Hamiltonian, the $\hat{T}_{N}$ gate can amplify errors in damaging ways as we show in Sec. VI. It is therefore unlikely to be of practical use, and we propose a scheme based on gate teleportation instead.

\section{Teleported gates}

The CROT gate and logical $\mathcal{M}_{X}$ measurements, together with appropriately prepared ancillae, allow us to complete a universal set of operation with a gate-teleported logical Hadamard $\bar{H}$ and $\bar{T}$ gate.

An ancilla prepared in $\left|+_{M}\right\rangle$ allows execution of the Hadamard gate, using the following teleportation circuit $[36,73,74]$ :

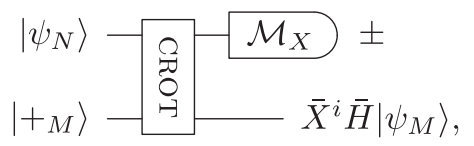

where $\left|\psi_{N}\right\rangle=a\left|0_{N}\right\rangle+b\left|1_{N}\right\rangle$ and $\left|\psi_{M}\right\rangle=a\left|0_{M}\right\rangle+b\left|1_{M}\right\rangle$ represent the same encoded qubit state (but not necessarily in the same code). The measurement is in the $\left| \pm_{N}\right\rangle$ basis, and we use $i=0$ for the outcome " + " and $i=1$ for the outcome "-." The notation $\bar{X}^{i} \bar{H}$ thus means that, depending on the outcome, an $\bar{H}(+)$ or $\bar{X} \bar{H}(-)$ is applied to the data. Since our scheme for quantum computation is based only on Clifford gates and magic-state injection, rather than correcting the conditional Pauli $\bar{X}$, the simplest solution is to keep track of it in a Pauli frame $[33,75]$. Alternatively, the process can be repeated until the desired outcome is achieved [73].

The set $\left\{\bar{C}_{Z}, \bar{H}, \bar{S}\right\}$ generates the Clifford group. To achieve universality, we teleport the gate $\hat{T}=\operatorname{diag}\left(1, e^{i \pi / 4}\right)$ by consuming an ancilla prepared in $\left|T_{M}\right\rangle=\left(\left|0_{M}\right\rangle+\right.$ $\left.e^{i \pi / 4}\left|1_{M}\right\rangle\right) / \sqrt{2}[76]:$

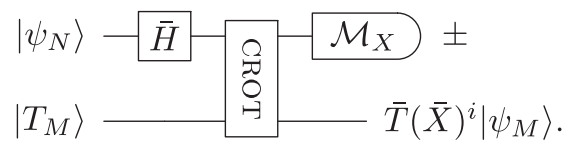

In order to teleport the $\bar{T}$ gate, we need an encoded $\left|T_{M}\right\rangle$ state. Arbitrary encoded states can be injected assuming we have universal control over two-level ancillae in the trivial encoding $\left|0_{\text {triv }}\right\rangle=|0\rangle,\left|1_{\text {triv }}\right\rangle=|1\rangle$ : The circuit in Eq. (27) can be used to teleport an arbitrary ancilla state into a rotation code, where the top rail represents the two-level ancilla with $N=1$. The procedure is not fault tolerant, because an error on the ancilla propagates into the rotation code. Preparation of $|T\rangle$ states can be made fault tolerant using magic-state distillation, assuming high-quality Clifford operations are available [78,79]. As we discuss in Sec. VIII, this result can be achieved by concatenation with a second code and performing state distillation at the level of the top code.

If needed, we can also execute an $\hat{S}=\operatorname{diag}(1, i)$ gate in a similar fashion by consuming an ancilla prepared in $\left|+i_{M}\right\rangle=\left(\left|0_{M}\right\rangle+i\left|1_{M}\right\rangle\right) / \sqrt{2}$ :

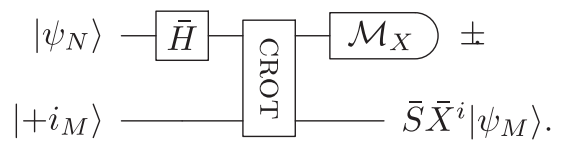

Again, the outcome-dependent factor $\bar{X}^{i}$ can be kept track of using a Pauli frame. The circuit in Eq. (29) can be an alternative to using the unitary gate in Eq. (42) directly, which might be more practical experimentally than controlling a self-Kerr Hamiltonian $\sim \hat{n}^{2}$.

\section{B. State preparation}

State preparation is a crucial ingredient for quantum computing with bosonic codes and can also be the most difficult part. Here, the logical $\left|+_{N}\right\rangle$ state plays a key role in both the gate teleportation and state injection needed for teleported $\bar{T}$ gates. A large literature exists on state preparation of GKP code words [50,80-83], while relatively less theoretical work has been done for rotation codes, and the optimal way to prepare code words, in general, depends on the code in question. This topic is therefore broad, and we do not address it in detail in this work.

One experimental approach to preparing logical basis states involves a strong dispersive interaction between the mode and an ancilla qubit (in circuit QED, these could be a cavity mode and a transmon qubit). Combined with displacement operations, such an interaction is, in principle, universal $[84,85]$, in the sense that any state of the mode can be prepared in the absence of errors. Optimal control can be used to generate a sequence of control operations that maximizes the fidelity of a target state in the presence of errors, which has been performed in the circuit QED setting [29,30]. An alternate approach could be to prepare a primitive $|\Theta\rangle$ and then measure excitation number mod $N$, using the measurement introduced below in Sec. V. For either of these approaches, the fidelity of the state preparation is limited by noise in the preparation procedure, and one cannot rule out corrupted code words 
with large errors. We postpone a discussion of how to deal with faulty state preparation until Sec. VIII.

\section{Breeding rotation symmetry}

We here present a method to "breed" logical states for an order- $N$ rotation code from code states with lower order rotation symmetry. In analogy to a scheme for producing GKP states from Ref. [80], each stage of the breeding consists of coupling the bosonic mode to an ancilla mode followed by measurement of the ancilla. When successful, a $\left|0_{2 N, \Theta}\right\rangle$ state is produced from a $\left|0_{N, \Theta}\right\rangle$ state, and the whole process can be repeated as required.

We begin with a code word $\left|0_{N . \Theta}\right\rangle$ and an ancilla qubit prepared in a state $\left|{ }_{M}\right\rangle$ coupled via CROT interaction

$$
\mathrm{CROT}_{2 N, M}=e^{i(\pi / 2 N M) \hat{n} \otimes \hat{n}} .
$$

Critically, we note that the ancilla could be a two-level system in the state $\left|+_{\text {triv }}\right\rangle=(|0\rangle+|1\rangle) / \sqrt{2}(M=1)$ or encoded in a rotation code of any order $M$. The ancilla is then measured in the $\left| \pm_{M}\right\rangle$ basis. When the + outcome is obtained, the state becomes superposed with a rotated version to create a code word of higher rotation symmetry:

$$
\frac{1}{2}\left(\hat{I}+\hat{Z}_{2 N}\right)\left|0_{N, \Theta}\right\rangle=\sqrt{\mathcal{P}_{+}}\left|0_{2 N, \Theta}\right\rangle,
$$

with outcome probability $\mathcal{P}_{+}=\frac{1}{2}\left(1+\left\langle 0_{N, \Theta}\left|\hat{Z}_{2 N}\right| 0_{N, \Theta}\right\rangle\right)$. Beginning from a primitive $|\Theta\rangle, n$ successful rounds of breeding produce a logical state with $2^{n}$-fold rotation symmetry, $\left|0_{2^{n}, \Theta}\right\rangle$. Just as in Ref. [80], the success probability falls off exponentially in $n$, but this probability could be improved by making use of higher-dimensional ancillae such as in Ref. [50]. The relation Eq. (31) can likewise be used on a low-order $\left|+_{N, \Theta}\right\rangle$ (or a dual primitive-see Appendix A) to breed a higher-order $\left|+_{2 N, \Theta}\right\rangle$ state.

It is also worth noting here that for number-phase codes discussed in Sec. III, which are our main focus, we have the approximate relation (see also Appendix A)

$$
\left|0_{N, \Theta}\right\rangle \simeq\left|+_{2 N, \Theta}\right\rangle
$$

Thus, preparation of $\left|0_{N, \Theta}\right\rangle$ states is equivalent to preparation of approximate $\left|+{ }_{2 N, \Theta}\right\rangle$ states for these codes.

\section{MODULAR NUMBER MEASUREMENT}

The CROT gate is a fundamental building block for the universal gate set, state breeding, and the error-correction scheme we introduce in Sec. VII. Here, we show that the CROT gate can also be used to perform a nondestructive modular measurement of excitation number $\hat{n}$. In addition to being a novel measurement for a bosonic mode, the modular number measurement can be used both to detect number-shift errors on an encoded state and to conditionally prepare $N$-fold rotation-symmetric code words from a primitive $|\Theta\rangle$. The measurement we present here is a natural generalization of previous schemes where a two-level ancilla (transmon qubit) is used to detect the number mod 2 for cat and binomial codes [29,30].

A nondestructive measurement of $\hat{n} \bmod N$ can be performed with the following circuit:

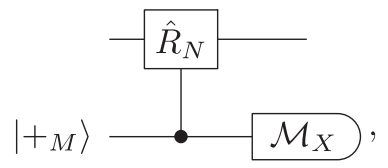

where the controlled- $\hat{R}_{N}$ gate is defined as

$$
\hat{C}_{R_{N}}:=\mathrm{CROT}_{N M / 2}=e^{i(2 \pi / N M) \hat{n} \otimes \hat{n}},
$$

and the measurement is to be understood as a phase measurement as described in Sec. II C 2. To see how this circuit works, consider the action of the $\hat{C}_{R_{N}}$ gate on a Fock state $|n\rangle$ for the data rail and $\left|+_{M}\right\rangle$ for the ancilla:

$$
\hat{C}_{R_{N}}|n\rangle \otimes\left|+_{M}\right\rangle=|n\rangle \otimes e^{i(\ell / N)(2 \pi / M) \hat{n}}\left|+{ }_{M}\right\rangle,
$$

where $\ell:=n \bmod N$ and we use that $e^{i(2 \pi p / M) \hat{n}}\left|{ }_{M}\right\rangle=$ $\left(\hat{R}_{M}\right)^{p}\left|+_{M}\right\rangle=\left|+_{M}\right\rangle$ for any integer $p$. The net result is a rotation of the ancilla state $\left|+_{M}\right\rangle$ by an angle $\theta_{\mathrm{anc}}=2 \pi \ell /(N M)$, which takes the ancilla out of its code space. This rotation can be detected using a destructive phase measurement of the ancilla with a resolution set by the ancilla's embedded phase uncertainty $\Delta_{M}(\theta)$ relative to $2 \pi /(N M)$. For illustration, Fig. 5 shows a $\hat{n} \bmod 4$ measurement using two different ancillae: (a) a coherent state and (b) a two-lobe cat state. These can be interpreted (a)

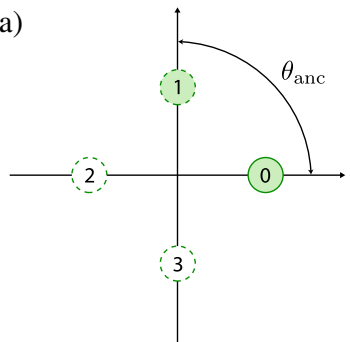

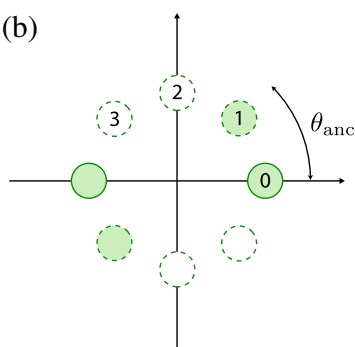

FIG. 5. Measurement of $\hat{n} \bmod 4$ using an ancilla prepared in (a) a coherent state and (b) a two-lobe cat state. Shown are the initial ancilla states (solid circle), and each circle is a potential rotation of the ancilla that depends on the data-rail state being measured. They are labeled by outcomes $\ell$ for a measurement of $\hat{n} \bmod 4$. In both (a) and (b), the specific ancilla rotation shown, $\theta_{\text {anc }}$, corresponds to an $\ell=1$ outcome indicating that the data rail is off the order- $N$ Fock grid. 
(for large enough $\alpha$ ) as a $\left|+_{M}\right\rangle$ code word for $M=1$ and $M=2$, respectively. The motivation for using ancillae with higher $M$ is the fact that errors may occur during the $\hat{C}_{R_{N}}$ gate, as discussed below.

After the measurement, the value of $\ell$ is known, and the data rail is (in the ideal case) projected into an $N$-fold rotation-symmetric subspace: $\hat{\rho} \rightarrow \hat{\Pi}_{N}^{\ell} \hat{\rho} \hat{\Pi}_{N}^{\ell}$, where the projector $\hat{\Pi}_{N}^{\ell}$ is given in Eq. (6). Thus, a $\hat{n} \bmod N$ measurement can be used to prepare code words for an $N$-fold rotation code in the following way: First, prepare the primitive associated with the code, $|\Theta\rangle$, and then measure the excitation number mod $2 N$. Conditional on the ancilla phase being undisturbed $(\ell=0)$, the primitive undergoes the transformation $\hat{\Pi}_{2 N}^{0}|\Theta\rangle \propto\left|0_{N, \Theta}\right\rangle$. Similarly, an ancilla outcome corresponding to $\ell=N$ conditionally prepares $\hat{\Pi}_{2 N}^{N}|\Theta\rangle \propto\left|1_{N, \Theta}\right\rangle$.

\section{A. Error propagation in modular number measurement}

Rotation errors on the ancilla during the $\hat{n} \bmod N$ measurement do not propagate to the data rail (because they commute with the CROT gate) and are not problematic beyond reducing the measurement fidelity. However, any ancilla error that does not commute with $\hat{n}$, such as loss or gain, can induce a rotation error on the data rail (see Sec. VI for a detailed discussion of error propagation). For example, a loss error at a random time during the measurement leads to a rotation error of the data rail by an angle $\theta_{\text {data }} \in[0,2 \pi / N M)$. Thus, using an ancilla with small $M$ brings along the danger of propagating a large rotation error back to the data qubit. In particular, for an $M=1$ ancilla, such as the coherent state in Fig. 5(a), a loss error at an unknown time during the $C_{R_{N}}$ gate completely randomizes the data rail's phase. The maximum magnitude of the rotation error induced by a single ancilla loss or gain event scales as $1 / M$ and, thus, can be reduced by using large- $M$ ancillae. The drawback of using higher- $M$ ancilla is a larger measurement uncertainty in the phase measurement in Eq. (33). This result can be seen in Fig. 5(b) for the two-lobe cat state ancilla.

We note that an improved number-parity syndrome for $N=2$ rotation codes was recently demonstrated experimentally, where the ancilla is encoded in a simple rotation code using three levels of a transmon qubit [31]. In place of a CROT gate, the level-dependent crossKerr interaction $e^{i \pi \hat{n} \otimes(|1\rangle\langle 1|+| 2\rangle\langle 2|)}$ is engineered using sideband drives. The interaction exactly commutes with the dominant ancilla error $|1\rangle\langle 2|$, such that there is no backaction from the ancilla onto the data at all for this error. This syndrome detection scheme can be said to be fault tolerant to a single loss event on the ancilla [31]. Further suppression of state preparation errors can be achieved using a concatenated error-correction scheme, as discussed in Sec. VIII.

\section{ERRORS AND ERROR PROPAGATION}

As was emphasized in the original description of GKP codes in Ref. [3], the discussion of fault tolerance is somewhat different for bosonic codes than for conventional qubit stabilizer codes. For bosonic codes, it is entirely acceptable for every mode involved in the quantum computation to have small errors. In fact, it is unavoidable in practice. In the context of number-phase codes, the "smallness" of errors is relative to a code's number and rotational distance [cf. Eq. (13)], as we explain in more detail below. Therefore, the propagation of small errors is not a major issue. What is catastrophic, however, is if initially small and approximately correctable errors are amplified to the point where the probability of logical errors become likely. Gates that turn small errors into large errors should therefore be avoided.

As we show in the following, the gates introduced in the previous section behave nicely in terms of error amplification. They do not amplify the magnitude of a number-shift error of the form $\sim \hat{a}^{k}\left(\sim \hat{a}^{\dagger k}\right)$, although they might map a number-shift error onto a number-shift error plus a rotation error, $e^{i \theta \hat{n}}$. The upshot is that the new rotation error is proportional to the size of the number-shift error relative to the Fock-space distance, i.e., $\theta \sim k / d_{N}$. In this sense, the new error is proportional in size to the initial error, and, importantly, small errors remain small. Restricting to gates with such well-behaved error propagation properties is likely to be a prerequisite for bosonic codes to have an advantage over unencoded qubits. We return to this point in Sec. VIII, where we discuss a fault-tolerant concatenated quantum computing scheme.

\section{A. Error bases and large vs small errors}

There are two single-mode operator bases that are particularly useful in the context of rotation codes. The first is the set [86]

$$
\left\{\hat{n}^{\ell} \hat{a}^{k},\left(\hat{a}^{\dagger}\right)^{k} \hat{n}^{\ell}\right\}
$$

where $k, \ell \geq 0$ run over all non-negative integers. A straightforward way to show that this set is an operator basis is to first expand the displacement operator in a normal ordered form:

$$
\begin{aligned}
\hat{D}(\alpha) & =e^{\alpha \hat{a}^{\dagger}-\alpha^{*} \hat{a}}=e^{\alpha \hat{a}^{\dagger}} e^{-\alpha^{*} \hat{a}} e^{-(1 / 2)|\alpha|^{2}} \\
& =e^{-(1 / 2)|\alpha|^{2}} \sum_{m, n=0}^{\infty} \frac{\alpha^{m}\left(\alpha^{*}\right)^{n}}{m ! n !}\left(\hat{a}^{\dagger}\right)^{m} \hat{a}^{n} .
\end{aligned}
$$

Since we can write $\left(\hat{a}^{\dagger}\right)^{k} \hat{a}^{k}=\sum_{\ell=0}^{k} c_{k \ell} \hat{n}^{\ell}$ by reordering operators, it follows that $\hat{D}(\alpha)$ can be expanded in terms of the set Eq. (36) [87]. And, since the displacements form an operator basis, so does the set in Eq. (36), and the Kraus operators of any single-mode channel can therefore be expanded in terms of such operators. 
A second useful operator basis is

$$
\left\{e^{i \theta \hat{n}} \hat{a}^{k},\left(\hat{a}^{\dagger}\right)^{k} e^{-i \theta \hat{n}}\right\},
$$

where $k \geq 0$ is an integer and $\theta \in[0,2 \pi)$. That this set is a basis follows from Eq. (36), since $\hat{n}^{\ell}=$ $\int_{0}^{2 \pi}(d \theta / 2 \pi) c_{\ell}(\theta) e^{i \theta \hat{n}}, \quad$ where $\quad c_{\ell}(\theta)=\sum_{m=0}^{\infty} m^{\ell} e^{-i m \theta}$ [88]. We make use of this basis heavily in the following, and it is therefore convenient to introduce a shorthand notation:

$$
\hat{E}_{k}(\theta):= \begin{cases}e^{i \theta \hat{n}} \hat{a}^{|k|} & \text { for } k<0, \\ \left(\hat{a}^{\dagger}\right)^{|k|} e^{i \theta \hat{n}} & \text { for } k \geq 0 .\end{cases}
$$

A negative $k$ thus denotes a downward shift in number and a positive $k$ an upward shift.

An error $\hat{E}_{k}(\theta)$ with $0<|k|<N$ is detectable for any rotation code, which follows from the Fock-space separation of $N$ between $\left|0_{N}\right\rangle$ and $\left|1_{N}\right\rangle$. In general, we cannot make a similarly sharp distinction between detectable and undetectable rotations, but for the ideal number-phase codes introduced in Sec. III, a pure rotation error $E_{0}(\theta)$ with $0<\theta<\pi / N$ is formally detectable.

Intuitively, an error with a small $|k|$ compared to $d_{n} / 2=$ $N / 2$ and small $|\theta|$ compared to $d_{\theta} / 2=\pi /(2 N)$ is a small error and should be correctable to a good approximation for a number-phase code with $N$-fold rotation symmetry. Typically, the codes discussed in this paper are only approximate error-correcting codes for physically relevant noise channels [47].

\section{B. Error propagation}

To show that our gates do not amplify errors too badly and to introduce the error-correction schemes in the next section, we need to understand how the errors propagate through the gates introduced in Sec. IV. Here, we describe errors using $\hat{E}_{k}(\theta)$ given in Eq. (39). The $\left\{\hat{S}_{N}, \mathrm{CROT}_{N M}\right\}$ gates are generated by quadratic powers of the number operator, and, although they are non-Gaussian, we show that they do not amplify errors too badly. In contrast, the gate $\hat{T}_{N}=\exp \left(i \pi \hat{n}^{4} / 4 N^{4}\right)$ is highly nonlinear, and, as we show below, this gate amplifies errors in a more damaging way, which is why we do not rely on using this gate in the scheme introduced in Sec. IV.

All of the unitary gates in our scheme commute with pure rotation errors $e^{i \theta \hat{n}}$. For number-shift errors, we can use that the commutation relation of an operator $e^{c \hat{n}^{\ell}}$ with a power of $\hat{a}$ is given by the general formula

$$
e^{c \hat{n}^{\ell}} \hat{a}^{k}=e^{c\left[n^{\ell}-(n+k)^{\ell}\right]} \hat{a}^{k} e^{c \hat{n}^{\ell}}
$$

A similar commutation relation for $\left(\hat{a}^{\dagger}\right)^{k}$ follows straightforwardly by Hermitian conjugation. We use Eq. (40) to determine how each of the unitary gates in our set amplifies a general error $\hat{E}_{k}(\theta)$; a summary is given in Fig. 6 .

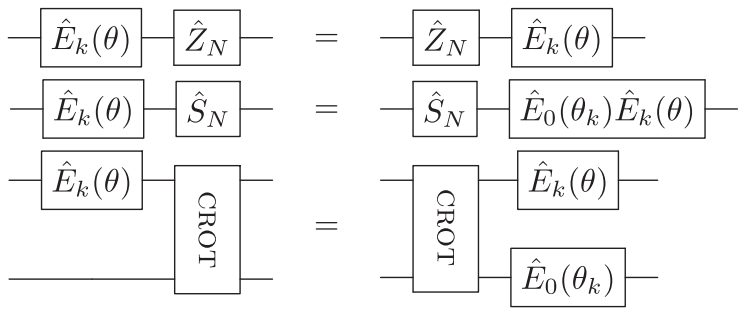

FIG. 6. Propagation of errors through gates. An error $\hat{E}_{k}(\theta)$ at the input of a gate (left column) is propagated to new errors at the output (right column). The circuit identities hold up to an overall phase factor (see the text for the exact relations). The new error $\hat{E}_{0}\left(\theta_{k}\right)$ for $\hat{S}_{N}$ and CROT is a pure rotation error with $\theta_{k}=(\pi k / N M)$, with $N=M$ for $\hat{S}_{N}$.

For gates with $\ell=1$, such as $\hat{Z}_{N}$ in Eq. (2), the prefactor in Eq. (40) is a global phase. Explicitly,

$$
\hat{Z}_{N} \hat{E}_{k}(\theta)=e^{i(\pi k / N)} \hat{E}_{k}(\theta) \hat{Z}_{N} .
$$

Recall that we are labeling the errors with an index $k<0$ for an error $\sim \hat{a}^{|k|}$ and $k>0$ for an error $\sim\left(\hat{a}^{\dagger}\right)^{|k|}$. We see that propagating an $\hat{E}_{k}(\theta)$ error through the Gaussian $\hat{Z}_{N}$ gate only leads to a phase factor, and the error is not amplified at all.

Gates with $\ell=2$, such as $\hat{S}_{N}$ in Eq. (42), on the other hand, introduce new rotation errors:

$\hat{S}_{N} E_{k}(\theta)= \begin{cases}e^{-i\left(k^{2} \pi / N^{2}\right)} \hat{E}_{k}\left(\theta+\frac{\pi k}{N^{2}}\right) \hat{S}_{N} & \text { for } k<0, \\ e^{i\left(k^{2} \pi / N^{2}\right)} \hat{E}_{k}\left(\theta+\frac{\pi k}{N^{2}}\right) \hat{S}_{N} & \text { for } k \geq 0 .\end{cases}$

The initial rotation error $\theta$ is amplified by an amount proportional to the initial number-shift error $k$. Recall that the angular distance of the code is $d_{\theta}=\pi / N$ and the number distance is $d_{n}=N$. If $|k|<d_{n} / 2$ is a small, approximately correctable number-shift error, then the additional rotation error $\pi|k| / N^{2}<d_{\theta} / 2$ is small as well. Thus, if $k$ and $\theta$ are sufficiently small and approximately correctable, we expect the error after the gate to be approximately correctable as well. This behavior is akin to a gate implemented by a constant depth circuit on a qubit stabilizer code, where an initial error can spread at most within a constant-size light cone [89-91].

Commuting an error through the CROT gate spreads errors between the two modes involved in the gate. Label the two modes $a$ and $b$, where $a$ is encoded in an order- $N$ rotation code and $b$ in an order- $M$ rotation code. Commuting CROT through an error on mode $a$ gives

$$
\operatorname{CROT}_{N M} \hat{E}_{k}^{a}(\theta)=\hat{E}_{k}^{a}(\theta) \hat{E}_{0}^{b}\left(\frac{\pi k}{N M}\right) \operatorname{CROT}_{N M},
$$

where the superscript $a / b$ indicates the mode that an operator acts on. Here, the initial number-shift error on mode $a$ spreads to a rotation error on mode $b$. If $|k|<N / 2$ is small with respect to the number distance of mode $a$, 
mode $b$ is rotated by an angle $\pi|k| /(N M)<\pi /(2 M)$, which is small compared to its angular distance. In other words, even though the error has spread to a new rotation error on mode $b$, the error remains small relative to its rotational distance. In the context of $[n, 1 \rrbracket$ qubit codes with a single logical qubit encoded in $n$ physical qubits [92], the CROT gate is analogous to a logical $\bar{C}_{Z}$ gate enacted transversally between two code blocks. How errors propagate through the gate set $\left\{\hat{Z}_{N}, \hat{S}_{N}, \mathrm{CROT}_{N M}\right\}$ is summarized in Fig. 6.

Finally, we show that gates generated by $\hat{n}^{\ell}$ for $\ell>2$, such as the $\hat{T}_{N}$ gate in Eq. (44), are unlikely to be useful for fault-tolerant quantum computing, because errors are amplified and spread in a potentially damaging way. Propagating an error through $\hat{T}_{N}$ produces additional linear and nonlinear rotation errors,

$\hat{T}_{N} E_{k}(\theta)= \begin{cases}\hat{F}_{N}^{k} \hat{E}_{k}\left(\theta+\frac{k^{3} \pi}{N^{4}}\right) \hat{T}_{N} & \text { for } k<0, \\ \hat{E}_{k}\left(\theta+\frac{k^{3} \pi}{N^{4}}\right)\left(\hat{F}_{N}^{k}\right)^{\dagger} \hat{T}_{N} & \text { for } k \geq 0,\end{cases}$

where $\hat{F}_{N}^{k}:=e^{-i\left(k^{4} \pi / 4 N^{4}\right)} e^{-i\left(\pi / 4 N^{4}\right)\left(4|k| \hat{n}^{3}+6 k^{2} \hat{n}^{2}\right)}$ is a nonlinear rotation error. The precise consequences of such nonlinear errors on relevant codes require further study; however, we expect it to be rather damaging — see, e.g., Ref. [93]. As already discussed in Sec. IV, we do not use this gate in our scheme, instead relying on gate teleportation for a logical $\bar{T}$.

\section{ERROR CORRECTION}

Number-phase codes, introduced in Sec. III, are naturally robust to shifts in number with higher- $N$ codes tolerating larger shifts. On the other hand, their robustness to phase errors is directly related to the rotational distance $d_{\theta}=\pi / N$, and rotations reasonably small compared to $d_{\theta} / 2$ can be corrected. Here, we introduce a constructive and practical error-correction scheme for number-phase codes and study its performance numerically.

In a well-designed error-correction scheme, one needs to (i) use gates that do not amplify errors such that they become uncorrectable, (ii) use measurements that are robust to noise, and (iii) carefully construct ancilla interactions such that errors do not spread from ancilla qubits to data qubits in a catastrophic way. In the context of $[n, k]$ qubit stabilizer codes, where $k$ logical qubits are encoded in $n$ physical qubits, two common approaches to faulttolerant error correction are Steane (Steane-EC) and Knill (Knill-EC) error correction [92].

In an adaptation of these schemes to single-mode codes (i.e., codes with each logical qubit encoded in a single bosonic mode), $\llbracket n, 1]$ code blocks are replaced by bosonic modes, and gates and measurement are replaced by faulttolerant bosonic counterparts. For example, in Ref. [3], Steane-EC is adapted to GKP codes by replacing transversal
CNOTs by the fault-tolerant (linear) SUM gate and logical $\bar{X}$ and $\bar{Z}$ measurements by quadrature measurements.

Rotation codes, on the other hand, do not, in general, have a fault-tolerant CNOT (or Hadamard), due to the highly nonlinear nature of the $\bar{X}$ operator. Steane-EC can therefore not be used directly. We get around this issue by adapting a version of Knill-EC to number-phase codes. This approach turns out to have many salient features. As an alternative, we also present a hybrid Steane-Knill scheme in Appendix E.

\section{A. Error correction by teleportation}

We present a bosonic version of Knill-EC [33,94,95] for number-phase codes. Its implementation requires CROT gates, phase measurements, and preparation of $\left|+_{N}\right\rangle$ states, as illustrated in Fig. 7(a). The measurements attempt to distinguish between damaged logical code words in the dual basis [96]. Similar schemes have been considered previously in the context of measurement-based quantum computing [95], where it is occasionally referred to as telecorrection due to the teleportation-based errorcorrection circuit: The circuit in Fig. 7(a) can be recognized as two consecutive one-bit teleportations.

A particular feature of the teleportation-based errorcorrection scheme makes it desirable for number-phase codes. Natural errors for bosonic modes include loss and gain errors that shift the code words off the Fock grid $|k N\rangle$. Actively correcting such errors generally requires difficult, nonlinear shift operations up or down the Fock ladder; see, e.g., Ref. [97]. In contrast, for the circuit in Fig. 7(a), the

(a)
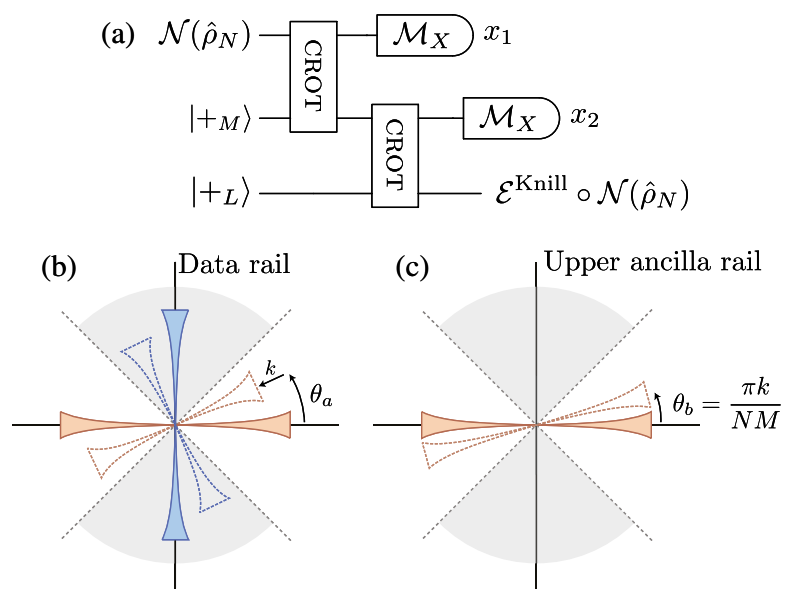

FIG. 7. (a) Schematic illustration of telecorrection using number-phase codes, where $\mathcal{M}_{X}$ are phase measurements with outcomes $x_{i}$. The scheme is based on how errors spread through the CROT gate. An arbitrary error $\hat{E}_{k}\left(\theta_{a}\right)$ on the order- $N$ data rail (b) induces a rotation of the order- $M$ upper ancilla rail by $\theta_{b}=$ $(\pi k / N M)(\mathrm{c})$. Phase measurements on the data rail and the upper ancilla rail extract information about $\theta_{a}$ and $\theta_{b} \sim k$. By the end of the circuit, the logical information is teleported to the bottom order- $L$ ancilla rail (typically, $L=N$ ). 
damaged state is teleported into a fresh ancilla, thus restoring the code space, with only a logical error channel remaining (assuming error-free ancillae). Whether one can correct this remaining logical error depends on the magnitude of the initial error and the error-correcting properties of the code.

The ability to correct errors using teleportation is based on the following circuit identity (cf. Fig. 6):

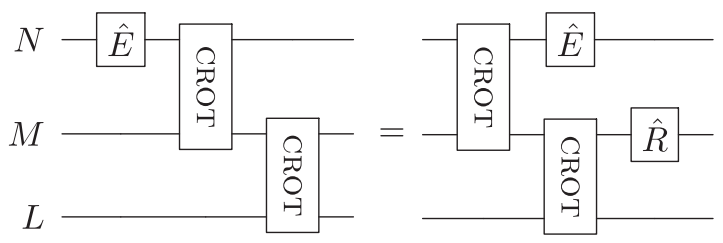

where the labels $N, M$, and $L$ indicate the order of rotation symmetry for each encoded rail, $\hat{E}=\hat{E}_{k}(\theta)$ is an arbitrary error as defined in Eq. (39), and $\hat{R}=\hat{E}_{0}(\pi k / N M)$ is a pure rotation error. Crucially, although the initial error $\hat{E}$ on the top rail spreads to a rotation error $\hat{R}$ on the second rail, it does not spread to an error on the third rail because $\hat{R}$ commutes with СROT. By measuring the first and the second rail, we can teleport the state from the first to the third rail [cf. Eq. (27)] and remove the error in the process. The teleportation is successful as long as one is able to correctly distinguish between code words in the dual basis in the presence of the errors.

We consider the action of the error-correction circuit in more detail for error-free ancillae. Consider an encoded logical state $\hat{\rho}_{N}=\sum_{i, j=0}^{1} \rho_{i j}\left|i_{N}\right\rangle\left\langle j_{N}\right|$ that is sent through an arbitrary single-mode noise channel $\mathcal{N}\left(\hat{\rho}_{N}\right)$ followed by the error-correction circuit in Fig. 7(a). One can show that the corresponding quantum channel can be written

$$
\mathcal{E}^{\mathrm{Knill}} \circ \mathcal{N}\left(\hat{\rho}_{N}\right)=\frac{1}{4} \sum_{i, j=0}^{3} \sum_{\vec{x}} c_{i j}(\vec{x}) \bar{P}_{i} \hat{\rho}_{L} \bar{P}_{j}^{\dagger},
$$

where $\hat{\rho}_{L}=\sum_{i, j=0}^{1} \rho_{i j}\left|i_{L}\right\rangle\left\langle j_{L}\right|$ on the right-hand side represents the same logical state as $\hat{\rho}_{N}$ on the left-hand side but now encoded in the order- $L$ number-phase code of the bottom ancilla rail (in general, this code can be different). The operators $\bar{P}_{i} \in\{\bar{I}, \bar{Z}, \bar{X}, \bar{X} \bar{Z}\}$ are logical Paulis acting on the encoded order- $L$ output state.

The weights $c_{i j}(\vec{x}):=\operatorname{tr}\left[\hat{M}_{x_{1}} \otimes \hat{M}_{x_{2}} \hat{\sigma}_{i j}\right]$ in Eq. (46) are set by the measurement scheme and the noise channel: Here, $\hat{M}_{x_{i}}$ are POVM elements for the two measurements in Fig. 7(a), and the sum over outcomes, $\vec{x}=\left(x_{1}, x_{2}\right)$, should be understood as an integral for continuous measurement outcomes. We also introduce $\hat{\sigma}_{i j}:=\mathcal{U}_{\text {CROT }} \circ \mathcal{N} \circ \mathcal{U}_{\text {CROT }}^{\dagger}(|i\rangle\langle j|)$ with $|i\rangle:=\bar{H}\left|a_{N}\right\rangle \otimes \bar{H}\left|b_{M}\right\rangle$ with $a b$ being the binary representation of $i$ (i.e., $|i\rangle$ runs over $\left|k_{N}\right\rangle \otimes\left|\ell_{M}\right\rangle$ with $k, \ell= \pm$ ). Here, $\mathcal{U}_{\mathrm{CROT}^{\bullet}:=}$ $\mathrm{CROT} \cdot \mathrm{CROT}^{\dagger}$ and $\mathcal{U}_{\mathrm{CROT}}^{\dagger}:=\mathrm{CROT}^{\dagger} \cdot \mathrm{CROT}$. The operator $\hat{\sigma}_{i j}$ thus represents a damaged two-mode logical dual basis, where the noise channel $\mathcal{N}$ is commuted through the CROT gate.

Crucially, Eq. (46) shows that after error correction the remaining error channel acts entirely in the logical subspace of the output mode, for any noise channel $\mathcal{N}$ acting on the input mode. In principle, a measurement-dependent Pauli recovery $\bar{P}_{x}$ could be applied, but the simplest solution is to track the most likely Pauli correction $\bar{P}_{i *}$ in a Pauli frame. The right Pauli correction can be guessed using, for example, a maximum likelihood decoder

$$
i^{*}(\vec{x})=\operatorname{argmax}_{i} \operatorname{tr}\left[\hat{M}_{x_{1}} \otimes \hat{M}_{x_{2}} \hat{\sigma}_{i i}\right] .
$$

\section{B. Numerical results for loss and dephasing with error-free ancillae}

In a recent work, Albert et al. [44] investigated the errorcorrection performance of several bosonic codes including cat and binomial under pure loss. In that work, a recovery map was found by numerical optimization, and it was shown that bosonic codes can heavily suppress loss errors, in principle. While it sets a useful bound, numerical optimization does not provide any information about how to implement the recovery map in practice. An important step forward is therefore to compare an explicit error-correction scheme to the numerically optimal recovery map for relevant noise channels. To make this comparison, we focus in this section on a setting with noise-free ancillae and idealized measurements. Dealing with faulty ancillae and measurement noise is a nontrivial problem which we return to in a qualitative discussion in Sec. VIII. One of our main results is that the teleportation-based errorcorrection scheme presented above is close to optimal in the relevant region of small noise, with a gap from the optimal scheme arising primarily from inherent noise in the phase measurements.

In this section, we numerically compute the average gate fidelity [98] for a channel composed of an ideal encoding of a qubit into a number-phase code, followed by a singlemode noise channel, and finally error correction using noise-free ancillae and ideal gates [99]. We have confirmed that the Knill-EC scheme from the previous section and the hybrid Steane-Knill scheme presented in Appendix E perform identically for the codes and parameter ranges we investigate.

In the idealized situation where the ancillae are noisefree, one can use, e.g., a simple $M=1$ cat code with $\left|0_{\text {cat }}\right\rangle \propto|\alpha\rangle+|-\alpha\rangle,\left|1_{\text {cat }}\right\rangle \propto|\alpha\rangle-|-\alpha\rangle$ for the middle ancilla rail in Fig. 7(a). For large enough $\alpha$, rotations of the state $\left|+_{\text {cat }}\right\rangle$ can be detected arbitrarily well with phase measurements, giving essentially perfect syndrome measurements for this rail. Moreover, we set $L=1$ for the bottom (output) rail and use the trivial encoding, $\left|0_{\text {triv }}\right\rangle=|0\rangle$ and $\left|1_{\text {triv }}\right\rangle=|1\rangle$, for this mode. The 

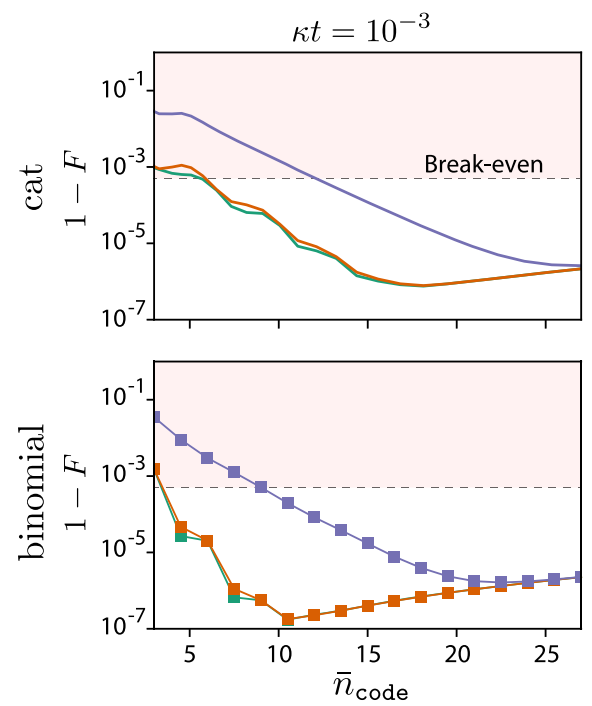
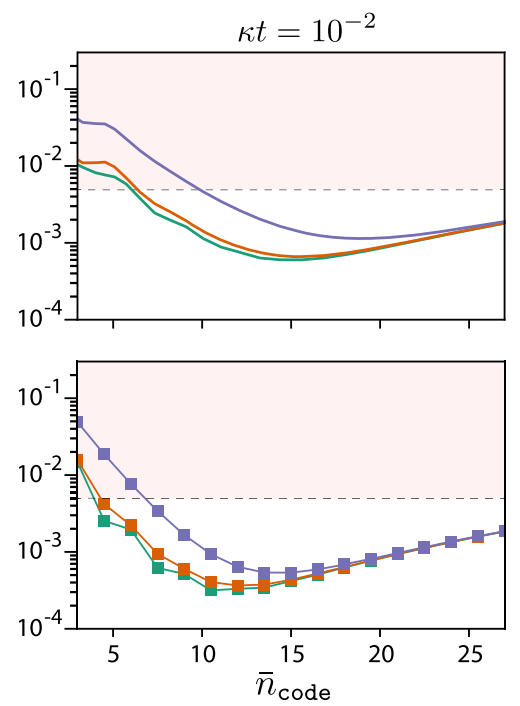
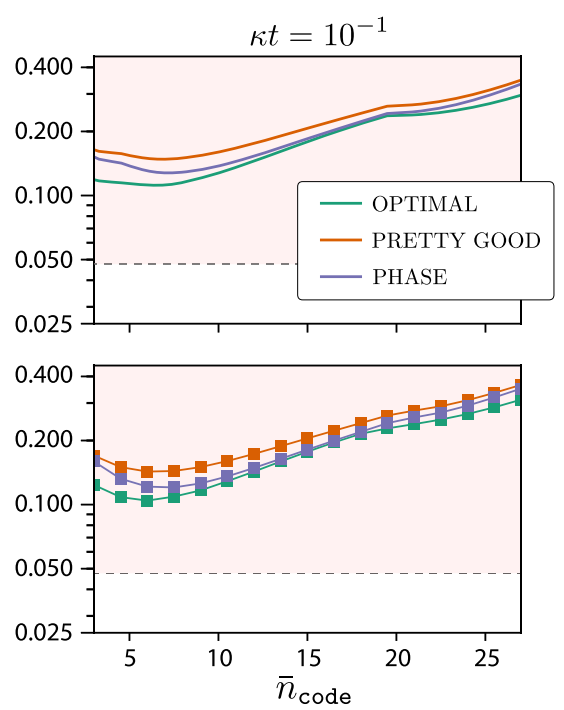

FIG. 8. Average gate infidelity as a function of the average excitation number in the code, $\bar{n}_{\text {code }}$ [Eq. (11)], for an $N=3$ cat code (top row) and binomial code (bottom row). We compare the theoretically optimal error-correction scheme found numerically (OPTIMAL) to our telecorrection scheme using pretty good measurements (PRETTY GOOD) and canonical phase measurements (PHASE). The encoded data rail is subject to noise through evolution under the master equation in Eq. (49) with equal loss and dephasing strength, $\kappa_{\phi} t=\kappa t$. Each column shows results for a different amount of total noise $\kappa t$ before error correction is performed. A code performs better than break even (the uncorrected trivial encoding) whenever the gate infidelity is below the dashed line (falls outside the shaded region).

error-correction circuit therefore also decodes the encoded information.

The total quantum channel we consider is

$$
\mathcal{E}^{\text {Knill }} \circ \mathcal{N} \circ \mathcal{S}
$$

where $\mathcal{S} \bullet:=\hat{S} \bullet \hat{S}^{\dagger}$, with $\hat{S}:=\left|0_{N}\right\rangle\left\langle 0|+| 1_{N}\right\rangle\langle 1|$, is the ideal encoding map for a given code, $\mathcal{N}$ is a noise map, and $\mathcal{E}^{\text {Knill }}$ is the error-correction circuit. This is a logical channel with qubit input and qubit output, due to the use of a trivial encoding for the final output mode of the error-correction circuit. Note that $\mathcal{E}^{\text {Knill }} \circ \mathcal{N}$ is given by Eq. (46) in the case when recoveries are tracked in software rather than applied explicitly. To compute the average gate fidelity, we explicitly apply a correction $\bar{P}_{i^{*}}^{\dagger}$ to undo the most likely Pauli error using the decoder in Eq. (47).

The noise channel we consider consists of simultaneous loss and dephasing. Specifically, $\mathcal{N}(\hat{\rho})$ is the solution to the master equation

$$
\dot{\hat{\rho}}=\kappa \mathcal{D}[\hat{a}] \hat{\rho}+\kappa_{\phi} \mathcal{D}[\hat{n}] \hat{\rho},
$$

integrated up to some unitless time $\kappa t$, with $\mathcal{D}[\hat{L}] \hat{\rho}:=$ $\hat{L} \hat{\rho} \hat{L}^{\dagger}-\frac{1}{2} \hat{L}^{\dagger} \hat{L} \hat{\rho}-\frac{1}{2} \hat{\rho} \hat{L}^{\dagger} \hat{L}$. A Kraus-operator decomposition of $\mathcal{N}$ is given in Appendix $F$.

We wish to quantify the performance of the telecorrection scheme from Sec. VII A. To this end, we compare the average gate fidelity for the channel in Eq. (48) with a channel where the error-correction map is replaced by the completely positive trace-preserving map $\mathcal{E}^{\text {opt }}$ that maximizes the fidelity. The latter can be found by solving a semidefinite program [44], and we refer to this recovery as OPTIMAL. The discrepancy between the teleportationbased scheme and the optimal is due in large part to the inherent uncertainty of the phase measurementscf. Sec. II C 2 -and is especially prominent for small average excitation numbers. We obtain further insight into the origin of this gap by comparing canonical phase measurements to "pretty good measurements" (see Appendix D) for the data rail in the circuit [Fig. 7(a)]. The measurement on the middle ancilla rail is in both cases a canonical phase measurement as defined in Eq. (14) [100]. We refer to these two error-correction schemes as PHASE and PRETTY GOOD, respectively, depending on which measurement is performed on the data rail. We emphasize that the PRETTY GOOD scheme is used in our numerics purely to gain insight into the origin of the gap between PHASE and OPTIMAL.

We focus here on cat and binomial codes as examples of number-phase codes. As expected, these codes have a similar performance for large average excitation number $\bar{n}_{\text {code }}$ [Eq. (11)] but can show significant differences for smaller $\bar{n}_{\text {code }}$ [48]. Remarkably, we find that the PHASE error-correction scheme approaches OPTIMAL for large $\bar{n}_{\text {code }}$ and small noise strength and that the PRETTY GOOD scheme is near OPTIMAL for almost all $\bar{n}_{\text {code }}$ and small noise strengths.

In Fig. 8, we show examples of the average gate infidelity $1-F[98,101]$ for an $N=3$ cat code (top row) and $N=3$ binomial code (bottom row) as a function of $\bar{n}_{\text {code. }}$. We fix the dephasing rate to be equal to the loss 

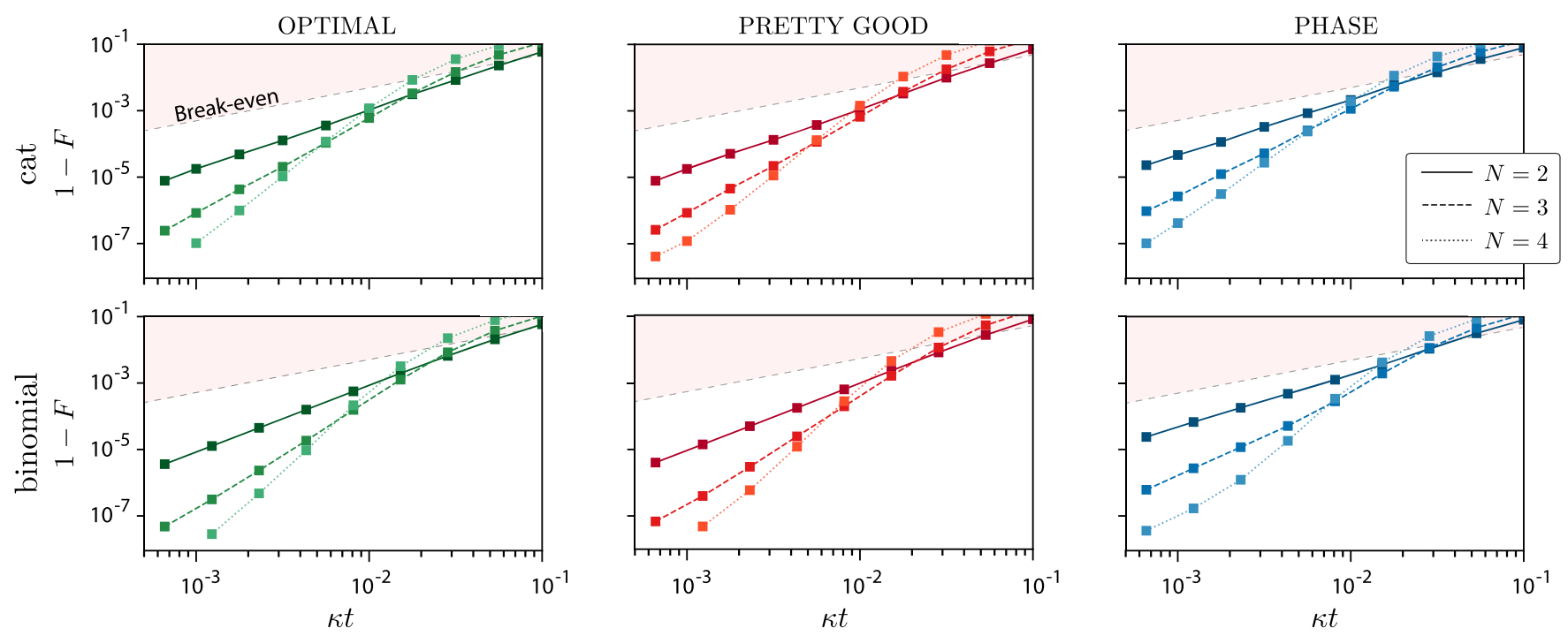

FIG. 9. Average gate infidelity as a function of noise strength $\kappa t$ for cat codes (top row) and binomial codes (bottom row). For each $N$ and each $\kappa_{\phi} t=\kappa t$, the optimal average excitation number $\bar{n}_{\text {code }}$ is used for each code. A code performs better than break even whenever the gate infidelity is below the dashed line (outside the shaded region). We show only results for $1-F \geq 2.5 \times 10^{-8}, \kappa t \geq 0.5 \times 10^{-3}$, and $N \leq 4$ due to the prohibitively large Fock-space truncation needed for numerical simulations and numerical accuracy issues for very small infidelity.

rate $\kappa_{\phi}=\kappa$ and compare three different noise strengths parameterized by $\kappa t$. The dephasing rate $\kappa_{\phi}$ models both natural dephasing and additional uncertainty in the phase measurements, which motivates choosing a fairly large $\kappa_{\phi}$. The dashed line in the figures shows the average gate infidelity using the trivial encoding $\left|0_{\text {triv }}\right\rangle=|0\rangle$ and $\left|1_{\text {triv }}\right\rangle=|1\rangle$ on the data rail with no error correction. This line marks the break-even point above which, in the pink region, encoding and error correction perform worse and provide no advantage.

There are several takeaway messages from the results in Fig. 8. First, as advertised, both the PRETTY GOOD and PHASE error-correction schemes are near optimal for large $\bar{n}_{\text {code }}$. While for PHASE there is a significant gap at small to moderate $\bar{n}_{\text {code }}$, the PRETTY GOOD scheme performs very close to optimal except for very small $\bar{n}_{\text {code }}$ and/or large noise strengths. Second, for all schemes, the codes exhibit an optimal $\bar{n}_{\text {code }}$ where the infidelity reaches a minimum. Under small loss rates, the optimal $\bar{n}_{\text {code }}$ for PHASE is much larger than for PRETTY GOOD and OPTIMAL, due to the poor ability of phase measurements to distinguish states with small $\bar{n}_{\text {code }}$. Third, we see that the binomial codes generally outperform cat codes for small $\bar{n}_{\text {code }}$ and low noise strength, while the performance is identical for large $\bar{n}_{\text {code }}$. We note that, for smaller dephasing rates, binomial codes have an increasing advantage over cat codes, since a more loss-dominated noise channel generically favors smaller $\bar{n}_{\text {code }}$ [44].

Figure 8 also shows that there is a significant potential for going beyond break even, at least within the idealized error model considered here. To investigate this potential further, we compare cat and binomial codes with different
$N$ in Fig. 9. For each $N$ and each code, we choose the optimal $\bar{n}_{\text {code }}$. We then plot the logical infidelity $1-F$ as a function of the noise strength for the three different errorcorrection schemes OPTIMAL, PRETTY GOOD, and PHASE.

There are two main observations from Fig. 9. First, both cat and binomial codes break even by orders of magnitude for all error-correction schemes under the simplified noise model considered here. We can identify break-even pseudothresholds, defined as the noise strength $(\kappa t)_{\text {be }}$, where the infidelity is equal to that of the trivial encoding with no error correction (i.e., where the lines in Fig. 9 cross the boundary between the pink and the white region). The break-even thresholds are fairly high, falling in the 1\%-10\% range, for the range of $N$ considered, but they decrease with larger $N$. The second observation is that the logical infidelity $1-F$ falls off more rapidly with higher $N$, over a range of $\kappa t$. However, the gain in performance is diminishing with increasing $N$. We do not expect the infidelity to become arbitrarily small with increasing $N$, since the protection against phase noise decreases. The relatively large reductions in infidelity with increasing $N$ seen in Fig. 9 suggest that the performance is not limited by dephasing for the noise parameters and codes considered here.

The results presented in this section have consequences for the prospects of using number-phase codes for faulttolerant quantum computing. On one hand, the large breakeven potential motivates further study of these codes under realistic noise models including noisy ancillae, noisy encoding, more realistic measurement models, and noise during gates. A pertinent question is how sensitive the performance is to nonlinear unitary errors such as those 
from unwanted self-Kerr interactions. On the other hand, the expectation that arbitrarily low infidelities cannot be reached means that cat and binomial codes must ultimately be concatenated with a second code to suppress errors further.

\section{ROAD MAP TO FAULT TOLERANCE}

In this section, we outline a scheme that achieves a longstanding goal for number-phase codes such as cat and binomial: a fault-tolerant universal set of operations. First, let us summarize the challenges that need to be overcome for fault tolerance with number-phase codes (and rotation codes more generally).

(i) We touch upon some of the challenges with state preparation in Sec. IV B. A modular excitationnumber measurement (Sec. V) can be performed to check a state for loss and gain errors but not without the risk of introducing new rotation errors. Unfortunately, we have not been able to find a practical, nondestructive measurement to check for rotation errors. If only number-shift errors are checked for, state-preparation noise is biased toward rotation errors [102].

(ii) Realistic syndrome measurements are going to be rather noisy. A standard approach, especially in topological stabilizer codes, is to repeat the syndrome measurements multiple times and decode based on a record of measurement outcomes [77]. However, due to the destructive nature of the measurements in Fig. 7, they cannot straightforwardly be repeated.

(iii) We expect that logical error rates can be suppressed only up to a point for realistic error channels. In particular, there is a trade-off between resilience to number-shift and rotation errors; cf. Eq. (13) [103].

One way to overcome these issues is to concatenate the bosonic code with a conventional qubit code so that the qubit code can deal with errors not handled by the bosonic code, which is broadly the approach taken recently for GKP codes, e.g., in Refs. $[11,13,14]$. Tailoring fault-tolerant gadgets to the respective strengths and weaknesses of the bosonic code is, however, not a trivial task. If this process is not done carefully, the advantage of using a bosonic code at the ground level is diminished, and any performance benefits over using bare qubits might be lost. Simply, we want to optimize the concatenated scheme to maximally exploit the error-correction properties and noise resilience of the ground-level bosonic code.

In the following, we outline how this goal can be achieved using concatenation with a Bacon-Shor subsystem code $[34,35,104]$. This specific choice serves to illustrate the broader point of how a fault-tolerant concatenated scheme can be tailored to exploit the strengths of the underlying bosonic code.
A single Bacon-Shor code can have a high fault-tolerant pseudothreshold at an intermediate code size, even though the threshold vanishes in the limit of an infinitely large system size $[37,105,106]$. Bacon-Shor codes are attractive candidates for near- and intermediate-term logical qubits due to their geometrically local layout in two dimensions and weight-two gauge operator checks $[37,104,107]$. Specifically, we make use of a teleportation-based scheme developed by Aliferis and Preskill for repetition codes in Ref. [36] and generalized to Bacon-Shor codes by Brooks and Preskill in Ref. [37]. We refer to these two works collectively as the Aliferis-Brooks-Preskill (ABP) scheme.

The key to our fault-tolerant gadgets is to use only bosonic operations that do not amplify errors within a mode and do not spread small errors to larger errors at the bosonic level. The error-correction properties of the bosonic code can then be exploited fully. The bosonic code operations we make use of are

$$
\mathcal{G}_{\text {bosonic }}=\left\{\mathcal{P}_{\left|+_{N}\right\rangle}, \mathcal{M}_{X}, \text { CROT }\right\} .
$$

We also assume universal control over two-level ancillae or, equivalently, bosonic modes in the trivial encoding $\left|0_{\text {triv }}\right\rangle=|0\rangle,\left|1_{\text {triv }}\right\rangle=|1\rangle$, and $\mathrm{CROT}_{N 1}$ gates between the two-level ancillae and order- $N$ rotation codes. Using the $\mathrm{ABP}$ scheme, this assumption together with the operations in Eq. (50) suffice for universal quantum computing. Remarkably, the bosonic error correction is seamlessly integrated into the Bacon-Shor error correction with our approach, such that minimal overhead is incurred. The bosonic error correction is in this sense highly efficient.

\section{A. Parallel teleportation}

Let us start with the issue of measurement errors in the error-correction scheme in Fig. 7, as an entry point into the ABP scheme. As already stated, because the syndrome information is extracted using destructive measurements, the measurements cannot simply be repeated. However, since the measurements and state preparation in Fig. 7 are all in the $\bar{X}$ basis, a simple remedy is to concatenate with a length- $n$ repetition code (for $n$ odd) in the dual basis:

$$
\left| \pm_{\mathcal{L}}\right\rangle:=\left| \pm_{N}\right\rangle^{\otimes n}
$$

Logical $\hat{X}_{\mathcal{L}}$ measurements and state preparation for the repetition code are then simply independent phase measurements and independent preparation of the $n$ bosonic modes using a number-phase encoding. Measurement errors are suppressed by performing a majority vote on the $n$ outcomes [108]. This fact means that, if we can generalize the teleportation circuit in Fig. 7 to teleportations of repetition code blocks, we can use the simple encoding in Eq. (51) to suppress measurement errors. Equation (51) is essentially a classical encoding, and the repetition code 
plays a role analogous to repeated measurements in topological codes [77].

A useful observation is that a $\hat{C}_{Z}$ gate enacted between each qubit in a repetition code block and a single ancilla qubit acts as a logical $\hat{C}_{Z}$ between the repetition code block and the one ancilla. So, for example, the red CROT gates between the upper block and the ancilla in Fig. 10(a) gives a logical $\hat{C}_{Z}$ between this block and the ancilla, leading to the identity with Fig. 10(b), where the thick lines represent repetition code blocks of length $n$. The circuit in Figs. 10(a) and 10(b) represents a nondestructive measurement of $\hat{Z}_{\mathcal{L}} \otimes \hat{Z}_{\mathcal{L}}$, where $\hat{Z}_{\mathcal{L}}$ is a logical Pauli- $Z$ for the repetition code block. To increase robustness to ancilla measurement errors, we can repeat this circuit $r$ times, as illustrated in Figs. 10(c) and 10(d) to get a robust $\mathcal{M}_{Z_{\mathcal{L}} Z_{\mathcal{L}}}$ gadget. Error correction is performed, following Ref. [36], by the logical one-bit teleportation circuit in Fig. 10(e). Note that, with $n=r=1$, the error-correction gadget in Fig. 10(e) is identical to that in Fig. 7(a). The scheme outlined in Fig. 10 is thus a direct generalization of the scheme we introduced above in Sec. VII, and the error-correction circuit performs both bosonic-code and repetition-code error correction in one step.

Let us pause at this point to clarify what is achieved by concatenating with a repetition code. First of all, the repetition code is used to suppress measurement errors, but it also protects against rotation errors more generally. (a)

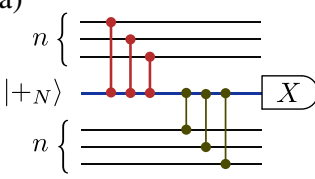

(c)

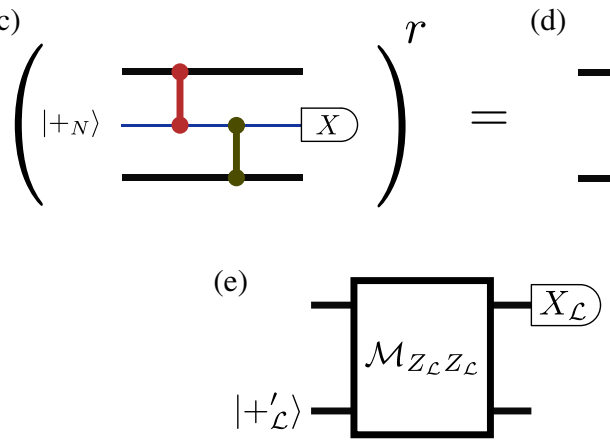

(b)

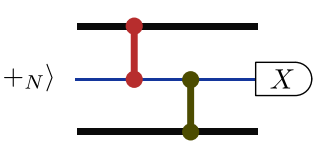

d)

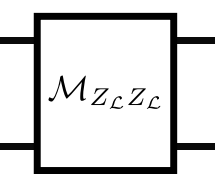

FIG. 10. Building blocks of the error-correction scheme from Ref. [36]. (a) The wires represent number-phase codes, and the $\hat{C}_{Z}$ gates are CROT gates. A $\hat{C}_{Z}^{\otimes n}$ gate enacted between a repetition code block and a single ancilla acts as a logical $\hat{C}_{Z}$. (b) The same circuit as in (a), where the thick wires represent length- $n$ repetition code blocks. (c),(d) By repeating a nondestructive measurement of $\hat{Z}_{\mathcal{L}} \otimes \hat{Z}_{\mathcal{L}} r$ times, we get a robust $\mathcal{M}_{Z_{\mathcal{L}} Z_{\mathcal{L}}}$ gadget. (e) The errorcorrection gadget from Ref. [36]. Here, $\left|+_{\mathcal{L}}\right\rangle=\left|+_{N}\right\rangle{ }^{\otimes n}$, and the $\hat{X}_{\mathcal{L}}$ measurement is independent, destructive phase measurement of each mode in the repetition code block.
The latter is especially useful if state-preparation noise is biased toward rotations, as is the case if number-syndrome checks are used in this step.

To further understand the concatenated bosonicrepetition code's robustness to number-shift errors, consider for the sake of concreteness an $N=2$ number-phase code and a noise channel biased toward loss with negligible gain. An $N=2$ number-phase code can on its own be robust against a single loss error when gain is negligible. Say, for example, a single loss error occurs on one of the ancilla rails in the $\mathcal{M}_{\mathcal{Z}_{\mathcal{L}} Z_{\mathcal{L}}}$ gadget in Fig. 10. It will spread to a clockwise rotation error of magnitude $\pi / 4$ on all of the upper and lower data rails. This error-spread is acceptable, because the number-phase code with angular distance $d_{\theta}=$ $\pi / 2$ can deal with rotation errors of this magnitude (note that, under the assumption of no gain, one should bias the decoding procedure in Fig. 3 toward clockwise rotations). Confidence in detecting this loss error is increased by the fact that it is present in all $n$ phase measurements on the upper data rails.

On the other hand if, say, two of the $r$ ancillae in the $\mathcal{M}_{\mathcal{Z}_{\mathcal{L}} Z_{\mathcal{L}}}$ have a single loss error, this error will propagate to a rotation error of magnitude $\pi / 2$, which is too large for an $N=2$ number-phase code to handle. Consequently, this error leads to a logical error at the repetition-code level with high likelihood. The number of ancillae $r$ and the repetition-code length $n$ thus have to be chosen dependent on the order of rotation symmetry of the number-phase code, $N$, as well as the physical loss rate.

We note that concatenation with a repetition code has recently been proposed in the context of $N=1$ cat codes $[109,110]$. An $N=1$ cat code does not provide any protection against loss or gain errors but gives rise to a highly biased effective noise model, because transitions $|\alpha\rangle \rightarrow|-\alpha\rangle$ are exponentially suppressed in $|\alpha|^{2}$. In this situation, no active error correction is performed at the bosonic-code level, and the purpose of the repetition code is to protect against loss and gain errors, which is orthogonal to what we propose here.

\section{B. Cats of cats and repetitions of repetitions}

The above concatenated error-correction scheme can be improved following Ref. [37]. The techniques presented there can be adopted straightforwardly to the present context, so we only briefly comment on the main ideas here.

Despite the scheme already described above being robust to both loss and rotation errors, it may be impractical to prepare number-phase codes with very large $N$. This constraint limits the number of ancilla measurements, as explained above, because a single ancilla loss or gain error spreads to all of the data rails, and vice versa. This problem can be overcome by replacing each of the $r\left|+_{N}\right\rangle$ middlerail ancillae in Fig. 10 by logical $\left|{ }^{\prime}{ }_{\mathcal{L}}\right\rangle$ for a repetition code which is dual to Eq. (51): 


$$
\left| \pm_{\mathcal{L}}^{\prime}\right\rangle:=\frac{1}{\sqrt{2}}\left(\left|0_{N}\right\rangle^{\otimes n} \pm\left|1_{N}\right\rangle^{\otimes n}\right)
$$

The logical Pauli- $X$ for this code is $\hat{X}^{\otimes n}$. However, since we require only destructive measurements of the ancillae, one can perform a destructive phase measurement to measure each of the $n$ ancilla modes in the $\left| \pm_{N}\right\rangle$ basis, as in Fig. 3, and take the parity of the digitized outcomes.

The purpose of using the dual repetition code [Eq. (52)] for the middle-rail ancillae is that a fully transversal $\hat{C}_{Z}^{\otimes n}$ gate enacted between the two codes [Eqs. (51) and (52)] performs a logical $\hat{C}_{Z}$ gate between the two code blocks. The basic building block of the error-correction gadget then simply becomes an encoded version of the scheme from Fig. 7(a), as illustrated in Fig. 11. The error-correction scheme is otherwise identical to that in Fig. 10. The crucial difference is that, since the $\hat{C}_{Z}$ gates are now fully transversal, an error on any mode in the circuit spreads to at most two other modes.

The state $(1 / \sqrt{2})|0\rangle^{\otimes n}+(1 / \sqrt{2})|1\rangle^{\otimes n}$ is sometimes referred to as a "cat state." Since any bosonic rotation code is also a generalized cat code, according to Eq. (3), we can refer to the state $\left|{ }^{\prime}{ }_{\mathcal{L}}\right\rangle$ as a "cat of cats." Of course, preparing these cat states also incurs overhead. As explained in Ref. [37], this preparation can be done faulttolerantly using only the basic operations $\mathcal{G}_{\text {bosonic }}$ (with about $2 n$ CROT gates and $n$ extra ancillae for a length- $n$ cat of cats).

As explained in Ref. [37], one can also use cat of cats $\left|{ }^{\prime}{ }_{\mathcal{L}}\right\rangle$ of any length $1 \leq p \leq n$. For $p<n$, some ancilla modes interact with multiple data modes. The two extremes $p=1$ and $p=n$ are shown in Fig. 10, 11, respectively. In general, both the cat-of-cats length $p$ and the number of repeated measurements $r$ should be optimized to the noise model [37].

The last extension from Ref. [37] is to replace the repetition code with a Bacon-Shor subsystem code. A Bacon-Shor code can be thought of as a concatenation

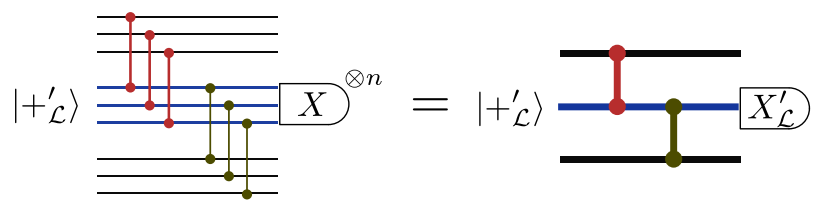

FIG. 11. An extension of the error-correction scheme from Ref. [36] where each ancilla is encoded in the state $\left|+_{\mathcal{L}}\right\rangle$ [Eq. (52)]. On the left, the rails represent number-phase codes, the $\hat{C}_{Z}$ gates are CROT gates, and the measurement is an independent destructive phase measurement of each mode in a block. The parity of the $n$ digitized measurement outcomes is computed to perform a measurement in the $\left| \pm_{\mathcal{L}}{ }^{\prime}\right\rangle$ basis. On the right, the thick lines represent repetition code blocks, and the gates are logical $\hat{C}_{Z}$ between the code blocks. Error correction is otherwise performed exactly as in Fig. 10: The circuit is repeated $r$ times, and, finally, the upper data block is measured $\mathcal{M}_{X}{ }^{\otimes n}$, with the lower data block prepared in $\left|+_{N}\right\rangle^{\otimes n}$. of a length- $n$ repetition code with a second, length- $m$, repetition code. While the code in Eq. (51) gives protection only against rotation errors (beyond the protection provided by the bosonic code), a Bacon-Shor code with $m>1$ provides additional protection against loss and gain errors. In general, one can optimize the ratio $m / n$ to the noise model and the capabilities of the bosonic code at the ground level. Error correction is still performed with a gadget as in Fig. 10(e) using a relatively straightforward generalization of the $\mathcal{M}_{Z_{\mathcal{L}} Z_{\mathcal{L}}}$ measurement and where logical $\left|+_{\mathcal{L}}\right\rangle$ for the bosonic-Bacon-Shor code is now a product of cat-of-cats states [37]. Crucially, the only operations ever needed are CROT gates between pairs of modes, preparation of $\left|+_{N}\right\rangle$ states, and destructive phase measurements to measure each mode independently in the $\left| \pm_{N}\right\rangle$ basis.

\section{Universality}

In the ABP scheme, a logical CNOT gate is built out of the fundamental operations in Eq. (50) [36,37]. Universality is furthermore achieved by injecting noisy states $|+i\rangle=(|0\rangle+i|1\rangle) / \sqrt{2}$ and $|T\rangle=\left(|0\rangle+e^{i \pi / 4}|1\rangle\right) / \sqrt{2}$ directly into the Bacon-Shor (or repetition) code block. In the present context, state injection can be performed by preparing the two lowest levels of an ancilla, e.g., a transmon, in the desired state $|\psi\rangle$ and using a cross-Kerr interaction between the ancilla and a bosonic mode to perform a $\mathrm{CROT}_{N 1}$ gate. The one-bit teleportation gadget in Fig. 10(e) is then used to teleport the ancilla state into a code block. In this case, the upper rail in Fig. 10(e) is the unencoded two-level ancilla in the state $|\psi\rangle$, and the lower rail is the Bacon-Shor code block. This circuit prepares the code block in an encoded state $\left|\psi_{\mathcal{L}}\right\rangle$ up to a known measurement-dependent logical Pauli. Several noisy $\left|+i_{\mathcal{L}}\right\rangle$ or $\left|T_{\mathcal{L}}\right\rangle$ states can be distilled to higher fidelity using standard state distillation protocols, as analyzed in Ref. [37].

As a final remark, we emphasize that, although the fullblown scheme using Bacon-Shor codes and cat-of-cats ancillae is quite complex, the simplest repetition code with single-mode ancillae should already be able to demonstrate all the key ingredients of a fault-tolerant scheme. It is therefore a promising candidate to realize small-scale logical qubits in near-to-medium-term experiments [107].

\section{CONCLUSIONS AND DISCUSSION}

We have presented a broad class of bosonic errorcorrecting codes characterized by discrete rotation symmetry, which we call bosonic rotation codes or simply rotation codes for short. Several well-known codes, including cat, binomial, and $\mathrm{ON}$ codes, are rotation codes. Cat and binomial codes, moreover, belong to a subset of rotation codes, the number-phase codes, which are characterized by vanishing phase uncertainty for large excitation numbers. Another member of this subset is Pegg-Barnett codes, which can be interpreted as the shift-resistant qudit codes 


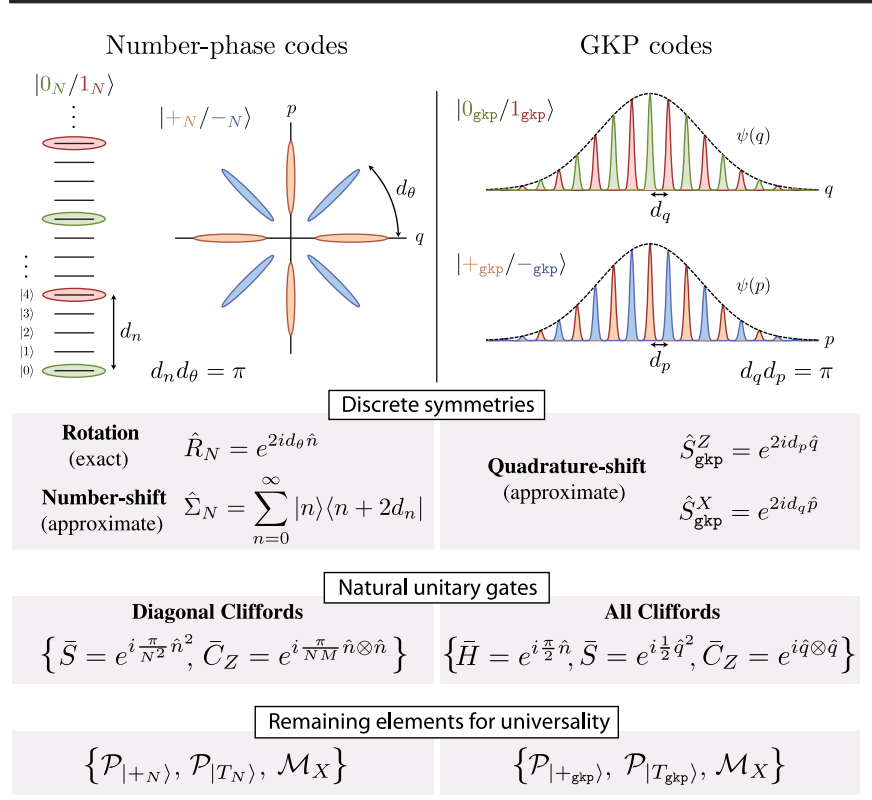

FIG. 12. Comparison between the number-phase codes introduced in this work and GKP codes [3]. For simplicity, we consider the square-lattice GKP code. The cartoon shows code words for an $N=4$ number-phase code, with $d_{n}=4$ and $d_{\theta}=\pi / 4$, and a GKP code, with $d_{q}=d_{p}=\sqrt{\pi}$. For each code type, there are unitary gates that are "natural" in the sense that they are generated by physically natural Hamiltonians and that they map small errors to small errors. To achieve universality, the unitary gates are supplemented with state preparation and Pauli measurements for both code types. Note that it was recently shown that the GKP magic state $\left|T_{\text {gkp }}\right\rangle$ can be distilled using only Gaussian operations [114]. The number-phase-code analogs to the envelopes in the approximate GKP code words are numberamplitude envelopes such as those in Fig. 4.

introduced in Ref. [3] embedded in the infinite-dimensional Hilbert space of a bosonic mode.

The theoretical framework we present here for numberphase codes allows a natural comparison with GKP codes, summarized in Fig. 12. First, number-phase codes are analogous to approximate GKP codes, with the number and phase playing dual roles in place of position and momentum [111]. Second, the fact that the GKP stabilizers are generated by Hamiltonians linear in annihilation and creation operators leads to Gaussian Clifford gates. In contrast, number-phase codes have one symmetry generated by a quadratic Hamiltonian $\left(\hat{R}_{N}\right)$ and one non-Gaussian symmetry $\left(\hat{\Sigma}_{N}\right)$. As a consequence, a subset of the Clifford group - the diagonal Cliffords-can be generated by Hamiltonians that are quartic in annihilation and creation operators, while the full Clifford group contains highly nonlinear gates. The smaller set of natural unitary gates and the higher degree of nonlinearity is a disadvantage compared to GKP codes. However, the issue of nonlinearity is not entirely clear cut. Self- and cross-Kerr interactions are very natural in some platforms $[19,113]$, and some of the GKP Cliffords require underlying physical nonlinearities as well-for example, to realize the shear gate $\bar{S}$ and the two-mode gate $\bar{C}_{Z}$.

A feature of number-phase codes is that phase measurement is a natural candidate for a robust logical measurement in the dual basis. In practice, phase measurements are realized by heterodyne and homodyne measurements as well as adaptive homodyne schemes [67,115]. The existence of a robust dual-basis measurement is crucial for the quantum computing and error-correction schemes presented in Secs. IV and VII.

The entangling gate (СROT) in our scheme is a controlled-rotation gate based on a cross-Kerr interaction between two modes. The CROT gate plays a central role in many pieces of our quantum computing scheme including gate teleportation, modular number measurement, and error correction. An attractive feature of the CROT gate is that it can be used to interface any two rotation codes. In fact, the CROT gate can even be used as an entangling gate between any bosonic rotation code and a square- or hexagonal-lattice GKP code, due to the underlying rotation symmetry of the GKP code lattices; see Appendix C. This fact could be used in hybrid schemes employing multiple encodings and for switching between codes using teleportation. The generality of the CROT gate is similar in spirit to that of the exponential-SWAP (eSWAP) gate [116,117], which is agnostic to code type but makes no guarantees about error propagation. In contrast, the Kerr-based interactions that underlie CROT gates amplify errors only in a very limited way and are, thus, attractive candidates for use in a fault-tolerant scheme.

There are two particularly salient features of the teleportation-based error-correction scheme we introduced in Sec. VII. The first is that, for a large average excitation number in the code, the performance (as measured by average gate fidelity) is near that of the optimal recovery map allowed by the laws of quantum mechanics. For a small average excitation number, the gap from optimal stems mainly from the inability of phase measurements to accurately distinguish the logical code words in this limit. The second is that teleportation-based error correction does away with the need for explicit recovery operations to restore the code space, and logical recoveries can be tracked entirely in software. Since explicit recovery operations would require highly nonlinear implementations, this is a major simplification.

Finally, we outlined an approach to universal faulttolerant quantum computing with number-phase codes in Sec. VIII. Given the highly nonlinear nature of the numbertranslation symmetry $\hat{\Sigma}_{N}$ [Eq. (18)], one may wonder whether fault-tolerant error correction is possible. We showed that fault tolerance can indeed be achieved, through concatenation with a Bacon-Shor code and exploiting schemes that were originally developed to deal with highly asymmetric noise $[36,37,106]$. Our scheme illustrates the broader point of how a concatenated scheme can be tailored 
to the strengths and weaknesses of the underlying bosonic code. We showed how bosonic-code error correction can be seamlessly integrated with the qubit-code error correction and furthermore how fault-tolerant gadgets can be constructed using bosonic operations with minimal error spread or amplification. In this way, one can hope to maximize the advantage of using bosonically encoded qubits over bare qubits at the ground level.

It is worth reiterating that no additional syndrome measurements are required to perform error correction at the bosonic level beyond those needed for the qubit subsystem code itself. In other words, error correction at both levels is performed in one step, such that no additional resources are used compared to if bare qubits were used at the ground level. This situation is an interesting contrast to other recent work on concatenation of GKP codes with topological qubit codes $[13,14]$. In these latter works, dedicated ancillae and syndrome-measurement circuits are introduced to perform bosonic-code error correction, thus incurring more overhead.

It is still largely an open problem to quantify the performance of bosonic codes for fault-tolerant error correction and computing and, thus, their potential to give an improvement over bare qubits. An important part of future research on bosonic codes is therefore to quantitatively study different schemes in a fault-tolerant setting. Our scheme based on concatenation with Bacon-Shor codes illustrates some of the key issues a fault-tolerant scheme for bosonic rotation codes must overcome. An interesting direction for future research is to explore this path further with other concatenated codes, in particular, to develop schemes based on concatenation with topological codes and compare bosonic rotation codes to GKP codes at the ground level, following the work in Refs. [13,14]. It is noteworthy that decoders tailored to bosonic codes at the ground level have already shown promise in theoretical work on GKP codes $[12,13,15]$.

\section{ACKNOWLEDGMENTS}

We thank Victor Albert, Rafael Alexander, Stephen Bartlett, Andrew Doherty, Giulia Ferrini, Jonathan Gross, Anirudh Krishna, Nicolas Menicucci, Stefanus Edgar Tanuarta, Barbara Terhal, Matthew Scott Winnel, and Pei Zeng for valuable discussions and feedback during the preparation of this manuscript and Dumpling King for providing the environment where it was conceived. This work was supported by the Australian Research Council (ARC) via the Centre of Excellence in Engineered Quantum Systems (EQUS) Project No. CE170100009, the Centre of Excellence in Quantum Computation and Communication Technology $\left(\mathrm{CQC}^{2} \mathrm{~T}\right)$ Project No. CE170100012, and two Discovery Early Career Research Awards (DECRA) Projects No. DE160100356 and No. DE190100380.

\section{APPENDIX A: DUAL-BASIS PRIMITIVES}

The dual-basis code words $\left| \pm_{N}\right\rangle$ can themselves be described by a set of rotated dual primitives $e^{i \theta \hat{n}}\left|\Theta^{\prime}\right\rangle$ parameterized by $N$ equally spaced angles $\theta=2 m \pi / N$ for $m=0, \ldots, N-1$ :

$$
\begin{aligned}
\left|+_{N, \Theta}\right\rangle & :=\frac{1}{\sqrt{\mathcal{N}_{+}}} \sum_{m=0}^{N-1} e^{i(2 m \pi / N) \hat{n}}\left|\Theta^{\prime}\right\rangle, \\
\left|-_{N, \Theta}\right\rangle & :=\frac{1}{\sqrt{\mathcal{N}_{-}}} \sum_{m=0}^{N-1} e^{i[(2 m+1) \pi / N] \hat{n}}\left|\Theta^{\prime}\right\rangle .
\end{aligned}
$$

The dual primitive $\left|\Theta^{\prime}\right\rangle$ is a weighted superposition of the original primitive $|\Theta\rangle$ with itself rotated by $\pi / N$,

$$
\left|\Theta^{\prime}\right\rangle=\frac{1}{\sqrt{\mathcal{N}_{\Theta^{\prime}}}}\left(c_{+}+c_{-} e^{i(\pi / N) \hat{n}}\right)|\Theta\rangle,
$$

where $c_{ \pm}:=\sqrt{\mathcal{N}_{1}} \pm \sqrt{\mathcal{N}_{0}}$, and the normalization is

$\mathcal{N}_{\Theta^{\prime}}=2\left(\mathcal{N}_{0}+\mathcal{N}_{1}\right)+2\left(\mathcal{N}_{1}-\mathcal{N}_{0}\right)\left\langle\Theta\left|\cos \left(\frac{2 \pi}{N} \hat{n}\right)\right| \Theta\right\rangle$.

Note that, for a fixed primitive $|\Theta\rangle$, the dual primitive $\left|\Theta^{\prime}\right\rangle$ is, in general, different for each $N$.

When the normalization constants satisfy $\mathcal{N}_{0}=\mathcal{N}_{1}$, the computational- and dual-basis primitives coincide. This equality holds for the number-phase codes in Table I in the large average excitation-number limit. An example is cat codes in the limit $\alpha \rightarrow \infty$. In this case, the code states in Eq. (A1) can be expressed simply as

$$
\begin{gathered}
\left|+_{N}\right\rangle \rightarrow \frac{1}{\sqrt{N}} \sum_{m=0}^{N-1} e^{i(2 m \pi / N) \hat{n}}|\Theta\rangle, \\
\left|-{ }_{N}\right\rangle \rightarrow \frac{1}{\sqrt{N}} \sum_{m=0}^{N-1} e^{i[(2 m+1) \pi / N] \hat{n}}|\Theta\rangle,
\end{gathered}
$$

with a set of angles $m(2 \pi / N)$ that are twice the size of those in the computation-basis code words. Moreover, we have that codes of different order are related via

$$
\left|0_{N, \Theta}\right\rangle=\left|+_{2 N, \Theta}\right\rangle \quad\left(\mathcal{N}_{0}=\mathcal{N}_{1}\right) .
$$

\section{APPENDIX B: EXAMPLES OF ROTATION CODES}

The angular and Fock-space structures that characterize rotation codes leave freedom to define different codes through the coefficients $\left\{f_{k N}\right\}$ [Eq. (4)] that arise from a 
choice of primitive $|\Theta\rangle$. Several rotation codes are presented in the literature, such as cat codes [5] and binomial codes [6] and modified versions of these codes that improve their error-correcting capability by changing the relative phases of their Fock-grid coefficients [10]. Their structure, properties, and error resilience have been studied recently in Ref. [44]. We summarize cat and binomial codes here and introduce two other examples. The first is a generalization of cat codes to include squeezing. The second is a new class of code based on the phase states introduced by Pegg and Barnett [69]. For each, we give a primitive $|\Theta\rangle$ and the Fock-grid coefficients $\left\{f_{k N}\right\}$ associated with an order- $N$ code. For binomial codes, the primitive itself depends on $N$, while the other code families use the same primitive for all $N$. Note that in the main text we are primarily interested in the subset of rotation codes that have approximate number-translation symmetry-the numberphase codes introduced in Sec. III. Each of the codes introduced here can serve as a number-phase code in an appropriate limit.

\section{Squeezed cat codes}

Cat codes consist of coherent states superposed in phase space, which can be generalized in a straightforward way to include squeezing. A displaced, squeezed vacuum state is given by

$$
|\alpha, r, \phi\rangle:=\hat{D}(\alpha) \hat{S}(\zeta)|0\rangle,
$$

where $\hat{D}(\alpha)=e^{\alpha a^{\dagger}-\alpha^{*} a}$ is the displacement operator and $\hat{S}(\zeta)=e^{(1 / 2)\left(\zeta a^{\dagger 2}-\zeta^{*} a^{2}\right)}$ is the squeeze operator with squeezing parameter $\zeta=r e^{-2 i \phi}$, where $r$ is the squeezing amplitude and $\phi$ the squeezing angle. The Fock-space representation for a single displaced squeezed vacuum state, $|\alpha, r, \phi\rangle=\sum_{n=0}^{\infty} \tilde{c}_{n}|n\rangle$, is given by the coefficients

$$
\tilde{c}_{n}=\sum_{\ell=0}^{\infty} D_{n, \ell}(\alpha) S_{\ell, 0}(r, \phi),
$$

where the squeezed vacuum coefficients are $S_{\ell, 0}(r, \phi)=0$ for $\ell$ odd and

$$
S_{\ell, 0}(r, \phi)=\sqrt{\operatorname{sech} r}\left(-\frac{1}{2} e^{2 i \phi} \tanh r\right)^{\ell / 2} \frac{\sqrt{\ell !}}{(\ell / 2) !}
$$

for $\ell$ even. In the limit of no squeezing, $S_{\ell, 0}(r \rightarrow 0, \phi)=$ $\delta_{\ell, 0}$. The coefficients for a displaced number state are

$$
D_{n, \ell}(\alpha)=e^{-(1 / 2)|\alpha|^{2}}\left(\frac{\ell !}{n !}\right)^{1 / 2} \alpha^{n-\ell} \mathcal{L}_{\ell}^{n-\ell}\left(|\alpha|^{2}\right)
$$

with associated Laguerre polynomials $\mathcal{L}_{\ell}^{k}(x)$.
The $\left|0_{N}\right\rangle$ and $\left|1_{N}\right\rangle$ code words are constructed using a displaced squeezed state as the primitive $\left|\Theta_{\text {scat }}\right\rangle=$ $|\alpha, r, \phi=0\rangle$ with $\alpha$ real and positive. From Eq. (3a), the computational basis states are given by [118]

$$
\begin{gathered}
\left|0_{N, \text { scat }}\right\rangle:=\frac{1}{\sqrt{\mathcal{N}_{0}}} \sum_{m=0}^{2 N-1}\left|\alpha e^{i m(\pi / N)}, r, m \frac{\pi}{N}\right\rangle, \\
\left|1_{N, \text { scat }}\right\rangle:=\frac{1}{\sqrt{\mathcal{N}_{1}}} \sum_{m=0}^{2 N-1}(-1)^{m}\left|\alpha e^{i m(\pi / N)}, r, m \frac{\pi}{N}\right\rangle .
\end{gathered}
$$

The Fock-grid coefficients can be found readily from the $\tilde{c}_{n}$ in Eq. (B2); see Sec. II A for details.

Two code subclasses can be identified by specific parameters: Cat codes emerge in the limit that squeezing vanishes, $r=0$, and squeezed vacuum codes arise when the displacement vanishes, $\alpha=0$ [120]. For the smallest cat code $(\alpha \rightarrow 0, r=0)$, the trivial encoding in Eq. (12) is realized. The Fock-grid coefficients for cat codes are

$$
f_{k N}=\sqrt{\frac{2}{\mathcal{N}_{i}^{*}}} e^{-(1 / 2)|\alpha|^{2}} \frac{\alpha^{k N}}{\sqrt{(k N) !}},
$$

where $\mathcal{N}_{i}^{*}$ indicates the Fock-space normalization factor $\mathcal{N}_{0}^{*}\left(\mathcal{N}_{1}^{*}\right)$ (see Sec. II A) for $k$ even (odd).

Note that the cat codes we consider in this work are distinct from the two-mode cat codes in Ref. [48] and the single-mode cat codes in Refs. [4,121], where the code words are manifestly nonorthogonal while still exhibiting discrete rotational symmetry.

\section{Binomial codes}

Binomial codes are introduced in Ref. [6] as a class of codes that can exactly correct loss, gain, and dephasing errors up to a certain order. The code words are most straightforwardly defined in the conjugate basis [122]:

$$
\begin{gathered}
\left|+_{N, \text { bin }}\right\rangle:=\sum_{k=0}^{K} \sqrt{\frac{1}{2^{K}}\left(\begin{array}{c}
K \\
k
\end{array}\right)}|k N\rangle, \\
\left|-_{N, \text { bin }}\right\rangle:=\sum_{k=0}^{K}(-1)^{k} \sqrt{\frac{1}{2^{K}}\left(\begin{array}{l}
K \\
k
\end{array}\right)}|k N\rangle .
\end{gathered}
$$

The Fock-grid coefficients $\left\{f_{k N}\right\}$ can be read off from the computational basis states:

$$
\left|0_{N, \text { bin }}\right\rangle=\sum_{k=0}^{\lfloor K / 2\rfloor} \sqrt{\frac{1}{2^{K-1}}\left(\begin{array}{c}
K \\
2 k
\end{array}\right)}|2 k N\rangle,
$$




$$
\left|1_{N, \text { bin }}\right\rangle=\sum_{k=0}^{\lfloor K / 2\rfloor-1} \sqrt{\frac{1}{2^{K-1}}\left(\begin{array}{c}
K \\
2 k+1
\end{array}\right)}|(2 k+1) N\rangle,
$$

where $\lfloor x\rfloor$ is the floor function that gives the largest integer less than or equal to the real number $x$. There are many binomial codes with $N$-fold rotation symmetry, one for each value of $K=1,2, \ldots$, which sets the truncation level in Fock space. The mean excitation number is $\bar{n}_{\text {bin }}=\frac{1}{2} N K$ [44], and the mean modular phase can be simplified to

$$
\left\langle e^{i \theta N}\right\rangle=\frac{1}{2^{K}} \sum_{k=0}^{K-1} \sqrt{\frac{K-k}{k+1}}\left(\begin{array}{l}
K \\
k
\end{array}\right) .
$$

Binomial codes are defined explicitly at the code word level, leaving freedom to describe associated primitives. However, each binomial code, specified by its rotational order $N$ and truncation parameter $K$, has a different set of primitives. An example primitive can be defined as

$$
\left|\Theta_{\text {bin }}^{N, K}\right\rangle=\frac{1}{\sqrt{\mathcal{N}_{\text {bin }}}} \sum_{n=0}^{K N} \sqrt{\frac{1}{2^{K-1}}\left(\begin{array}{c}
K \\
\lfloor n / N\rfloor
\end{array}\right)},
$$

with $\mathcal{N}_{\text {bin }}$ a normalization constant. The smallest binomial codes $(K=1)$ yield the $0 \mathrm{~N}$ code $\left|+_{N}\right\rangle=(1 / \sqrt{2})(|0\rangle+|N\rangle)$, which is the trivial encoding [Eq. (12)] for $N=1$.

\section{Pegg-Barnett codes}

A Pegg-Barnett phase state is given by [69]

$$
|\phi, s\rangle:=\frac{1}{\sqrt{s}} \sum_{n=0}^{s-1} e^{i n \phi}|n\rangle,
$$

where the parameter $s$ sets the truncation level. The set of states $\left\{\left|\phi_{m}=2 \pi m / s, s\right\rangle\right\}$ with $m=0, \ldots, s-1$ forms an orthonormal basis for the $s$-dimensional truncated Fock space.

We use a specific phase state as a primitive, $\left|\Theta_{\mathrm{pb}}\right\rangle=|\phi=0, s\rangle$, to define Pegg-Barnett codes. To ensure adequate Fock space for the code words, the truncation $s-1$ is required to be at least $N$. Given that $e^{i \theta \hat{n}}|\phi, s\rangle=|\phi+\theta, s\rangle$, the code words can be expressed simply as

$$
\begin{array}{r}
\left|0_{N, \mathrm{pb}}\right\rangle:=\frac{1}{\sqrt{\mathcal{N}_{0}}} \sum_{m=0}^{2 N-1}\left|\phi=m \frac{\pi}{N}, s\right\rangle, \\
\left|1_{N, \mathrm{pb}}\right\rangle:=\frac{1}{\sqrt{\mathcal{N}_{1}}} \sum_{m=0}^{2 N-1}(-1)^{m}\left|\phi=m \frac{\pi}{N}, s\right\rangle .
\end{array}
$$

Note that if the truncation of the Pegg-Barnett phase state used as a primitive is commensurate with the order of the code, $s=p \times 2 N$ for $p=1,2,3, \ldots$, then the rotated primitives are automatically orthogonal, $\langle\phi=m(\pi / N)$, $s\left|\phi=m^{\prime}(\pi / N), s\right\rangle=\delta_{m, m^{\prime}}$. In this case, the conjugatebasis code words and the dual-basis code words are simple superpositions of the primitive; cf. Eq. (A4). For $s=p \times 2 N$, we also have that the Pegg-Barnett codes can be recognized as the shift-resistant qudit codes from Ref. [3], with $d=s, n=2, r_{1}=N$, and $r_{2}=p$, using the notation from Ref. [3].

The Fock-grid coefficients, in general, are $f_{k N}=$ $\sqrt{2 /\lceil s / N\rceil}$, where $\lceil x\rceil$ is the ceiling function that gives the least integer greater than or equal to the real number $x$. The mean excitation number is $\bar{n}_{\mathrm{pb}}=(N / 2)(\lceil s / N\rceil-1)$, and the mean modular phase is

$$
\left\langle e^{i N \theta}\right\rangle=1-\frac{1}{\lceil s / N\rceil} .
$$

The Pegg-Barnett code $[N ; s=N+1]$ is the ON code, and the smallest Pegg-Barnett code $[N=1 ; s=2]$ is the trivial encoding [Eq. (12)].

\section{APPENDIX C: ROTATION SYMMETRY FOR GOTTESMAN-KITAEV-PRESKILL CODES}

Logical Pauli gates for GKP codes are realized by discrete translations in phase space. As a consequence of translation symmetry, all GKP codes also exhibit $N=2$ discrete rotation symmetry, as we show below. However, GKP codes are not rotation codes as we define them in Sec. II, because the operator $\hat{Z}_{2}$ does not act as a Pauli operator. Depending on the underlying lattice defining the GKP code words, $\hat{Z}_{N}$ may nevertheless act as a logical Clifford operator for some values of $N$. Two notable GKP codes where such is the case are the square- and hexagonallattice GKP codes, which means that these codes exhibit some properties of rotation codes. We briefly elucidate this point.

The code space of a single-mode ideal GKP qubit is defined to be the +1 eigenspace of the stabilizers

$$
\hat{S}_{\text {gkp }}^{X}:=\hat{D}(2 \alpha), \quad \hat{S}_{\text {gkp }}^{Z}:=\hat{D}(2 \beta),
$$

where $\hat{D}(\zeta)=e^{\zeta \hat{a}^{\dagger}-\zeta^{*} \hat{a}}$ is the displacement operator [123] and $\alpha$ and $\beta$ are complex numbers satisfying

$$
\alpha^{*} \beta-\alpha \beta^{*}=i \pi
$$

The stabilizer group consists of all powers $\left(\hat{S}_{\text {gkp }}^{X}\right)^{n_{1}}\left(\hat{S}_{\text {gkp }}^{Z}\right)^{n_{2}}$ for $n_{1}, n_{2} \in \mathbb{Z}$. The shift operators that act as logical Paulis for the GKP qubit are

$$
\begin{aligned}
& \hat{X}_{\mathrm{gkp}}:=\hat{D}(\alpha), \\
& \hat{Z}_{\mathrm{gkp}}:=\hat{D}(\beta),
\end{aligned}
$$




$$
\hat{Y}_{\mathrm{gkp}}:=i \hat{Z}_{\mathrm{gkp}} \hat{X}_{\mathrm{gkp}}=\hat{D}(\alpha+\beta),
$$

where the form of $\hat{Y}_{\text {gkp }}$ follows from the general identity $\hat{D}(\alpha) \hat{D}(\beta)=e^{(1 / 2)\left(\alpha \beta^{*}-\alpha^{*} \beta\right)} \hat{D}(\alpha+\beta)$. The constraint Eq. (C2) ensures that the stabilizers in Eq. (C1) commute and that $\hat{X}_{\text {gkp }}$ and $\hat{Z}_{\text {gkp }}$ commute with the stabilizers and anticommute with each other.

From the identity $e^{-i \theta \hat{n}} \hat{D}(\zeta) e^{i \theta \hat{n}}=\hat{D}\left(e^{-i \theta} \zeta\right)$, we see that the discrete rotation operator $\hat{R}_{N}$ for $N=2$ has the following action on the Pauli shift operators:

$$
\begin{aligned}
& \hat{R}_{2}^{\dagger} \hat{X}_{\text {gkp }} \hat{R}_{2}=\hat{D}(-\alpha)=\hat{X}_{\text {gkp }}^{\dagger}, \\
& \hat{R}_{2}^{\dagger} \hat{Z}_{\text {gkp }} \hat{R}_{2}=\hat{D}(-\beta)=\hat{Z}_{\text {gkp }}^{\dagger} .
\end{aligned}
$$

Since $\hat{X}_{\text {gkp }}^{\dagger}=\hat{X}_{\text {gkp }}\left(\hat{S}_{\text {gkp }}^{X}\right)^{-1}$ and $\hat{Z}_{\text {gkp }}^{\dagger}=\hat{Z}_{\text {gkp }}\left(\hat{S}_{\text {gkp }}^{Z}\right)^{-1}$, they are equivalent up to stabilizers and have the same action on the code space. Thus, $\hat{R}_{2}$ acts as the identity operator on the code space of an ideal GKP code. In other words, all GKP codes have twofold discrete rotation symmetry.

The computational-basis code words can be written as a superposition of coherent states:

$$
\begin{aligned}
\left|j_{\mathrm{gkp}}\right\rangle & =\sum_{n_{1}, n_{2} \in \mathbb{Z}} \hat{D}\left[\left(2 n_{1}+j\right) \alpha\right] \hat{D}\left(n_{2} \beta\right)|0\rangle \\
& =\sum_{n_{1}, n_{2} \in \mathbb{Z}} e^{-i \pi\left(n_{1} n_{2}+j n_{2} / 2\right)}\left|\left(2 n_{1}+j\right) \alpha+n_{2} \beta\right\rangle,
\end{aligned}
$$

with $j=0,1$. It is natural to define state lattices in the complex plane for the computational-basis code words,

$$
\mathcal{L}_{j}=\left\{\left(2 n_{1}+j\right) \alpha+n_{2} \beta \mid n_{1}, n_{2} \in \mathbb{Z}\right\},
$$

as well as the code space lattice, $\mathcal{L}=\mathcal{L}_{0} \cup \mathcal{L}_{1}$, consisting of all points $n_{1} \alpha+n_{2} \beta$. As described in Ref. [44], the code word lattices are basis dependent, while the code space lattice $\mathcal{L}$ is not. Note that Eq. (C2) means that the unit cell, or fundamental parallelogram, of $\mathcal{L}$ has area $\pi / 2$ [124].

We consider two canonical examples of ideal GKP codes defined on square ( $g k p s)$ and hexagonal ( $g k p h)$ lattices. The codes can be defined in terms of their respective latticebasis translations $\alpha$ and $\beta$ :

$$
\begin{array}{r}
\text { gkps: } \alpha=\sqrt{\frac{\pi}{2}}, \quad \beta=i \sqrt{\frac{\pi}{2}}, \\
\text { gkph: } \alpha=\sqrt{\frac{\pi}{\sqrt{3}}}, \quad \beta=e^{i(2 \pi / 3)} \sqrt{\frac{\pi}{\sqrt{3}}} .
\end{array}
$$

Code space lattices for gkps and gkph are shown in Fig. 13. (a)
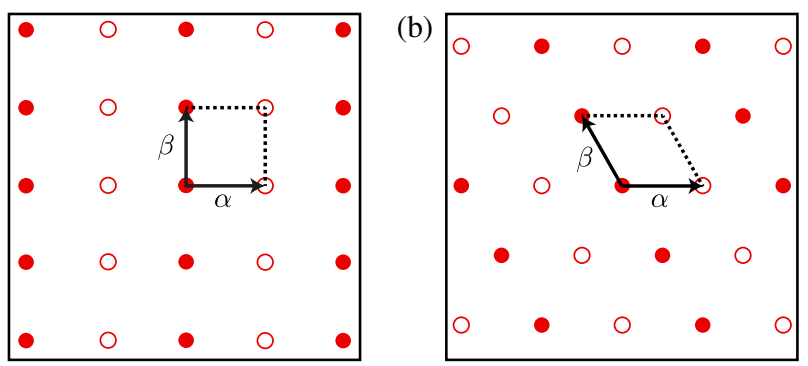

FIG. 13. Code lattices $\mathcal{L}_{0}$ (filled circles) and $\mathcal{L}_{1}$ (open circles) in the complex plane for (a) square-lattice GKP and (b) hexagonal-lattice GKP. The area of the lattice parallelogram is $\pi / 2$. The code space lattice $\mathcal{L}$ is also apparent in the Wigner function $W_{\text {gkp }}(\alpha)$ of the completely mixed state $\frac{1}{2} \hat{\Pi}_{\text {gkp }}$. Using quadrature axes $Q$ and $I$, as defined in Fig. $1, W_{\mathrm{gkp}}(\alpha)$ is identical to the above plots with positive $\delta$ functions at each point in $\mathcal{L}$. For the Wigner functions, arrows indicate the direction of displacement for $\hat{X}_{\text {gkp }}$ and $\hat{Z}_{\text {gkp }}$.

Depending on the lattice, specific discrete rotations can act as nontrivial logical Clifford gates. In particular, for square-lattice GKP, $N=4$ rotations transform the Paulis:

$$
\begin{aligned}
& \hat{R}_{4}^{\dagger} \hat{X}_{\text {gkps }} \hat{R}_{4}=\hat{Z}_{\text {gkps }}^{\dagger}, \\
& \hat{R}_{4}^{\dagger} \hat{Z}_{\text {gkps }} \hat{R}_{4}=\hat{X}_{\text {gkps }} .
\end{aligned}
$$

Thus, $\hat{R}_{4}=\hat{Z}_{2}=e^{i(\pi / 2) \hat{n}}$ (the Fourier transform operator) acts as a logical Hadamard $\bar{H}$ for gkps. It follows that the eigenstates of $\bar{H}$ for this code can be written in the form Eq. (4), a fact that is recognized in Ref. [3]. It is also suggested there that the measurement of number mod 4 can be used to prepare Hadamard eigenstates, which are equivalent to the magic state $\left|T_{\text {gkps }}\right\rangle$ up to Cliffords. Since mapping logical $\bar{H}$ to $\bar{Z}$ is a non-Clifford operation, this mapping is not a trivial change of basis for a GKP code, and we therefore do not classify the square-lattice GKP code as a rotation code.

For hexagonal-lattice GKP, $N=6$ rotations perform logical $\pi / 3$ rotations around the $X Y Z$ axis:

$$
\begin{aligned}
& \hat{R}_{6}^{\dagger} \hat{X}_{\text {gkph }} \hat{R}_{6}=\hat{Z}_{\text {gkph }}^{\dagger}, \\
& \hat{R}_{6}^{\dagger} \hat{Z}_{\text {gkph }} \hat{R}_{6}=\hat{Y}_{\text {gkph }},
\end{aligned}
$$

giving a cyclic permutation of the logical Pauli operators. Thus, $\hat{R}_{6}=\hat{Z}_{3}$ acts as the logical Clifford gate $\bar{H} \bar{S}^{\dagger}$ [125]. An $N=3$ rotation, given by $\hat{R}_{3}=\hat{R}_{6}^{2}$, acts as the logical gate $\left(\bar{H} \bar{S}^{\dagger}\right)^{2}=e^{-i(\pi / 4)} \bar{S} \bar{H}$, which performs a cyclic permutation of the Paulis in the opposite order.

The fact that the square- and hexagonal-lattice GKP codes have nontrivial Cliffords implementable as discrete rotations also implies that the CROT gate can be used between a bosonic rotation code and these two GKP codes. 
In particular, $\mathrm{CROT}_{N 4}\left(\mathrm{CROT}_{N 6}\right)$ implements a controlled$\bar{H}$ (controlled- $\bar{H} \bar{S}^{\dagger}$ ) between an order- $N$ rotation code and a square (hexagonal) GKP code, with the rotation code as the control and the GKP code as the target.

In contrast to square- and hexagonal-, the rectangularlattice GKP code does not benefit from additional Clifford gates performed via discrete rotations. Last, we note that the crystallographic restriction theorem implies that no lattice can have higher than sixfold rotation symmetry, and $\hat{R}_{6}$ is the highest degree rotation operator that can preserve the code space of a GKP code.

\section{APPENDIX D: PRETTY GOOD MEASUREMENTS}

As we allude to in Sec. II C2, the canonical phase measurement might not be the optimal measurement to distinguish the code words $\left| \pm_{N}\right\rangle$. Even in the absence of noise, two orthogonal code words cannot, in general, be perfectly distinguished by a canonical phase measurement due to embedded phase uncertainty $\Delta_{N}(\theta)$. Moreover, in a realistic scenario where the code words may be damaged, defining a phase-estimation problem with respect to the ideal code words is suboptimal. We do not attempt to find an optimal scheme, in general, as this must likely be done on a case-by-case basis for different codes and noise models. Instead, we introduce a measurement that performs well for all the codes and noise models we study in this paper. This measurement is the "pretty good measurement" introduced in Refs. [51-53]. The "pretty good measurement" perfectly distinguishes orthogonal states and is designed to also distinguish fairly well states with small nonzero overlaps. The measurement is defined through the POVM elements

$$
\hat{M}_{i}^{\text {pretty good }}:=\hat{\sigma}^{-1 / 2} \mathcal{N}(|i\rangle\langle i|) \hat{\sigma}^{-1 / 2},
$$

where $|i\rangle$ runs over the states to be distinguished and $\mathcal{N}$ represents an arbitrary noise channel. The operator $\hat{\sigma}=$ $\mathcal{N}(\hat{P})$ with $\hat{P}=\sum_{i}|i\rangle\langle i|$ represents the projector onto the subspace in question sent through the noise channel. The POVM elements satisfy $\sum_{i} \hat{M}_{i}=\hat{P}_{\sigma}$, where $\hat{P}_{\sigma}$ is a projector onto the support of $\hat{\sigma}$. To have a complete measurement, one can add a POVM element $\hat{I}-\hat{P}_{\sigma}$ projecting onto the complement of $\hat{P}_{\sigma}$. Note that, in contrast to the canonical phase measurement, the pretty

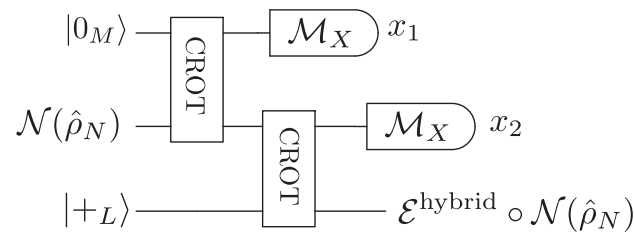

FIG. 14. A hybrid Steane-Knill error-correction scheme for rotation codes. good measurement explicitly exploits knowledge about the noise $\mathcal{N}$.

\section{APPENDIX E: HYBRID STEANE-KNILL ERROR CORRECTION}

Here, we introduce an error-correction circuit, depicted in Fig. 14, as an alternative to the circuit in Fig. 7. We call this error-correction method "hybrid" (hybrid-EC), because the first CROT gate and the measurement of the top ancilla rail can be recognized as identical to one of the two steps of Steane-EC, while the second CROT and the measurement on the data rail is a one-bit teleportation, identical to one of the steps in Knill-EC. Essentially, this circuit uses Steane-EC for one of the syndromes (number-shift errors) and Knill$\mathrm{EC}$ for the other (dephasing errors).

This error-correction scheme is based on the circuit identity

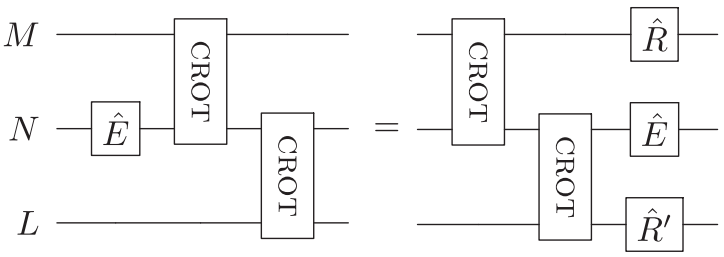

where $\hat{E}=\hat{E}_{k}(\theta)$ is again an arbitrary initial error and the two rotation errors $\hat{R}=\hat{E}_{0}(\pi k / N M)$ and $\hat{R}^{\prime}=\hat{E}_{0}(\pi k / N L)$ are both proportional to $k$. A phase measurement on the top rail thus estimates $k$, and a recovery operation can be applied to the bottom rail to undo the leftover error $\hat{R}^{\prime}$.

The first step of the circuit in Fig. 7(b), involving only the top ancilla,

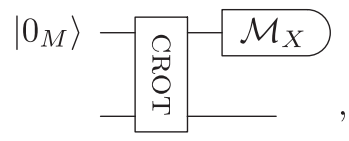

performs a nondestructive measurement of the excitation number $\bmod N$ on the data rail, as explained in Sec. V. The second step of the circuit,

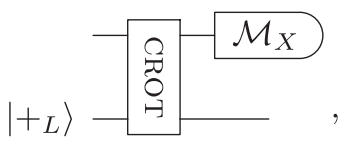

teleports the data to a fresh new ancilla, thus restoring the code space up to a known rotation error as per Eq. (E1).

More explicitly, we consider an encoded state $\hat{\rho}_{N}$ in an order- $N$ rotation code sent through a noise channel $\mathcal{N} \cdot=\sum_{n} \hat{A}_{n} \cdot \hat{A}_{n}^{\dagger}$, followed by the error-correction circuit in Fig. 14. In this case, it is convenient to expand the noise Kraus operators as $\hat{A}_{n}=\sum_{k} \int d \theta c_{n k}(\theta) \hat{E}_{k}(\theta)=\sum_{k} \hat{E}_{n k}$, where we define $\hat{E}_{n k}=\int d \theta c_{n k}(\theta) \hat{E}_{k}(\theta)$. The state after the error-correction circuit can be written 


$$
\begin{aligned}
\mathcal{E}^{\text {hybrid }} \circ \mathcal{N}\left(\hat{\rho}_{N}\right)= & \frac{1}{2} \sum_{a, b= \pm} \sum_{\vec{x}} \sum_{n, k, k^{\prime}} \operatorname{tr}\left[\hat{M}_{x_{1}} \hat{\sigma}_{a b}^{n k k^{\prime}}\right] \operatorname{tr}\left[\hat{M}_{x_{2}} \hat{\tau}^{k k^{\prime}}\right] \\
& \times \hat{E}_{0}\left(\frac{\pi k}{N L}\right) \bar{C}_{a} \hat{\rho}_{L} \bar{C}_{b}^{\dagger} \hat{E}_{0}^{\dagger}\left(\frac{\pi k^{\prime}}{N L}\right),
\end{aligned}
$$

where

$$
\begin{gathered}
\hat{\sigma}_{a b}^{n k k^{\prime}}:=\hat{E}_{n k}\left|a_{N}\right\rangle\left\langle b_{N}\right| \hat{E}_{n k^{\prime}}^{\dagger}, \\
\hat{\tau}^{k k^{\prime}}:=\hat{E}_{0}\left(\frac{\pi k}{N M}\right)\left|0_{M}\right\rangle\left\langle 0_{M}\right| \hat{E}_{0}^{\dagger}\left(\frac{\pi k^{\prime}}{N M}\right)
\end{gathered}
$$

represent damaged code words for the data and ancilla, respectively, and $\bar{C}_{+}:=\bar{H}, \bar{C}_{-}:=\bar{X} \bar{H}$ are logical Cliffords. We again use a notation where $\hat{\rho}_{L}$ on the right-hand side represents the same logical state as $\hat{\rho}_{N}$ on the left-hand side but where the latter is encoded in the order- $L$ number-phase code of the bottom ancilla in Fig. 14.

The most likely rotation error on the output, $\hat{E}_{0}\left(\pi k^{*} / N L\right)$, as well as the most likely logical Clifford error $\bar{C}_{a^{*}}$ can be deduced using, e.g., a maximum likelihood decoder. The rotation error is straightforward to correct or, alternatively, can be tracked by updating later phase measurements. The error $\bar{C}_{a^{*}}$ is not a Pauli error; however, by performing the error-correction circuit twice, the most likely error will be a Pauli from the set $\bar{P}_{i} \in\{\bar{I}, \bar{Z}, \bar{X}, \bar{X} \bar{Z}\}$ which can be tracked in a Pauli frame.

We have verified in numerical simulations that the hybrid-EC scheme performs identically to the Knill-EC scheme presented in Sec. VII A; i.e., the results in Sec. VII B are identical for both schemes. In fact, there is arguably a slight advantage of the hybrid-EC scheme in the case of noiseless ancillae, because we can use an unencoded state $|\alpha\rangle$ for the upper ancilla rail in Fig. 14. This state give slightly better phase resolution compared to an $M=1$ cat code, as used in Sec. VII B, at the same value of $\alpha$.

\section{Relation to recent experiments}

The hybrid-EC scheme generalizes the experimental protocol used in the two experiments in Refs. [29,30], where error correction for $N=2$ cat and binomial codes is implemented, respectively. The scheme employs the error-syndrome circuit Eq. (E2) with the ancilla replaced by an unencoded qubit in the state $|+\rangle=(|0\rangle+|1\rangle) / \sqrt{2}$, and the CROT gate in their case is CROT $=\mathrm{CROT}_{N 1 / 2}=$ $e^{i(2 \pi / N) \hat{n} \otimes \hat{n}}$. An error $E_{k}(\theta)$ on the data qubit is thus imprinted as a phase on the ancilla, i.e., $|+\rangle \rightarrow|0\rangle+$ $e^{i(2 \pi k / N)}|1\rangle$, and the syndrome measurement attempts to estimate this phase. Note that, with $N=2$, an initial error prior to the gate with $|k| \bmod 2=1$ on the data qubit flips the ancilla to $|-\rangle$, while an error with $|k| \bmod 2=0$ leaves the ancilla in the state $|+\rangle$. This syndrome does not detect dephasing errors, nor does it restore the state back to the Fock grid $|k N\rangle$. For cat codes, loss events can be tracked in software so that recoveries for this particular error are not necessary (this does not include the no-jump part of the bosonic loss channel, however) [29]. For binomial codes, optimal control is employed in Ref. [30] to find a unitary that approximately restores the code space. In the hybrid-EC scheme we propose, both the detection of dephasing errors and the restoration of the code space are performed by the second step of the errorcorrection circuit [Eq. (E3)]. The difficulty of restoring the code space has thus been replaced by the ability to prepare copies of encoded $\left|+_{N}\right\rangle$ states.

\section{APPENDIX F: LOSS AND DEPHASING CHANNEL}

We seek a convenient representation of the channel that describes simultaneous loss and dephasing:

$$
\mathcal{N}=e^{t\left(\mathcal{L}_{\kappa}+\mathcal{L}_{\kappa_{\phi}}\right)},
$$

where $\mathcal{L}_{\kappa}:=\kappa \mathcal{D}[\hat{a}]$ and $\mathcal{L}_{\kappa_{\phi}}:=\kappa_{\phi} \mathcal{D}[\hat{n}]$; cf. Eq. (49). First, we note that the pure dephasing channel can be written

$$
e^{t \mathcal{L}_{\kappa_{\phi}} \hat{\rho}}=\int_{-\infty}^{\infty} d \theta p_{\kappa_{\phi} t}(\theta) e^{i \theta \hat{n}} \hat{\rho} e^{-i \theta \hat{n}}
$$

where $p_{\kappa_{\phi} t}=\left(2 \pi \kappa_{\phi} t\right)^{-1 / 2} e^{-\theta^{2} /\left(2 \kappa_{\phi} t\right)}$ is a Gaussian with zero mean and variance $\kappa_{\phi} t$. This result can be shown by first considering the small $\kappa_{\phi} t$ limit:

$$
\begin{aligned}
e^{\mathcal{L}_{\kappa_{\phi}} t} \hat{\rho} & \simeq \int_{-\infty}^{\infty} d \theta p_{\kappa_{\phi} t}(\theta)(1+i \theta \hat{n}) \hat{\rho}(1-i \theta \hat{n}) \\
& \simeq \int_{-\infty}^{\infty} d \theta p(\theta)\left[e^{i \theta \hat{n}} \hat{\rho}+\hat{\rho} e^{-i \theta \hat{n}}+\theta^{2} \hat{n} \hat{\rho} \hat{n}-\hat{\rho}\right] \\
& =e^{-(1 / 2) \kappa_{\phi} t^{2}} \hat{\rho}+\hat{\rho} e^{-(1 / 2) \kappa_{\phi} t \hat{n}^{2}}+\kappa_{\phi} t \hat{n} \hat{\rho} \hat{n}-\hat{\rho} \\
& \simeq \hat{\rho}-\frac{1}{2} \kappa_{\phi} t \hat{n}^{2} \hat{\rho}-\frac{1}{2} \hat{\rho} \kappa_{\phi} t \hat{n}^{2}+\kappa_{\phi} t \hat{n} \rho \hat{n},
\end{aligned}
$$

and thus

$$
\dot{\hat{\rho}}=\lim _{t \rightarrow 0} \frac{e^{t \mathcal{L}_{\kappa_{\phi}}} \hat{\rho}-\hat{\rho}}{t}=\mathcal{L}_{\kappa_{\phi}} \hat{\rho},
$$

as required.

Returning to Eq. (F1), we can write the solution $\hat{\rho}(t)=$ $\mathcal{N}\left(\hat{\rho}_{0}\right)$ in terms of a generalized Dyson expansion [126,127]:

$$
\begin{aligned}
\hat{\rho}(t)= & \sum_{k=0}^{\infty} \int_{0}^{t} d t_{k} \int_{0}^{t_{k}} d t_{k-1} \ldots \int_{0}^{t_{2}} d t_{1} \\
& \times e^{\left(t-t_{k}\right) \mathcal{S}} \mathcal{J} \ldots \mathcal{J} e^{\left(t_{2}-t_{1}\right) \mathcal{S}} \mathcal{J} e^{t_{1} \mathcal{S}} \hat{\rho}_{0},
\end{aligned}
$$

where $\mathcal{J} \hat{\rho}=\kappa \hat{a} \hat{\rho} \hat{a}^{\dagger}$ and $\mathcal{S}=\mathcal{L}_{\kappa_{\phi}}+\mathcal{L}_{\kappa}-\mathcal{J}$. Next, note that the no-jump part of the evolution factorizes as

$$
e^{t \mathcal{S}}=e^{t\left(\mathcal{L}_{\kappa_{\phi}}+\mathcal{L}_{\kappa}-\mathcal{J}\right)}=e^{t \mathcal{L}_{\kappa_{\phi}}} e^{t\left(\mathcal{L}_{\kappa}-\mathcal{J}\right)},
$$


where $e^{t \mathcal{L}_{\kappa_{\phi}}}$ is given by Eq. (F2) and

$$
e^{t\left(\mathcal{L}_{\kappa}-\mathcal{J}\right)} \hat{\rho}=e^{-\kappa \hat{n} t / 2} \hat{\rho} e^{-\kappa \hat{n} t / 2} .
$$

Using this result, it is straightforward to pull the jump superoperators $\mathcal{J}$ through all the no-jump superoperators in Eq. (F5):

$$
\begin{gathered}
\mathcal{J} e^{t \mathcal{L}_{\kappa_{\phi}}}=e^{t \mathcal{L}_{\kappa_{\phi}}} \mathcal{J}, \\
\mathcal{J} e^{t\left(\mathcal{L}_{\kappa}-\mathcal{J}\right)}=e^{-\kappa t} e^{t\left(\mathcal{L}_{\kappa}-\mathcal{J}\right)} \mathcal{J} .
\end{gathered}
$$

After pushing all the $\mathcal{J}$ 's to the right, rewriting the $k$ timeordered integrals as $k$ integrals over $[0, t]$, and performing the integrals, one readily finds

$$
\hat{\rho}(t)=e^{t \mathcal{L}_{\kappa_{\phi}}} \sum_{k=0}^{\infty} \hat{A}_{k} \hat{\rho}_{0} \hat{A}_{k}^{\dagger},
$$

where $\hat{A}_{k}$ is given by

$$
\hat{A}_{k}:=\frac{\left(1-e^{-\kappa t}\right)^{k / 2}}{\sqrt{k !}} e^{-\kappa \hat{n} t / 2} \hat{a}^{k} .
$$

One can, of course, also find a Kraus-operator representation of $e^{t \mathcal{L}_{\kappa_{\phi}}}$ using exactly the same method:

$$
e^{t \mathcal{L}_{\kappa_{\phi}}} \hat{\rho}=\sum_{\ell=0}^{\infty} \hat{B}_{\ell} \hat{\rho} \hat{B}_{\ell}^{\dagger}
$$

with

$$
\hat{B}_{\ell}:=\frac{\left(\kappa_{\phi} t\right)^{\ell / 2}}{\sqrt{\ell !}} e^{-(1 / 2) \kappa_{\phi} \hat{n}^{2} t} \hat{n}^{\ell}
$$

We, however, do not need such a Kraus-operator representation of the dephasing channel for our purposes in Sec. VII, since the dephasing channel commutes with all the CROT gates. We find it more convenient to solve the master equation

$$
\dot{\mathcal{N}}_{\kappa_{\phi}}=\mathcal{L}_{\kappa_{\phi}} \mathcal{N}_{\kappa_{\phi}}
$$

numerically, with $\mathcal{N}_{\kappa_{\phi}}(0)=\mathcal{I}$ the identity superoperator as the initial condition, to find the pure dephasing channel $\mathcal{N}_{\kappa_{\phi}}(t)=e^{t \mathcal{L}_{\kappa_{\phi}}}$

[1] I. L. Chuang, D. W. Leung, and Y. Yamamoto, Bosonic Quantum Codes for Amplitude Damping, Phys. Rev. A 56, 1114 (1997).

[2] P. T. Cochrane, G. J. Milburn, and W. J. Munro, Macroscopically Distinct Quantum-Superposition States as a
Bosonic Code for Amplitude Damping, Phys. Rev. A 59, 2631 (1999).

[3] D. Gottesman, A. Kitaev, and J. Preskill, Encoding a Qubit in an Oscillator, Phys. Rev. A 64, 012310 (2001).

[4] A. P. Lund, T. C. Ralph, and H. L. Haselgrove, FaultTolerant Linear Optical Quantum Computing with SmallAmplitude Coherent States, Phys. Rev. Lett. 100, 030503 (2008).

[5] M. Mirrahimi, Z. Leghtas, V. V. Albert, S. Touzard, R. J. Schoelkopf, L. Jiang, and M. H. Devoret, Dynamically Protected Cat-Qubits: A New Paradigm for Universal Quantum Computation, New J. Phys. 16, 045014 (2014).

[6] M. H. Michael, M. Silveri, R. T. Brierley, V. V. Albert, J. Salmilehto, L. Jiang, and S. M. Girvin, New Class of Quantum Error-Correcting Codes for a Bosonic Mode, Phys. Rev. X 6, 031006 (2016).

[7] S. Puri, C. K. Andersen, A. L. Grimsmo, and A. Blais, Quantum Annealing with All-to-All Connected Nonlinear Oscillators, Nat. Commun. 8, 15785 (2017).

[8] S. Puri, A. Grimm, P. Campagne-Ibarcq, A. Eickbusch, K. Noh, G. Roberts, L. Jiang, M. Mirrahimi, M. H. Devoret, and S.M. Girvin, Stabilized Cat in Driven Nonlinear Cavity: A Fault-Tolerant Error Syndrome Detector, Phys. Rev. X 9, 041009 (2019).

[9] K. Noh, V. V. Albert, and L. Jiang, Quantum Capacity Bounds of Gaussian Thermal Loss Channels and Achievable Rates with Gottesman-Kitaev-Preskill Codes, IEEE Trans. Inf. Theory 65, 2563 (2019).

[10] L. Li, D. J. Young, V. V. Albert, K. Noh, C.-L. Zou, and L. Jiang, Designing Good Bosonic Quantum Codes via Creating Destructive Interference, arXiv:1901.05358.

[11] N. C. Menicucci, Fault-Tolerant Measurement-Based Quantum Computing with Continuous-Variable Cluster States, Phys. Rev. Lett. 112, 120504 (2014).

[12] K. Fukui, A. Tomita, and A. Okamoto, Analog Quantum Error Correction with Encoding a Qubit into an Oscillator, Phys. Rev. Lett. 119, 180507 (2017).

[13] C. Vuillot, H. Asasi, Y. Wang, L. P. Pryadko, and B. M. Terhal, Quantum Error Correction with the Toric Gottesman-Kitaev-Preskill Code, Phys. Rev. A 99, 032344 (2019).

[14] K. Noh and C. Chamberland, Fault-Tolerant Bosonic Quantum Error Correction with the Surface-GKP Code, arXiv:1908.03579 [Phys. Rev. A (to be published)].

[15] K. Fukui, A. Tomita, and A. Okamoto, Tracking Quantum Error Correction, Phys. Rev. A 98, 022326 (2018).

[16] H. J. Kimble, Strong Interactions of Single Atoms, Photons in Cavity QED, Phys. Scr. T T76, 127 (1998).

[17] H. Mabuchi and A. Doherty, Cavity Quantum Electrodynamics: Coherence in Context, Science 298, 1372 (2002).

[18] S. Yokoyama, R. Ukai, S.C. Armstrong, C. Sornphiphatphong, T. Kaji, S. Suzuki, J.-i. Yoshikawa, H. Yonezawa, N. C. Menicucci, and A. Furusawa, Ultra-Large-Scale Continuous-Variable Cluster States Multiplexed in the Time Domain, Nat. Photonics 7, 982 (2013).

[19] A. Wallraff, D. I. Schuster, A. Blais, L. Frunzio, R.-S. Huang, J. Majer, S. Kumar, S. M. Girvin, and R. J. Schoelkopf, Strong Coupling of a Single Photon to a 
Superconducting Qubit Using Circuit Quantum Electrodynamics, Nature (London) 431, 162 (2004).

[20] M. H. Devoret and R. J. Schoelkopf, Superconducting Circuits for Quantum Information: An Outlook, Science 339, 1169 (2013).

[21] R. Barends, J. Kelly, A. Megrant, A. Veitia, D. Sank, E. Jeffrey, T. C. White, J. Mutus, A. G. Fowler, B. Campbell et al., Superconducting Quantum Circuits at the Surface Code Threshold for Fault Tolerance, Nature (London) 508, 500 (2014).

[22] S. Haroche and J.-M. Raimond, Exploring the Quantum: Atoms, Cavities, and Photons (Oxford University, New York, 2006).

[23] M. Reagor, H. Paik, G. Catelani, L. Sun, C. Axline, E. Holland, I. M. Pop, N. A. Masluk, T. Brecht, L. Frunzio et al., Reaching $10 \mathrm{~ms}$ Single Photon Lifetimes for Superconducting Aluminum Cavities, Appl. Phys. Lett. 102, 192604 (2013).

[24] D. Leibfried, R. Blatt, C. Monroe, and D. Wineland, Quantum Dynamics of Single Trapped Ions, Rev. Mod. Phys. 75, 281 (2003).

[25] C. Monroe and J. Kim, Scaling the Ion Trap Quantum Processor, Science 339, 1164 (2013).

[26] C. Flühmann, T. L. Nguyen, M. Marinelli, V. Negnevitsky, K. Mehta, and J. P. Home, Encoding a Qubit in a TrappedIon Mechanical Oscillator, Nature (London) 566, 513 (2019).

[27] Z. Leghtas, G. Kirchmair, B. Vlastakis, R. J. Schoelkopf, M. H. Devoret, and M. Mirrahimi, Hardware-Efficient Autonomous Quantum Memory Protection, Phys. Rev. Lett. 111, 120501 (2013).

[28] M. Bergmann and P. van Loock, Quantum Error Correction against Photon Loss Using Multicomponent Cat States, Phys. Rev. A 94, 042332 (2016).

[29] N. Ofek, A. Petrenko, R. Heeres, P. Reinhold, Z. Leghtas, B. Vlastakis, Y. Liu, L. Frunzio, S. Girvin, L. Jiang et al., Extending the Lifetime of a Quantum Bit with Error Correction in Superconducting Circuits, Nature (London) 536, 441 (2016).

[30] L. Hu, Y. Ma, W. Cai, X. Mu, Y. Xu, W. Wang, Y. Wu, H. Wang, Y. P. Song, C. L. Zou, S. M. Girvin, L.-M. Duan, and L. Sun, Quantum Error Correction and Universal Gate Set Operation on a Binomial Bosonic Logical Qubit, Nat. Phys. 15, 503 (2019).

[31] S. Rosenblum, P. Reinhold, M. Mirrahimi, L. Jiang, L. Frunzio, and R. J. Schoelkopf, Fault-Tolerant Detection of a Quantum Error, Science 361, 266 (2018).

[32] A. S. Holevo, Probabilistic and Statistical Aspects of Quantum Theory (Springer Science, New York, 2011), Vol. 1.

[33] E. Knill, Quantum Computing with Realistically Noisy Devices, Nature (London) 434, 39 (2005).

[34] D. Bacon, Operator Quantum Error-Correcting Subsystems for Self-Correcting Quantum Memories, Phys. Rev. A 73, 012340 (2006).

[35] D. Kribs, R. Laflamme, and D. Poulin, Unified and Generalized Approach to Quantum Error Correction, Phys. Rev. Lett. 94, 180501 (2005).

[36] P. Aliferis and J. Preskill, Fault-Tolerant Quantum Computation against Biased Noise, Phys. Rev. A 78, 052331 (2008).
[37] P. Brooks and J. Preskill, Fault-Tolerant Quantum Computation with Asymmetric Bacon-Shor Codes, Phys. Rev. A 87, 032310 (2013).

[38] Whenever we talk about a code with $N$-fold rotation symmetry, we always implicitly assume that the code does not have any degree of rotation symmetry higher than $N$.

[39] It is also common to define nonorthogonal code words for cat codes, as in, e.g., Ref. [4], but we here follow the more recent convention from, e.g., Ref. [5].

[40] K. K. Sabapathy and C. Weedbrook, On States as Resource Units for Universal Quantum Computation with Photonic Architectures, Phys. Rev. A 97, 062315 (2018).

[41] S. S. Elder, C. S. Wang, P. Reinhold, C. T. Hann, K. S. Chou, B. J. Lester, S. Rosenblum, L. Frunzio, L. Jiang, and R.J. Schoelkopf, High-Fidelity Measurement of Qubits Encoded in Multilevel Superconducting Circuits, Phys. Rev. X 10, 011001 (2020).

[42] Note that the parity operator is $\hat{\Pi}_{2}^{0}-\hat{\Pi}_{2}^{1}$.

[43] This result can be checked using Eq. (6) and the fact that $\left(\hat{Z}_{N}\right)^{2 N}=\left(\hat{Z}_{N}\right)^{0}=\hat{I}$ and $\left(e^{i \pi \ell / N}\right)^{2 N}=\left(e^{i \pi \ell / N}\right)^{0}=1$.

[44] V. V. Albert, K. Noh, K. Duivenvoorden, D. J. Young, R. T. Brierley, P. Reinhold, C. Vuillot, L. Li, C. Shen, S. M. Girvin, B. M. Terhal, and L. Jiang, Performance and Structure of Single-Mode Bosonic Codes, Phys. Rev. A 97, 032346 (2018).

[45] Encoded logical gates are denoted by an overbar, e.g., $\bar{Z}$, while operators projected onto the code space are denoted, following Ref. [44], by the subscript "code."

[46] In Ref. [44] and related works, cat and binomial codes are described by a spacing parameter $S$, which is related to the code distance by $S=d_{n}-1 . S$ is the largest detectable number error.

[47] C. Bény and O. Oreshkov, General Conditions for Approximate Quantum Error Correction and Near-Optimal Recovery Channels, Phys. Rev. Lett. 104, 120501 (2010).

[48] V. V. Albert, S. O. Mundhada, A. Grimm, S. Touzard, M. H. Devoret, and L. Jiang, Pair-Cat Codes: Autonomous Error-Correction with low-Order Nonlinearity, Quantum Sci. Technol. 4, 035007 (2019).

[49] C. W. Helstrom, Quantum Detection, Estimation Theory, J. Stat. Phys. 1, 231 (1969).

[50] K. R. Motes, B. Q. Baragiola, A. Gilchrist, and N.C. Menicucci, Encoding Qubits into Oscillators with Atomic Ensembles and Squeezed Light, Phys. Rev. A 95, 053819 (2017).

[51] V.P. Belavkin, Optimal Distinction of Non-Orthogonal Quantum Signals, Radio Eng. Electron. Phys. 20, 39 (1975).

[52] V.P. Belavkin, Optimal Multiple Quantum Statistical Hypothesis Testing, Stochastics 1, 315 (1975).

[53] P. Hausladen and W. K. Wootters, A "Pretty Good" Measurement for Distinguishing Quantum States, J. Mod. Opt. 41, 2385 (1994).

[54] H. M. Wiseman and R. B. Killip, Adaptive SingleShot Phase Measurements: The Full Quantum Theory, Phys. Rev. A 57, 2169 (1998).

[55] B. L. Higgins, D. W. Berry, S. D. Bartlett, H. M. Wiseman, and G. J. Pryde, Entanglement-Free Heisenberg-Limited Phase Estimation, Nature (London) 450, 393 (2007). 
[56] A. A. Berni, T. Gehring, B. M. Nielsen, V. Händchen, M. G. Paris, and U. L. Andersen, Ab Initio QuantumEnhanced Optical Phase Estimation Using Real-Time Feedback Control, Nat. Photonics 9, 577 (2015).

[57] S. Daryanoosh, S. Slussarenko, D. W. Berry, H. M. Wiseman, and G. J. Pryde, Experimental Optical Phase Measurement Approaching the Exact Heisenberg Limit, Nat. Commun. 9, 4606 (2018).

[58] D. W. Berry and H. M. Wiseman, Optimal States and Almost Optimal Adaptive Measurements for Quantum Interferometry, Phys. Rev. Lett. 85, 5098 (2000).

[59] A. Holevo, Covariant Measurements and Imprimitivity Systems, in Quantum Probability and Applications to the Quantum Theory of Irreversible Processes (Springer, New York, 1984), pp. 153-172.

[60] H. M. Wiseman, D. W. Berry, S. D. Bartlett, B. L. Higgins, and G. J. Pryde, Adaptive Measurements in the Optical Quantum Information Laboratory, IEEE J. Sel. Top. Quantum Electron. 15, 1661 (2009).

[61] L. S. Martin, W. P. Livingston, S. Hacohen-Gourgy, H. M. Wiseman, and I. Siddiqi, Implementation of a Canonical Phase Measurement with Quantum Feedback, arXiv:1906.07274.

[62] We note that GKP codes defined for a quantum rotor, i.e., a particle moving on a circle described by angular position $\hat{\varphi}$ and angular momentum $\hat{N}$, have been studied previously $[3,63]$. However, the strictly non-negative spectrum of the number operator $\hat{n}$ implies that no unitary operator $e^{i \hat{\phi}}$ exists such that $\left[\hat{n}, e^{i \hat{\phi}}\right]=e^{i \hat{\phi}}[64]$. In this sense, the number and phase do not constitute a proper conjugate variable pair. See, e.g., Ref. [65] for a discussion on angle and phase operators in quantum mechanics and Refs. [64,66] for a proposal to define unitary and Hermitian phase operators as a limit of a sequence of regularized operators.

[63] P. Raynal, A. Kalev, J. Suzuki, and B.-G. Englert, Encoding Many Qubits in a Rotor, Phys. Rev. A 81, 052327 (2010).

[64] D. T. Pegg and S. M. Barnett, Phase Properties of the Quantized Single-Mode Electromagnetic Field, Phys. Rev. A 39, 1665 (1989).

[65] J.-M. Levy-Leblond, Who Is Afraid of Non-Hermitian Operators? A Quantum Description of Angle and Phase, Ann. Phys. (N.Y.) 101, 319 (1976).

[66] D. T. Pegg and S. M. Barnett, Unitary Phase Operator in Quantum Mechanics, Europhys. Lett. 6, 483 (1988).

[67] H. M. Wiseman, Adaptive Phase Measurements of Optical Modes: Going beyond the Marginal q Distribution, Phys. Rev. Lett. 75, 4587 (1995).

[68] K. Jacobs, A. N. Jordan, and E. K. Irish, Energy Measurements and Preparation of Canonical Phase States of a Nano-Mechanical Resonator, Europhys. Lett. 82, 18003 (2008).

[69] S. M. Barnett and D. T. Pegg, On the Hermitian Optical Phase Operator, J. Mod. Opt. 36, 7 (1989).

[70] Using the notation of Ref. [3], Pegg-Barnett codes as defined here with $s=p \times 2 N$ map onto shift-resistant qudit codes with $d=s, n=2, r_{1}=N$, and $r_{2}=p$.

[71] C. Flühmann, V. Negnevitsky, M. Marinelli, and J. P. Home, Sequential Modular Position and Momentum
Measurements of a Trapped Ion Mechanical Oscillator, Phys. Rev. X 8, 021001 (2018).

[72] Y. Zhang, X. Zhao, Z.-F. Zheng, L. Yu, Q.-P. Su, and C.-P. Yang, Universal Controlled-Phase Gate with CatState Qubits in Circuit QED, Phys. Rev. A 96, 052317 (2017).

[73] A. Kitaev, Protected Qubit Based on a Superconducting Current Mirror, arXiv:cond-mat/0609441.

[74] P. Webster, S. D. Bartlett, and D. Poulin, Reducing the Overhead for Quantum Computation when Noise Is Biased, Phys. Rev. A 92, 062309 (2015).

[75] C. Chamberland, P. Iyer, and D. Poulin, Fault-Tolerant Quantum Computing in the Pauli or Clifford Frame with Slow Error Diagnostics, Quantum 2, 43 (2018).

[76] Note that in this case, because $\bar{T}$ is not a Clifford gate, the correction for the - outcome $(i=1)$ is non-Pauli. The correction can be done by applying $\bar{X} \bar{S}$ for this outcome (using that $\bar{X} \bar{S} \bar{T} \bar{X}=-e^{-i \pi / 4} \bar{T}$, where the overall phase factor is unimportant). In fact, since $\bar{X} \bar{S}$ is equivalent to $\bar{S}$ up to a change of Pauli frame, we can simply apply $\bar{S}$ [77]. Alternatively, we can repeat the protocol until the desired outcome is achieved.

[77] B. M. Terhal, Quantum Error Correction for Quantum Memories, Rev. Mod. Phys. 87, 307 (2015).

[78] S. Bravyi and A. Kitaev, Universal Quantum Computation with Ideal Clifford Gates and Noisy Ancillas, Phys. Rev. A 71, 022316 (2005).

[79] An unfortunate clash in nomenclature has led to the state $|T\rangle$ that teleports the gate $\hat{T}$ being called an $H$-type (not $T$ type) magic state, because it is equivalent to a Hadamard eigenstate up to Clifford gates. Furthermore, in its original setting in Ref. [78], $\hat{T}$ is used to denote a different gate: that whose eigenstates are $T$-type magic states.

[80] B. C. Travaglione and G. J. Milburn, Preparing Encoded States in an Oscillator, Phys. Rev. A 66, 052322 (2002).

[81] B. M. Terhal and D. Weigand, Encoding a Qubit into a Cavity Mode in Circuit QED Using Phase Estimation, Phys. Rev. A 93, 012315 (2016).

[82] M. Eaton, R. Nehra, and O. Pfister, Gottesman-KitaevPreskill State Preparation by Photon Catalysis, arXiv: 1903.01925.

[83] Y. Shi, C. Chamberland, and A. W. Cross, Fault-Tolerant Preparation of Approximate GKP States, New J. Phys. 21, 093007 (2019).

[84] R. W. Heeres, B. Vlastakis, E. Holland, S. Krastanov, V. V. Albert, L. Frunzio, L. Jiang, and R. J. Schoelkopf, Cavity State Manipulation Using Photon-Number Selective Phase Gates, Phys. Rev. Lett. 115, 137002 (2015).

[85] S. Krastanov, V. V. Albert, C. Shen, C.-L. Zou, R. W. Heeres, B. Vlastakis, R. J. Schoelkopf, and L. Jiang, Universal Control of an Oscillator with Dispersive Coupling to a Qubit, Phys. Rev. A 92, 040303(R) (2015).

[86] A. Wünsche, Ordered Operator Expansions and Reconstruction from Ordered Moments, J. Opt. B 1, 264 (1999).

[87] Explicitly, $c_{k \ell}=(-1)^{k-\ell} s(k, \ell)$, where $s(k, \ell)$ is a Stirling number of the first kind [86].

[88] We can recognize that $c_{\ell}(\theta)=L(-\theta / 2 \pi, 0,-\ell)$ with $L(\lambda, \alpha, s)$ the Lerch zeta function. 
[89] S. Bravyi and R. König, Classification of Topologically Protected Gates for Local Stabilizer Codes, Phys. Rev. Lett. 110, 170503 (2013).

[90] F. Pastawski and B. Yoshida, Fault-Tolerant Logical Gates in Quantum Error-Correcting Codes, Phys. Rev. A 91, 012305 (2015).

[91] Of course, one also has to take into account that the type of error introduced by the gate is different from the initial error, and it is not clear cut whether a rotation error of magnitude $\pi k / N^{2}$ is better or worse than a number-shift error of magnitude $\hat{a}^{k}$ or $\left(\hat{a}^{\dagger}\right)^{k}$, even though they are both in some sense small when $k$ is small compared to $N / 2$. In general, which error is worse depends on the code's ability to deal with phase and number-shift errors.

[92] D. Gottesman, An Introduction to Quantum Error Correction and Fault-Tolerant Quantum Computation, in Proceedings of Symposia in Applied Mathematics, Quantum Information Science and Its Contributions to Mathematics, 2009 (American Mathematical Soc., Washington, DC, 2010), Vol. 68, pp. 13-58.

[93] B.C. Sanders, Superpositions of Distinct Phase States by a Nonlinear Evolution, Phys. Rev. A 45, 7746 (1992).

[94] E. Knill, Scalable Quantum Computing in the Presence of Large Detected-Error Rates, Phys. Rev. A 71, 042322 (2005).

[95] C. M. Dawson, H. L. Haselgrove, and M. A. Nielsen, Noise Thresholds for Optical Cluster-State Quantum Computation, Phys. Rev. A 73, 052306 (2006).

[96] For rotation codes that are not number-phase codes, i.e., codes with poor phase resolution $\Delta_{N}(\theta)$, it is possible that other measurements might be devised.

[97] J. C. J. Radtke, D. K. L. Oi, and J. Jeffers, Linear Quantum Optical Bare Raising Operator, J. Phys. B 50, 215501 (2017).

[98] M. A. Nielsen, A Simple Formula for the Average Gate Fidelity of a Quantum Dynamical Operation, Phys. Lett. A 303, 249 (2002).

[99] The average gate fidelity $F$ for a $d$-level system is related to the entanglement fidelity $F_{\text {ent }}$ as $F=\left(d F_{\text {ent }}+1\right) /(d+1)$ [98].

[100] Numerically, we implement phase measurements by discretizing the phase $\theta_{j}=\theta_{0}+j 2 \pi / J, j=1, \ldots, J$, and define POVMs $\hat{M}_{j}=\int_{\theta_{j}}^{\theta_{j+1}} d \phi|\phi, s\rangle\langle\phi, s|$, where $|\phi, s\rangle=$ $(1 / \sqrt{s}) \sum_{n=0}^{s-1} e^{i n \phi}|n\rangle$ is a truncated Pegg-Barnett phase state.

[101] M. Horodecki, P. Horodecki, and R. Horodecki, General Teleportation Channel, Singlet Fraction, and Quasidistillation, Phys. Rev. A 60, 1888 (1999).

[102] The inherent difficulty in performing one of the two syndrome measurements is a noticeable difference from GKP codes, where the two respective syndrome measurements for quadrature shifts are equally easy [3]. Fundamentally, the issue stems from the highly nonlinear number-translation "stabilizer" $\hat{\Sigma}_{N}$; cf. Eq. (18) and Fig. 12.

[103] GKP codes suffer a similar trade-off between resilience to position and momentum shifts [3].
[104] P. Aliferis and A. W. Cross, Subsystem Fault Tolerance with the Bacon-Shor Code, Phys. Rev. Lett. 98, 220502 (2007).

[105] A. W. Cross, D. P. Divincenzo, and B. M. Terhal, A Comparative Code Study for Quantum Fault Tolerance, Quantum Inf. Comput. 9, 541 (2009).

[106] J. Napp and J. Preskill, Optimal Bacon-Shor Codes, Quantum Inf. Comput. 13, 490 (2013).

[107] D. Gottesman, Quantum Fault Tolerance in Small Experiments, arXiv:1610.03507.

[108] Digitizing each bosonic mode measurement result as in Fig. 3 and performing a majority vote is unlikely to be the optimal scheme. Recent work on GKP codes shows that decoders that explicitly take advantage of continuous variable measurements have better performance $[12,13,15]$.

[109] S. Puri, L. St-Jean, J. A. Gross, A. Grimm, N. E. Frattini, P. S. Iyer, A. Krishna, S. Touzard, L. Jiang, A. Blais, S. T. Flammia, and S. M. Girvin, Bias-Preserving Gates with Stabilized Cat Qubits, arXiv:1905.00450.

[110] J. Guillaud and M. Mirrahimi, Repetition Cat Qubits for Fault-Tolerant Quantum Computation, Phys. Rev. X 9, 041053 (2019).

[111] Recently, it was shown that GKP codes can be decomposed into two subsystems - a logical qubit and gauge mode-according to their discrete translation symmetry [112], and a similar decomposition for rotation codes based on discrete rotation symmetry is likely. Note that the translation symmetry of any GKP code also implies twofold rotation symmetry (see Appendix C).

[112] G. Pantaleoni, B. Q. Baragiola, and N. C. Menicucci, Modular Bosonic Subsystem Codes, arXiv:1907.08210.

[113] S. E. Nigg, H. Paik, B. Vlastakis, G. Kirchmair, S. Shankar, L. Frunzio, M. H. Devoret, R. J. Schoelkopf, and S.M. Girvin, Black-Box Superconducting Circuit Quantization, Phys. Rev. Lett. 108, 240502 (2012).

[114] B. Q. Baragiola, G. Pantaleoni, R. N. Alexander, A. Karanjai, and N. C. Menicucci, All-Gaussian Universality and Fault Tolerance with the Gottesman-Kitaev-Preskill Code, Phys. Rev. Lett. 123, 200502 (2019).

[115] D. W. Berry, B. L. Higgins, S. D. Bartlett, M. W. Mitchell, G. J. Pryde, and H. M. Wiseman, How to Perform the Most Accurate Possible Phase Measurements, Phys. Rev. A 80, 052114 (2009).

[116] H.-K. Lau and M. B. Plenio, Universal Quantum Computing with Arbitrary Continuous-Variable Encoding, Phys. Rev. Lett. 117, 100501 (2016).

[117] Y. Y. Gao, B. J. Lester, K. S. Chou, L. Frunzio, M. H. Devoret, L. Jiang, S. M. Girvin, and R. J. Schoelkopf, Entanglement of Bosonic Modes through an Engineered Exchange Interaction, Nature (London) 566, 509 (2019).

[118] In Ref. [119] and related works, the coherent-state phases are given by $\omega^{k}=e^{i k \pi / d}$. In our notation, $d=N$ is the Fock-space code distance.

[119] L. Li, C.-L. Zou, V. V. Albert, S. Muralidharan, S. M. Girvin, and L. Jiang, Cat Codes with Optimal Decoherence Suppression for a Lossy Bosonic Channel, Phys. Rev. Lett. 119, 030502 (2017).

[120] Note that $\alpha$ and $r$ cannot both vanish, in which case the state is simply vacuum and two code words cannot be defined. 
[121] T. C. Ralph, A. Gilchrist, G. J. Milburn, W. J. Munro, and S. Glancy, Quantum Computation with Optical Coherent States, Phys. Rev. A 68, 042319 (2003).

[122] We use a different notation than previous references. Specifically, $N$ is denoted $S+1$ and $K$ is denoted $N+1$, where $S$ and $N$ are referred to as "spacing" and the "order," respectively, in Ref. [44].

[123] Note that $\hat{D}(\zeta)$ generates shifts in $q p$-phase space of magnitude $\sqrt{2}|\zeta|$. For example, $\hat{D}(\sqrt{\pi / 2})=e^{-i \sqrt{\pi} \hat{p}}$, which acts as a logical $\bar{X}$ for square-lattice GKP.

[124] Using standard quadrature operators $\hat{q}$ and $\hat{p}$ satisfying $[\hat{q}, \hat{p}]=i$, the lattice distances are expanded to $\sqrt{2} \alpha$ and $\sqrt{2} \beta$, and the area of the parallelogram in $q p$-phase space is $\pi$.

[125] Interestingly, $\bar{H} \bar{S}^{\dagger}=(\bar{S} \bar{H})^{\dagger}$, and $\bar{S} \bar{H}$ has $T$-type magic states as its eigenstates, where we use the original terminology from Ref. [78].

[126] H. J. Carmichael, S. Singh, R. Vyas, and P. R. Rice, Photoelectron Waiting Times and Atomic State Reduction in Resonance Fluorescence, Phys. Rev. A 39, 1200 (1989).

[127] H. M. Wiseman and G. J. Milburn, Quantum Theory of Field-Quadrature Measurements, Phys. Rev. A 47, 642 (1993). 